\title{
Polarizing Matter and Antimatter: A New Method
}

(The Study of a Repetitive Stern-Gerlach on Stored Polarized Protons and the

Spin-Splitter Experiment)

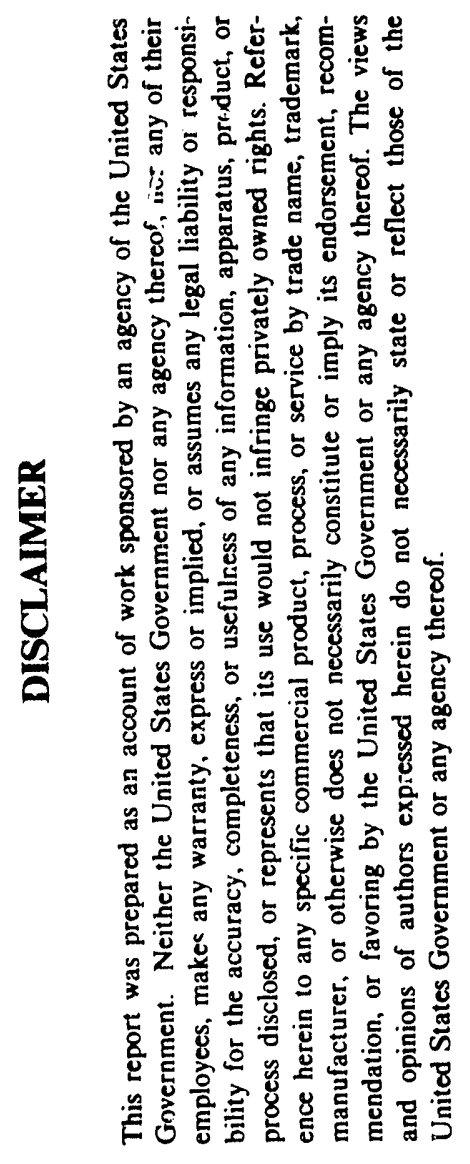

\author{
Yasar Onel (Principal Investigator) \\ Associate Professor of Physics \\ The University of Iowa \\ Iowa City, IA 52242
}

February 1992

DEPARTMENT OH ENERGY

AGREEMENT NO. DE-FG02-91ER40644 


\section{TABLE OF CONTENTS}

\section{Page}

ABSTRACT ................... . . iii

INTRODUCTION . . . . . . . . . . . . . . . . . . 1

THE PRINCIPAL CONCEPT . . . . . . . . . . . . . . . . . 2

Coherent Spin Motions . . . . . . . . . . . . . . . . . . . 3

Coherent Beatron Motions . . . . . . . . . . . . . . . . . 5

EXPERIMENTAL TOOLS AND PROCEDURES . . . . . . . . . . . 7

Snake Solenoid and Quadrupoles . . . . . . . . . . . . . . . . . . 9

The Polarimeter . . . . . . . . . . . . . . . . . . . . . . . . . 10

A. The Study of a Repetitive Stern-Gerlach Effect on Stored

Polarized Protons (CE-16) . . . . . . . . . . . . . . . . . . 12

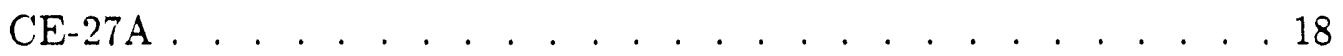

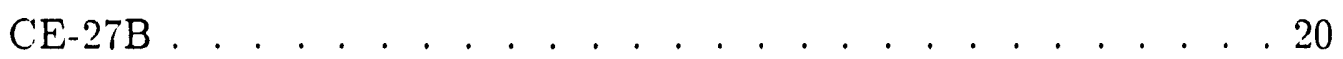

B. The Computer Simulations of Spin Stability . . . . . . . . . . . 36

Integration of the Spin-Splitter . . . . . . . . . . . . 36

Siberian Snake Experiment . . . . . . . . . . . . . . . . 38

Results . . . . . . . . . . . . . . . . . . .40

Summary . . . . . . . . . . . . . . . . . 45

C. Theoretical Studies . . . . . . . . . . . . . . . . . . . 59

REFERENCES . . . . . . . . . . . . . . . . 60

APPENDIX: PREPRINTS * hemoved 


\begin{abstract}
Several years ago a self-polarization effect for stored (anti-)protons and ions was investigated theoretically. The effect is based on the well-known Stern-Gerlach effect in gradient fields. The aim of the ongoing measurements at the Indiana University Cyclotron Facility (IUCF) is to verify experimentally the various assumptions on which this effect is based. The final goal is to demonstrate this new polarization effect. The proposed effect could be a powerful tool to produce polarized stored hadron beams both in the low-energy range and at SSC and LHC energies. In this progress report we will describe our progress in three parts: (A) Experimental work at IUCF Cooler Ring; (B) Our extensive computer simulations of the spin stability for the IUCF Cooler Ring; and (C) Theoretical studies.
\end{abstract}




\section{INTRODUCTION}

During the last few years a new polarization effect for stored protons or antiprotons was investigated theoretically $[1,2]$. The idea is the following: a storage ring consists of strong nonlinear fields, the quadrupoles. The nonlinear fields act on the spin of a particle because of the Stern-Gerlach effect causing the particles to be kicked by a very small amount. This small kick produces betatron oscillations. During the next revolution, the gradient of the quadrupole is (naturally) the same, but the spin direction can be altered. As a result, the strength and direction of the kick can be different from revolution to revolution. When this time-dependent kick follows a betatron oscillation, the kicks can add up over many revolutions, and a macroscopic oscillation can be excited which can be detected by an outside monitor. The principle is the same as in the one used for measuring the Q-value: small kicks add up over many revolutions and produce an oscillation with a significant plitude.

The aim of the ongoing measurements is to establish this effect. Although the above-mentioned concept is simple, straightforward, and somewhat of an analogy to well-established techniques, it differs in two points from those techniques.

First, the excitation strength is small and, therefore, a long time is needed to see the macroscopic effect. Since the strength of the kick is comparable to quantum-mechanical uncertainty, it was discussed at length whether the laws of quantum mechanics were imposing a principle limit on this effect. In addition, 
the long accumulation time raises questions concerning the stability of the field. Long-term drifts may smear out the effect.

Secondly, the driving force is related to the spin direction. The spin direction cannot be measured in a simple way, because the spin direction changes from revolution to revolution.

It was, therefore, amusing to learn that similar problems arise when gravitational wave detectors are designed $[6,7]$. Although different in the final goal, the underlying principles and limitations are nearly identical.

\section{THE PRINCIPAL CONCEPT}

When a proton passes a quadrupole, the acquired kick depends on the momentum of the particle:

$$
\eta^{\prime}=p_{t} / p,
$$

where $p=\beta \gamma m_{p} c, \gamma=\left(1-\beta^{2}\right)^{-1 / 2}$, and $m_{p}$ is the (anti-)proton mass. $p_{t}$ depends on the force $\mathrm{F}$ and the length of the interaction $\tau$. When $\mathrm{L}$ is the length of the interaction and $\beta c$ is the velocity, $\eta^{\prime}$ becomes:

$$
\eta^{\prime}=\frac{F L}{\beta^{2} \gamma m_{p} c^{2}} .
$$

The Stern-Gerlach force is $F=\nabla(\mu \cdot B)$ with $\mu=1,41 \cdot 10^{-10} J T^{-1}$. For low energies $\gamma \approx 1, \beta \approx 0.1$, a field of $20 \mathrm{Tm}^{-1}$ the kick is $9.4 \times 10^{-14} \mathrm{rad}$. In order to calculate the displacement in the machine, the kicks have to be multiplied with the square roots of the beta function. Assuming that the beta function (both at the point of observation and the point where the kick is applied) is $100 \mathrm{~m}$, the betatron 
amplitude is $\approx 10^{-8} \mathrm{~mm} .10^{8}$ revolutions are required to accumulate a betatron amplitude of $1 \mathrm{~mm}$.

Since most of the low-energy storage rings have revolution times between $10^{-6}$ and $10^{-7}$ seconds, a measurable amplitude should be accumulated within 10 to 100 seconds. During this time the spin motion and the betatron motion must be in phase. The resonance condition is:

$$
Q_{\text {spin }}=Q \pm n
$$

where $\mathrm{Q}$ is the $\mathrm{Q}$-value of the machine, $\mathrm{n}$ is any integer and $Q_{\text {spin }}$ is the number of spin precessions per revolution. In a machine without any spin manipulations, $Q_{\text {spin }}$ is $G \gamma$, where $G$ is the anomalous magnetic moment of the proton $(G=1.793)$. It should be mentioned that the superposition of Stern-Gerlach kicks on single charged particles is a well-established effect [3]. The difference in the single particle technique is only that an ensemble of particles and an ensemble of spins have to be considered. In order to avoid depolarization in such an ensemble, the spin motion of all particles must be (within certain limits) the same. The same is valid for the betatron motions. In addition, both motions must fulfill Eq. (1).

\section{Coherent Spin Motions}

Equation (1) defines the range of the spin tune. When $Q$ is an integer or a half-integer, the storage ring will not work. As a consequence, the spin tune $Q_{\text {spin }}$ must differ from an integer or half an integer. In other words, the spin direction has to change from revolution to revolution. Repetitions after each second revolution 
are not allowed. One of the aims of our experiments is to verify that such a spin oscillation is stable and does not lead to depolarization.

In principle, several different techniques can be applied to rotate the spin around the stable spin direction (the so-called $\overrightarrow{\mathbf{n}}$-axis). One is the Spin-Splitter idea [8], where the rotation is performed by a solenoid which rotates the spin by less than $180^{\circ}$. Figure 1 shows the whole ensemble which is more or less similar to a Siberian Snake with a solenoid. The skew quadrupoles have two functions: they compensate the influence of the solenoid on the particle trajectories and they act as Stern-Gerlach magnets. The magnets have opposite polarity, but due to the $180^{\circ}$ rotation of the solenoid, the Stern-Gerlach kicks add.

The other proposed technique is based on the application of time-dependent fields $[9,10]$ which manipulate the spin in a manner similar to the solenoid.

When we considered this experiment for the first time, an Ann Arbor-IUCF group [4] discussed a Siberian Snake experiment with a strong solenoid at IUCF at Bloomington, Indiana. IUCF is a proton storage ring with an electron cooler operating in the $100 \mathrm{MeV}$ to several $100 \mathrm{MeV}$ range. After the Siberian Snake group had finished part of their shifts, we asked for a few shifts to test some of our ideas.

The experiment was planned in three steps:

(a) The polarization is injected along the $\overrightarrow{\mathbf{n}}$-axis: the spin direction repeats from revolution to revolution (Fig. 2). The maximum beam current is accumulated. The polarization of the stored beam is measured. Since $Q_{\text {spin }}$ is an integer, an accumulation of Stern-Gerlach kicks is impossible. This measurement allows 
us to study the behavior of the polarimeter and efficiency of the accumulation process (stacking) in the presence of the Siberian Snake.

(b) The spin is injected $90^{\circ}$ relative to the $\overrightarrow{\mathbf{n}}$-axis. The spin is supposed to oscillate around the stable condition. Since the ideal Siberian Snake rotates the spin by $180^{\circ}$, the spin direction repeats after each second revolution (Fig. 3). A Stern-Gerlach accumulation can only be observed on a half-integer Q-value (which is not a stable condition). Nevertheless, this experiment allows us to study some technical details of the concept. Since the polarization must be the same after each second revolution, the polarimeter only measures a nonzero polarization when the input is gated with a subharmonic of the revolution frequency. The aim of this experiment is to study the stability of a polarization vector oscillating around the $\overrightarrow{\mathbf{n}}$-axis.

(c) The $180^{\circ}$ rotation of the Siberian Snake is changed into a $180-\Delta$ degree rotation. The spin does not repeat every second revolution (Fig. 4) and the resonance condition (1) can be fulfilled.

The progress on these experiments will be described in Section A.

\section{Coherent Betatron Motions}

A rather short time was spent getting more experimental information on Landau effects. Equation (1) assumes that the Q-value is the same for all particles. In reality the $\mathrm{Q}$-value depends on $\gamma$, the phase, and the amplitude of the betatron motion. The chromaticity (dependence of $Q$ on $\gamma$ ) is not compensated at IUCF. One would assume that with a single kick at the beginning, coherent oscillation is 
converted into an incoherent oscillation in a short time. This was verified experimentally [5].

But even with a fully compensated chromaticity, the Q-spread is big enough to reduce the excitation of the Stern-Gerlach kicks by Landau damping. A more detailed description of Landau damping can be found in Ref. [13]. Here only the basic elements are given.

The focusing strength of a nonideal quadrupole (quadrupole with sextupole, octupole, etc., components) depends on the amplitude of the particle trajectory. The amplitude means the distance between the center of the quadrupole and the actual trajectory. The amplitude changes from revolution to revolution (see Fig. 5) due to the fact that the $\mathrm{Q}$-value has to be a noninteger. As a result, the focusing strength is slightly different from revolution to revolution. Taking many revolutions into account, a distribution of Q-values around a central Q-value is obtained (Fig. 6).

Consider an ensemble of particles. Each particle has at each revolution a slightly different Q-value, even when the central Q-value for all particles is the same. When such an ensemble is excited by a monochromatic frequency from outside, only parts of the ensemble react with maximum amplitude. Others are only partly excited or not excited due to the fact that their oscillation frequency is different from the excitation frequency. The particles being fully excited change position with the partly excited and vice versa. The behavior of the ensemble can be described in a formal way as damping, the so-called Landau damping. In order to produce a significant amplitude, the excitation has to overcome the Landaudamping constant. 
During the last year, the effect of Landau damping on the Spin-Splitter experiment was discussed by several authors $[5,10,11]$. H. Kreiser [11] pointed out that this effect can reduce the achievable degree of polarization. Derbenev [10] argued that a coupling between the betatron oscillations can reduce the Landau-damping effects. This is well known and described in Ref. [13] in more detail. Here the effect is explained by two pendulums with slightly different frequencies.

When two pendulums are independent from each other and excited by one source, the sum of the amplitudes can be described by the above-mentioned Landaudamping picture. If the two pendulums are coupled by a (relatively weak) additional spring, the situation does not significantly change except for the fact that the whole assembly has an additional resonance frequency. This frequency is sharp. Derbenev argued that a quadrupole-type betatron oscillation is generated when particles with different spins get sepanated. The two parts of the quadrupole oscillations are coupled by strong electromagnetic forces when the particles are not relativistic. As a result, a new sharp frequency is generated and the Landau damping no longer reduces the separation.

\section{EXPERIMENTAL TOOLS AND PROCEDURES}

A floor plan of the Indiana University Cyclotron Facility (IUCF) is shown in Fig. 7. We injected into the Cooler Ring vertically polarized protons produced by an atomic beam type polarized ion source in terminal $\mathrm{A}$. The spin direction was flipped at the source approximately every 30 seconds. The $600 \mathrm{keV}$ proton beam from $_{1}$ terminal $\mathrm{A}$ was accelerated in the injector cyclotron and main cyclotron. The 
maximum energy of the second cyclotron is about $200 \mathrm{MeV}$. The initial polarization was measured usiıg the beamline 2 polarimeter located between the two cyclotrons. The spin rotator and polarization monitoring system [14] immediately following the main cyclotron is shown in Fig. 8. Experiments requiring horizontally injected beams used the $\theta$-solenoid to precess the spin from the vertical into the horizontal plane. The resulting polarization was measured with the beamline 3 and the beamline 5 polarimeters. The protons were deflected to beamline 9 which leads to the Cooler Ring.

The layout of the IUCF Cooler Ring is shown in Fig. 9. The Cooler Ring is a six-sided synchrotron storage ring with electron cooling, which can accelerate protons up to $500 \mathrm{MeV}$. The proton beam was injected into the ring using a fast, pulsed kicker magnet. The Siberian Snake was located in the S region. The beam was accelerated by a radiofrequency cavity in the A region. The vertical and radial components of the beam polarization were measured in the $\mathrm{G}$ region using a cylindrically syrametric polarimeter. The beam was electron cooled in the $\mathrm{C}$ region.

The experimental apparatus used in these measurements was provided by the IUCF, the Snake provided by BNL-Michigan-IUCF, and the RF solenoid provided by the University of Michigan groups. These groups have given precious support and advice for our runs.

In order to inject polarized protons into the Cooler Ring, two fast-pulsed kicker dipoles were constructed at the University of Michigan. We could inject the polarized beam into the Cooler with three different spin orientations at the 
Snake using the following schemes which to the current in $\theta$ and $\varphi$ solenoids can be arranged accordingly:

(a) $\hat{n}$ vector in IUCF at $a \gamma=2$

Direction of the polarization vector is longitudinal at the Snake. This is called the " $P_{l}$ scheme" as shown in Fig. 10.

(b) $\hat{n}$ vector in IUCF at $a \gamma=2$

Direction of the polarization vector is vertical at the Snake (for even turns, vertically outwards; for odd turns, vertically inwards). This is the " $P_{V}=$ vertical polarization" scheme as shown in Fig. 11.

(c) $\hat{n}$ vector in IUCF at $a \gamma=2$

Direction of the polarization vector is radial at the Snake (fur even turns, radially outwards; for odd turns, radially inwards). This is called the " $P_{r}$ scheme" as shown in Fig. 12.

Another scheme which we have not used so far is

$\hat{n}$ vector at $a \gamma=2.5$

which is called the " $P_{R}$ scheme" and shown in Fig. 13.

\section{Snake Solenoid and Quadrupoles}

The Michigan-IUCF Siberian Snake is located in the S region of the Cooler. The Snake consists of 2.2 T-m superconducting solenoid and eight quadrupole magnets. The Snake, as installed in the Cooler Ring, is shown in Fig. 14. In addition to precessing the proton spin by some desired amount, the solenoid also affects the beam orbit properties; the transverse phase space of the beam is rotated by 
about $30^{\circ}$ and the beam is focused by the solenoid. These effects are corrected by four quadrupolrs on each side of the superconducting solenoid. The four inner skew quadrupoles, which are mounted on rotating stands, rotate the beam to compensate for the spiral motion introduced by the main solenoid. The four outer quadrupoles compensate for the focusing effect of the solenoid and the rotated quadrupoles. With these compensating quadrupoles, the Snake should be optically transparent.

\section{The Polarimeter}

The polarimeter [15], which is located in the $\mathrm{G}$ region of the Cooler, was originally designed for use as a spectrometer in various nuclear physics experiments. The detector is azimuthally symmetric as shown in Fig. 15. The detector consists of two scintillator layers ( $F$ and $E$ ), which provide information on particle identification, and two pairs of orthogonal wire chambers ( $\mathrm{WC} 1$ and $\mathrm{WC} 2$ ), which were used in the single particle tracking mode to discriminate against nontarget related events. An event was defined by a coincidence between the $\mathrm{F}$ and the $\mathrm{E}$ detectors. The detector arrangement was adapted for use as a polarimeter by software segmenting the array into four quadrants: the up-down quadrants measured the radial component of the beam polarization and the left-right quadrants measured the vertical component of the polarization.

A $4.5 \mathrm{~mm}$ thick graphite slab is used as a so-called skimmer target. One edge of the target is exposed to the stored beam to intercept the tail of the transverse distribution. After injection the beam is moved towards the skimmer edge by changing the current in the trim coils of the corner dipoles upstream and downstream of 
the target region. The displacement of the beam is increased until a count rate is reached which is compatible with limits on data acquisition. Because of the $180^{\circ}$ phase advance between the two corner dipoles, the perturbation of the beam is localized to the target region. To keep the scattering rate constant, the beam position is controlled by a feedback circuit between the event rate and the trim coils of the corner dipoles of the Cooler. Thus, eventually all beam ions intercept the target.

The trace-back of the wire chambers is used to select events originating from the target. Since the detector is cylindrically symmetric, the horizontal and vertical polarization components can be extracted simultaneously. Figure 16(a) shows an on-line histogram of the energy distribution (sum of all detectors) for the reaction ${ }^{12} \mathrm{C}(\mathrm{p}, \mathrm{p}){ }^{12} \mathrm{C}$, where the elastic scattering peak is clearly dominant compared to the inelastic channel. Figure 16(b) shows the observed $\theta$ distribution for this polarimeter from the wire chamber ray-trace and Fig. 16(c) shows the on-line histogram of vertex distribution after an on-line cut was applied to eliminate background due to smallangle scattering. (These on-line histograms are from our October run.) 


\title{
A. The Study of a Repetitive Stern-Gerlach Effect on Stored Polarized Protons
}

\author{
CE-16: 10 Shifts
}

N. Akchurin, ${ }^{1}$ L. Badano, ${ }^{2}$ M. Conte,${ }^{2}$ R. Giacomich, ${ }^{3}$ M. Giorgi ${ }^{3}$

J. Hall, ${ }^{4}$ S. Hsueh, ${ }^{5}$ H. Kreiser, ${ }^{6}$ J. McPher'son, ${ }^{1}$ J. Nachtman, ${ }^{1}$

C. Newsom, ${ }^{1}$ Y. Onel,,${ }^{1 *}$ G. Pauletta, ${ }^{7}$ A. Penzo,${ }^{3 * *}$ A. Pisent,${ }^{8}$

M. Pusterla ${ }^{8}$ R. Rossmanith, ${ }^{9 * *}$ U. Strohbusch, ${ }^{6}$ and A. Yegneswaran ${ }^{9}$

Proposal P 88-104 was submitted to study at IUCF the feasibility of obtaining polarized (anti-)proton beams in storage rings by means of the Spin-Splitter, i.e., a device consisting of a pair of quadrupoles with opposite polarity [8], separated by a spin rotator (Snake). With the Spin-Splitter a macroscopic separation of opposite spin stites of circulating (anti-)protons could be obtained. The separation is caused by repetitive Stern-Gerlach kicks. The theoretical aspects of the method have been discussed extensively in the last years. Our proposal for a first experimental verification was reviewed by the IUCF Program Advisory Committee and was allocated 10 shifts for the first phase of measurements.

The measurements were supplemental to the program of the BNL-IndianaMichigan group on the basic properties of the Siberian Snake and were performed

1 University of Iowa, USA

2 Genova, Italy

3 Trieste, Italy

4 University of New Mexico, USA

${ }^{5}$ Fermilab, USA

${ }^{6}$ University of Hamburg, Germany

* Deputy Spokesman

7 Udine, Italy

** Spokesman

${ }^{8}$ Padova, Italy

9 CEBAF, USA 
during the two runs following the CE-15 data-taking periods. Then we had the opportinity to learn how to operate the Snake system and the polarimeter. A significant part of the time was spent tuning the accelerator and the polarimeter.

One of our major concerns was to achieve an appreciable spin separation with the Spin-Splister relating to the long-term stability of the polarization in the presence of a partially excited Siberian Snake.

To study this point, multiturn injection was used at IUCF for polarized beams and a polarized beam of high intensity was stored (up to $20 \mu \mathrm{A}$ ). As a result, we were able for the first time to measure the polarization of a single filling with sufficient accuracy. The beam was stored in the machine for relatively long times (10-20 minutes) before the polarization measurement was started.

The measurements have been performed at $107 \mathrm{MeV}(a \gamma=2)$ with the beam polarized in the equilibrium direction (longitudinal at the Snake). For these measurements, the Snake solenoid was operated both with a full field and a partial field $(25 \%)$. The results were very encouraging so that we could begin with the measurements of other crucial points. A first approach to manipulate and measure polarization outside the equilibrium spin direction was developed and partly tested. In addition, a first measurement of the damping of the coherent betatron motion was performed.

Experiment CE-16 had two scheduled runs with a total of 10 shifts. Before the first run (July 9th), we observed the CE-15 run. Based on this experience with the cooler, spin precessing solenoids (Snake and transfer-line rotators), and the 
polarimeter, we were able to start efficiently our experiment just after the CE-15 shifts. Our first aim was to study the long-term stability of the polarization.

We ran the cooler at $107 \mathrm{MeV}(a \gamma=2)$ and used multiturn injection to accumulate 1-2 $\mu A$. This intensity is high enough to measure the beam polarization with sufficient accuracy with the CE-01 polarimeter.

No special effort was dedicated during this first run to optimize the intensity or the lifetime of the stored beam, which turned out to be quite acceptable for our purposes (about $25 \mathrm{~min}$. with cooling).

The polarization of protons injected in the cooler was measured with the BL3 polarimeter and was always close to $75 \%$. We performed the following measurements:

(i) Vertical polarization injected, Snake off: $P_{v}=0.15, P_{r}=0.08$

(ii) Vertical polarization injected, Snake on (25\%): $P_{v}=0.09, P_{r}=-0.07$

(iii) Radial polarization injected, Snake on (25\%): $P_{v}=0.05, P_{r}=0.69$

All of these measurements were made immediately after injection. The accuracy is about \pm 0.05 for both components of the polarization. The solenoid was not compensated by skew quadrupoles during this run. Between the Snake and the polarimeter, the spin performs a precession of $60^{\circ}$. The result (iii) is then consistent with a full conservation of the polarization, since $P_{r}=0.75 \sin \left(60^{\circ}\right)=0.65$.

We then repeated measurement (iii), waiting up to 15 minutes after the filling time of 10 minutes:

(iv) Radial polarization injected, Srake on $(25 \%): P_{v}=-0.04, P_{r}=0.68$

(v) Vertical polarization injected, Snake on $(25 \%): P_{v}=-0.08, P_{r}=0.07$ 
(vi) Radial polarization injected, Snake on (25\%) during filling, then slowly switched off: $P_{v}=0.53, P_{r}=0.48$

(vii) Radial polarization injected, Snake off: $P_{v}=0.13, P_{r}=0.25$

From this initial set of measurements we can summarize:

- The polarization in the cooler is stable in the presence of a partial Snake over long times (10 min. accumulation +15 min. storage). This information is in agreement with the properties of partial Snakes found by CE-15 and shows that spin motion stability is at least as good as the beam lifetime.

- The Snake seems to have the largest effect during injection [compare results of (vi) to (vii)].

During the second run (15--18 August) at the same energy (107 MeV), work was concentrated on the following subjects:

(a) optimization of beam intensity and lifetime with the Snake at full strength,

(b) compensation of betatron coupling caused by the Snake with skew quadrupoles,

(c) polarization lifetime with spin along (and orthogonal to) the equilibrium ( $\overrightarrow{\mathbf{n}}$ axis) direction.

When the spins of particles are oscillating around the $\overrightarrow{\mathbf{n}}$-axis the polarization direction of the stored beam changes from revolution to revolution. In order to avoid depolarization, new particles can only be j..jected when the polarization direction of the stored beam and the injected beam is the same. Since the direction of the polarization of the injected beam does not change with time, a system to suppress injections when the two polarizations are not parallel was developed and tested. 
For a similar reason, the polarimeter has to be gated with the revolution frequency in order to measure stable polarization.

The "stroboscopic" injection and measurement gating systems were implemented and tested. They seemed to work rather well. Yet another effect destroyed the polarization. During the accumulation, the beam is debunched and the particles with different energies perform a different number of revolutions until the rebunching begins. Due to this effect, the number of passes of different particles through the Snake is different and the beam becomes depolarized. This effect could be compensated for by an additional RF-system which was not available yet.

During our shifts, the machine team optimized the machine by optimizing the $\mathrm{Q}$-values, correcting the closed orbit, and adjusting the e-cooling correction elements. We finally obtained a stored current of $20 \mu \mathrm{A}$. The beam lifetime was 20 minutes with cooling and 3.5 minutes without cooling.

\section{Summary}

The first runs of experiment CE-16 have verified the basic spin dynamics conditions for the operation of a Spin-Splitter. 


\title{
CE-27A
}

October 15 - October 18, 1991

\author{
N. Akchurin, ${ }^{1}$ L. Badano, ${ }^{2}$ A. Bravar,${ }^{1,3}$ M. Conte,${ }^{2}$ J. Hall, ${ }^{4}$ \\ J. McPherson, ${ }^{1}$ F. Olchowski, ${ }^{1}$ Y. Onel,,${ }^{1}$ A. Penzo, ${ }^{3}$ A. Pisent,${ }^{5}$ \\ M. Pusterla,${ }^{6}$ T. Rinckel ${ }^{7}$ and R. Rossmanith ${ }^{8}$
}

\section{$\underline{\text { Schedule }}$}

The aim of these first measurements is to check the conservation of the spin components transverse with respect to the stable solution $\mathbf{n}$. The total number of shifts for Experiment CE-27 ( A and B) was 22 shifts.

As a first trial to achieve this aim, we planned to inject the protons with their spins parallel to the $\overrightarrow{\mathbf{n}}$-axis at the Siberian Snake; then we thought of changing this alignment from longitudinal to transverse by making use of the RF-solenoid of Experiment CE-20.

\section{Procedure}

As a first test to be performed, we decided to measure the spin-flip induced by the RF-solenoid of Experiment CE-20. During the first shifts (from 15 October 9 p.m. to 16 October 8 a.m.), a resonance was found very quickly, was reproduced several times, but disappeared after a short interruption of the beam due to a drift

1 University of Iowa, USA

2 INFN and University of Genova, Italy

${ }^{3}$ INFN Trieste, Italy

${ }^{4}$ University of New Mexico, USA

${ }^{5}$ INFN-LNF, Legnaro (Padova), Italy

${ }^{6}$ INFN and University of Padova, Italy

${ }^{7}$ IUCF, Bloomington, Indiana, USA

${ }^{8}$ CEBAF, USA 
in the Snake current. The set of measurements were the horizontal polarization versus the RF-solenoid frequency with the following data:

$$
\begin{aligned}
& \mathrm{W}=103.95 \\
& I_{\text {snake }}=142.4 \mathrm{~A} \text { (full snake) } \\
& V_{\mathrm{RF}-\text { solenoid }}=14 K V_{p p}
\end{aligned}
$$

Pulse-duration: $\tau=50 \mathrm{msec}$

We found a $21 \%$-dip at $\nu_{\text {dip }}=2.2510 \mathrm{MHz}$ which soon drifted $50 \mathrm{~Hz}$ higher sitting around $39 \%, 53 \%, 51 \%, 58 \%$. This dip corresponds to a satellite on the left of the precessior frequency $1.5 \times f_{\text {rev }}=2.2550 \mathrm{MHz}$. By considering that we have $\frac{\nu_{\text {dip }}}{f_{\text {rev }}}=1.4974$, a possible explanation could be there was a small drift in the current of the Siberian Snake solenoid. In fact, such a precession frequency is produced by a rotation angle in the solenoid of $179.5^{\circ}$ instead of $180^{\circ}$.

In a successive shift (October 17 from 0:00 to 8:00 a.m.) with the set of parameters changed to

$$
\begin{aligned}
& \mathrm{W}=104.20 \\
& I_{\text {snake }}=144.2 \mathrm{~A} \\
& V_{\mathrm{RF} \text {-solenoid }}=14 K V_{p p} \text { (the same as before) } \\
& \text { Pulse-duration: } \tau=14 \mathrm{msec}
\end{aligned}
$$

we have observed the following: we have found a dip at $\nu_{\mathrm{dip}}=2.2630 \mathrm{MHz}$ with measured $P_{H}=49 \%$ at about 2:00 a.m. We repeated this measurement after roughly half an hour and found a net drift to $P_{H}=68 \%$. Almost 4 hours later, we found again at the same frecuency $P_{H}=54 \%$, which we immediately checked, drifted up to $73 \%$ and $77 \%$. 
Now $1.5 \times f_{\mathrm{rev}}=2.2572 \mathrm{MHz}$ (notice, however, that the ratio of the revolution frequencies of $103.95 \mathrm{MeV}$ and $104.20 \mathrm{MeV}$ is 1.001 ), we have $\frac{\nu_{\text {dip }}}{f_{\text {rev }}}=1.5039$. Repeating what was done before, we surmised that in this shift the precession angle in the solenoid could have been $180.7^{\circ}$.

As far as the other shifts are concerned, during the whole 16 October shifts, no depolarizing resonances were detected. In the shift (17 October 8:00 o.m. to 4:00 p.m.), we raised the voltage of the RF-solenoid up to $V_{p p}=25 \mathrm{KV}$ and tried with both short and long pulses. Short pulses $(10 \mathrm{msec})$ did not yield any significant results.

We then summarize by stating that we saw clear effects at $2.2510 \mathrm{MHz}$ and $2.2630 \mathrm{MHz}$. The central frequency in between these two values is $2.2570 \mathrm{MHz}$, which divided by 1.5 , yields a revolution frequency $f_{\mathrm{rev}}=1.504667 \mathrm{MHz}$ that nicely coincides with the RF display in the control room

$$
f_{\mathrm{rev}}=\frac{13.543045}{9} \mathrm{MHz}=1.504782 \mathrm{MHz}
$$

After a rather long shut-down, we had 2 hours left to continue our program. In order to show that a spin oscillation around the $\overrightarrow{\mathbf{n}}$-axis is stable, we injected a vertically polarized beam into the ring with the Snake on as before. We gated the polarimeter in such a way as to have data taken only after each second revolution. If a polarization is maintained transverse to the $\overrightarrow{\mathbf{n}}$-axis, the polarimeter will detect a vertical component $P_{V}$ of the polarization when the Snake is rotating the spin by $180^{\circ}$. Figure 17 shows the results of these measurements: i.e., $P_{V}$ versus the Snake current. By increasing the solenoid current, $P_{V}$ grows in the expected way and obviously decreases after a peak. 
Due to insufficient time, we could not measure the full curve on both sides and close to its maximum. Nevertheless, the clear correspondence with the theoretical forecasts is evident. For the first time, it is shown that non- $\overrightarrow{\mathbf{n}}$-axis polarization can survive under certain circumstances. A more detailed study was performed during the November run.

\section{$\underline{\mathrm{CE}-27 \mathrm{~B}}$}

November 13 - November 17

N. Akchurin,${ }^{1}$ L. Badano, ${ }^{2}$ A. Bravar,,${ }^{1,3}$ M. Conte,${ }^{2}$ J. McPherson,${ }^{1}$ F. Olchowski, ${ }^{1}$ Y. Onel, ${ }^{1}$ A. Penzo,${ }^{3}$ M. Pusterla,${ }^{4}$ T. Rinckel,${ }^{5}$ and R. Rossmanith ${ }^{6}$

\section{Summary}

The aim of these measurements was to check the conservation of both normal and side spin-components with respect to the stable solution $\overrightarrow{\mathbf{n}}$. As the first test, we injected the protons with vertical spins. Then we measured the polarization with full snake and with the polarimeter gated in such a way that it was picking up data every second revolution. As the second test, we injected protons with their spin lying on the horizontal plane and orthogonal to the $\overrightarrow{\mathbf{n}}$-axis at the Siberian Snake.

\footnotetext{
1 University of Iowa, USA

2 INFN and University of Genova, Italy

3 INFN Trieste, Italy

4 INFN and University of Padova, Italy

5 IUCF, Bloomington, Indiana, USA

6 CEBAF, USA
} 


\section{Introduction}

Our main goal was to further explore the trend in transverse polarization with respect to Snake current. The bunches were gated in order to pick-up data every second turn.

\section{Vertical Polarization}

We injected with the spin pointing in the vertical direction, i.e., with both solenoids BL3 and BL5 in the beam transfer line switched off. Since vertical polarization is maintained when particles revolve in a ring, spins are orthognnal (or normal) to the stable solution $\overrightarrow{\mathbf{n}}$ at the Siberian Snake solenoid. As this implies a continuous spin flipping, the polarimeter was gated in such a way as to take data after each second revolution. Proton energy, Snake-solenoid current, and time duration(s) are listed below.

$$
\begin{aligned}
& \mathrm{W}=106.3 \mathrm{MeV}(a \gamma=1.9959) \\
& I_{\text {snake }}=145.45 \mathrm{~A} \text { (full snake) } \\
& \text { spill time: } \tau_{\text {short }}=1 \mathrm{sec} \\
& \text { cycle time: } \tau_{\text {long }}=10 \mathrm{sec}
\end{aligned}
$$

The inelastic scattering background was quite large compared to tine elastic scattering peak. Nevertheless, an accurate selection of the elastic peak allowed us to obtain useful information as shown in Fig. 18, where $P_{V}$ vs. $I_{\text {snake }}$ is plotted.

We have also observed, quite randomly, some cases where the horizontal component $P_{H}$ was exhibiting values difierent from zero. This result could be interpreted as due to the nonperfect matching between the proton momentum and the solenoid current $I_{\text {snake. }}$. In fact, even though a small mismatch causes a spin ruation per 
revolution slightly different from $\pi$, this effect can add up over many turns giving rise to something detectable.

\section{Horizontal Polarization}

Protons were injected with their spins lying on the horizontal plane, having activated the solenoid BL5 in the beam transfer line. Due to the spin precession related to $a \gamma=2$, these spins are again orthogonal (sideways this time) to the stable solution $\overrightarrow{\mathbf{n}}$ at the Siberian Snake solenoid. Proton energy, snake, BL5 currents, and time duration(s) are listed below.

$$
\begin{aligned}
& \mathrm{W}=106.3 \mathrm{MeV}(a \gamma=1.9959) \text { as before } \\
& I_{\text {snake }}=145.45 \mathrm{~A} \text { (full snake) as before } \\
& I_{\mathrm{BL} 5}=34.0 \mathrm{~A} \\
& \text { spill time: } \tau_{1}=2.7 \mathrm{sec} \\
& \text { cycle time: } \tau_{2}=5 \mathrm{sec}
\end{aligned}
$$

The results obtained are shown in Figs. 19 and 20.

These are also very exciting and interesting results and the pattern of data in Fig. 20 (horizontally injected polarization beam) is very similar to the one in Fig. 18 (vertically injected polarization beam). We observe in both situations (Figs. 18 and 20) a main peak and two satellite peaks around the main peak (one on the right and one on the left) and only about $30 \%$ of the initial polarization survives. Figures 18 and 20 were produced with a "fine" scan of the current and the polarization gate was on. 


\section{$\underline{\text { Conclusions }}$}

There are significant indications that the transverse component survives across a depolarizing resonance energy with the Snake for a sufficiently long time. For the first time, it has been shown that a non- $\overrightarrow{\mathbf{n}}$-axis polarization can survive under certain circumstances. More details (that confirm and reinforce our conclusions) are expected from the off-line analysis. 


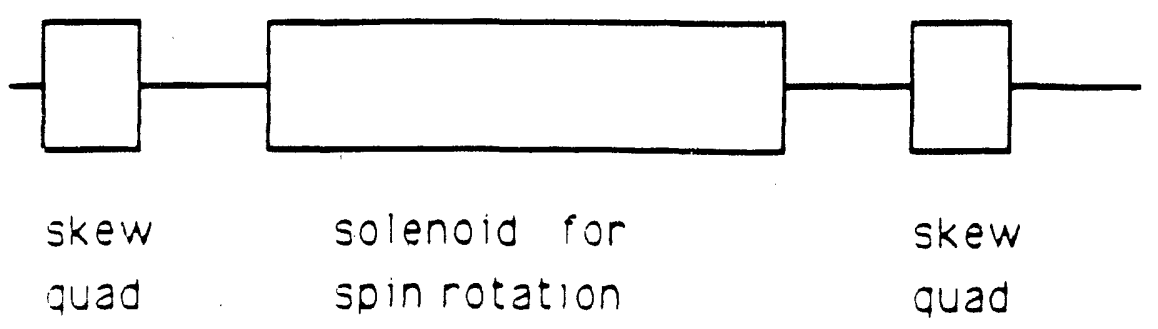

Figure 1. Magnet arrangement of the spin splitter.

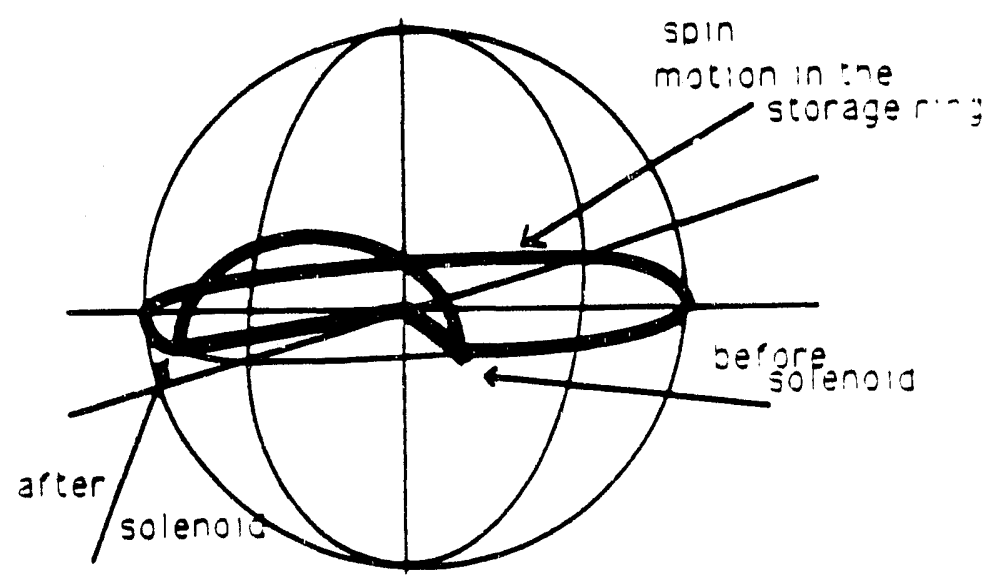

Figure 2. The stable spin direction in the presence of a 180 degree spin rotator.

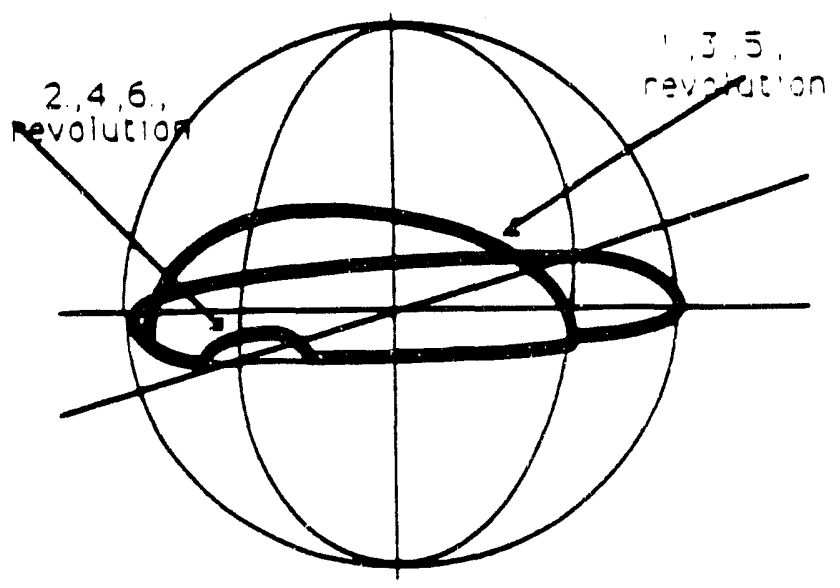

Figure 3. The injected polarization is oscillating around the stable spin direction. 


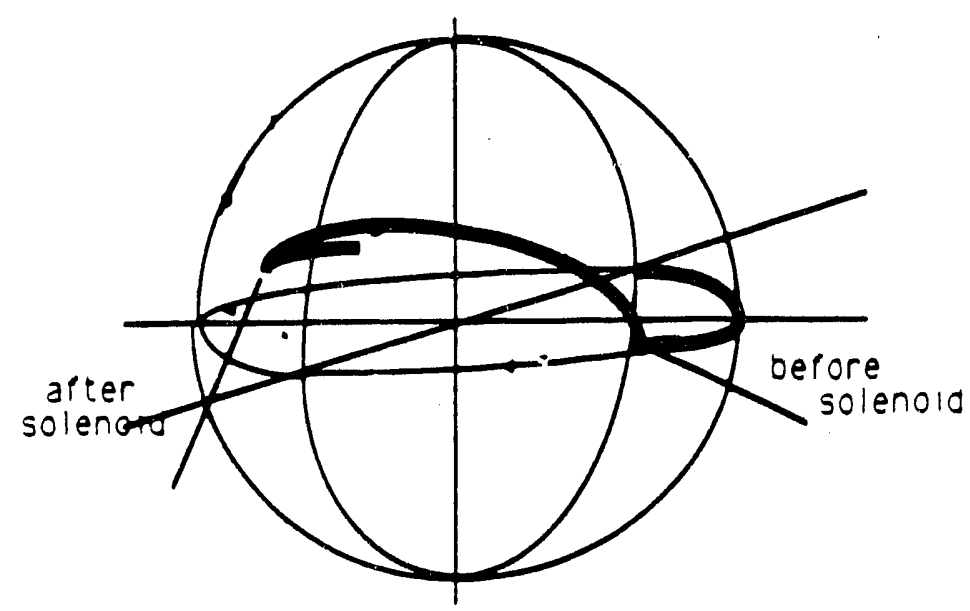

Figure 4. The solenoid does not rotate the spin by 180 degrees. The spin direction varies from revolution to revolution.

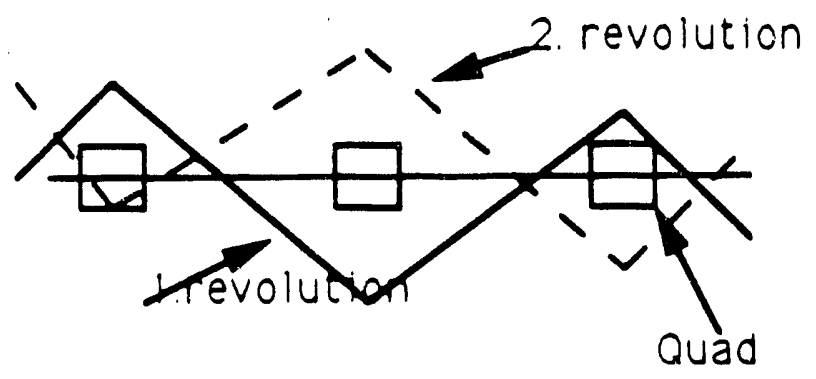

Figure 5. The betatron amplitude in a quad changes from revolution to revolution. As a result the nonlinearities in the quad change the Q-value from revolution to revolution.

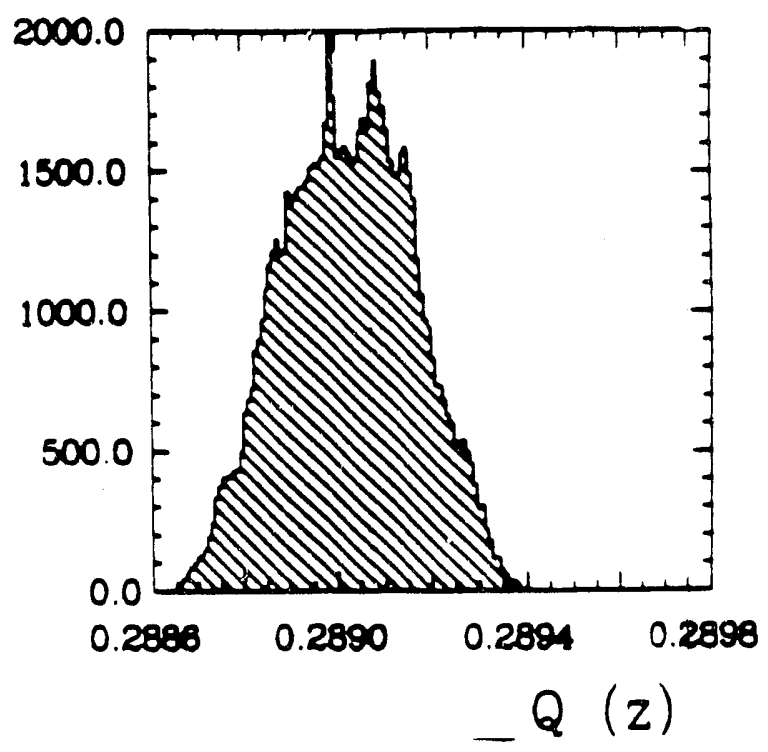

Figure 6. Calculated Q-value distribution (in relative 1 nits) for IUCF. 


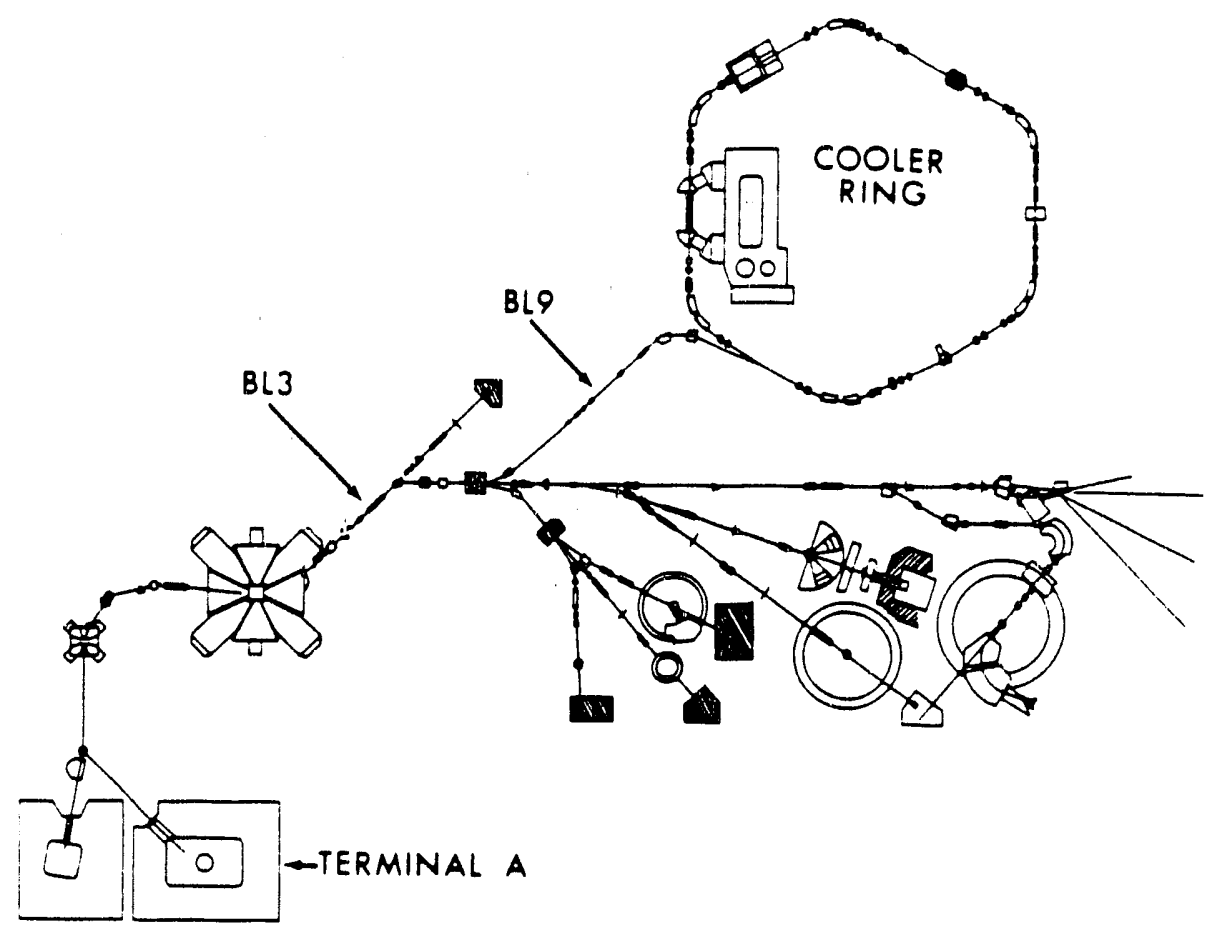

Figure 7. Layout of the Indiana University Cyclotron Facility. Polarized protons from the terminal $\mathrm{A}$ ion source were accelerated in the injector and main stage cyclotrons. They were then directed through beamline 3 , the switchyard, and beamline 9 to the Cooler Ring [16].

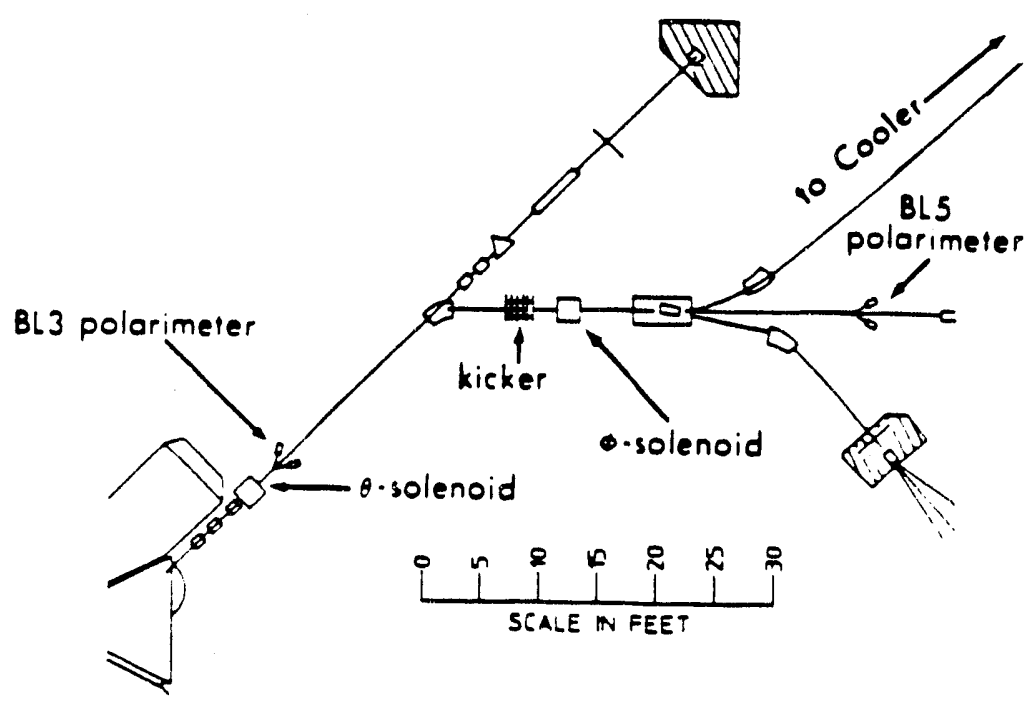

Figure 8. Spin rotator and monitoring system. The $\theta$ and $\phi$ solenoids are used to precess the nominally vertical polarization into the horizontal plane. $\vec{p}+d$ polarimeters in beamlines 3 and 5 are used to measure the injected polarization [16]. 


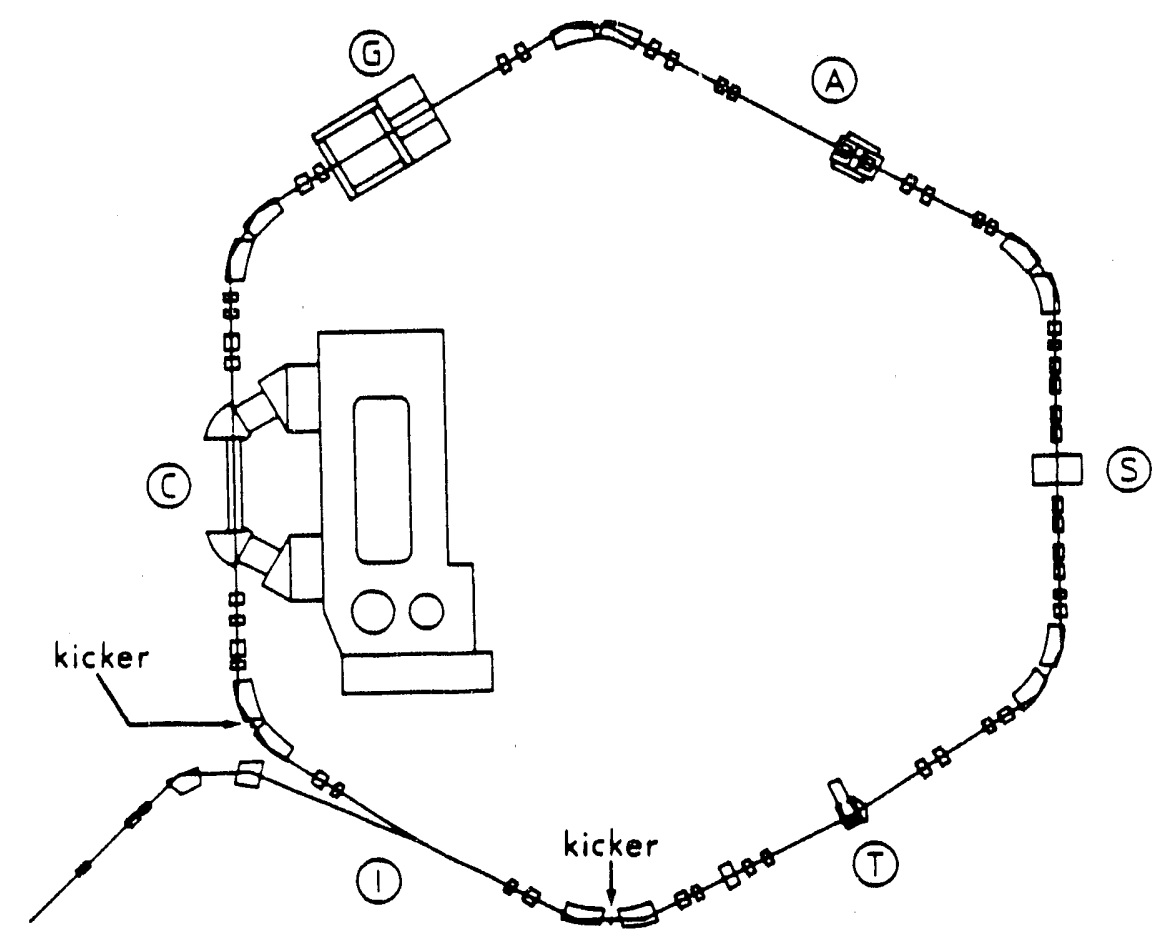

Figure 9. Layout of the IUCF Cooler Ring. Polarized protons were injected into the I region using a fast pulsed kicker dipole. The Siberian snake was located in the $\mathrm{S}$ region, the rf cavity was in the A region, the polarimeter was in the $\mathrm{G}$ region, and electron cooling took place in the $\mathrm{C}$ region [16].

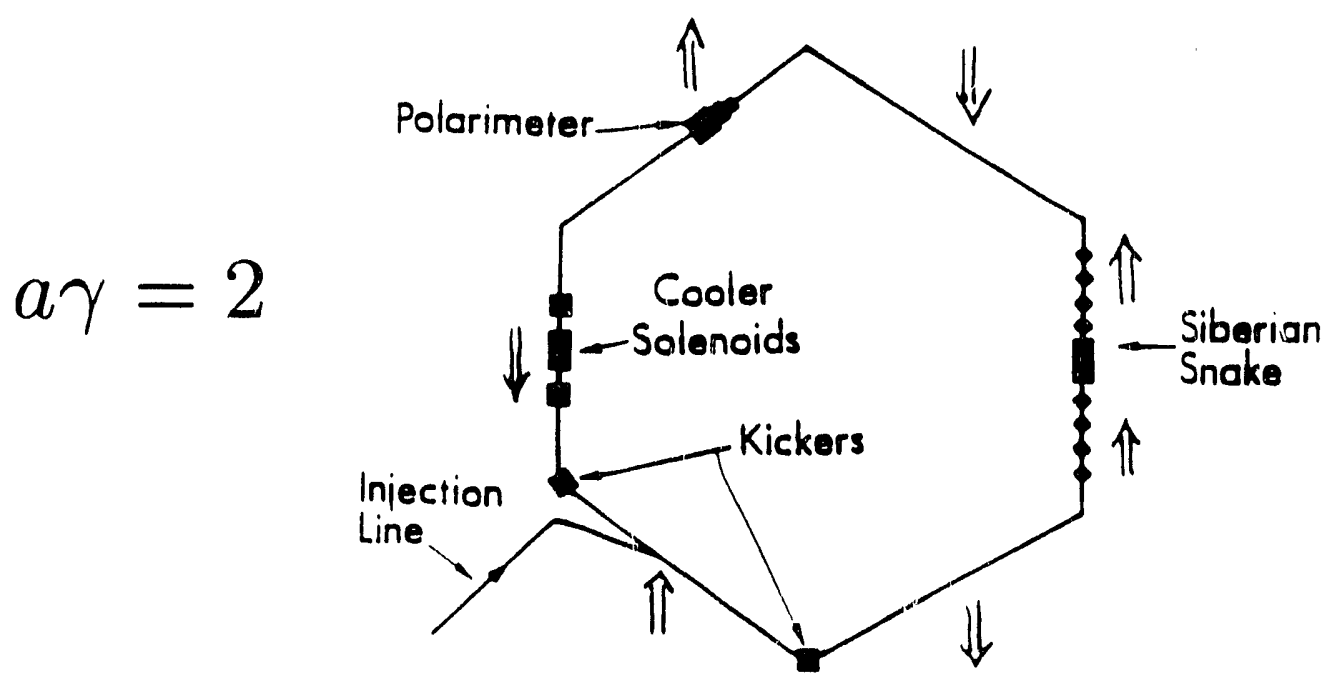

Figure 10. $P_{l}$ scheme. 


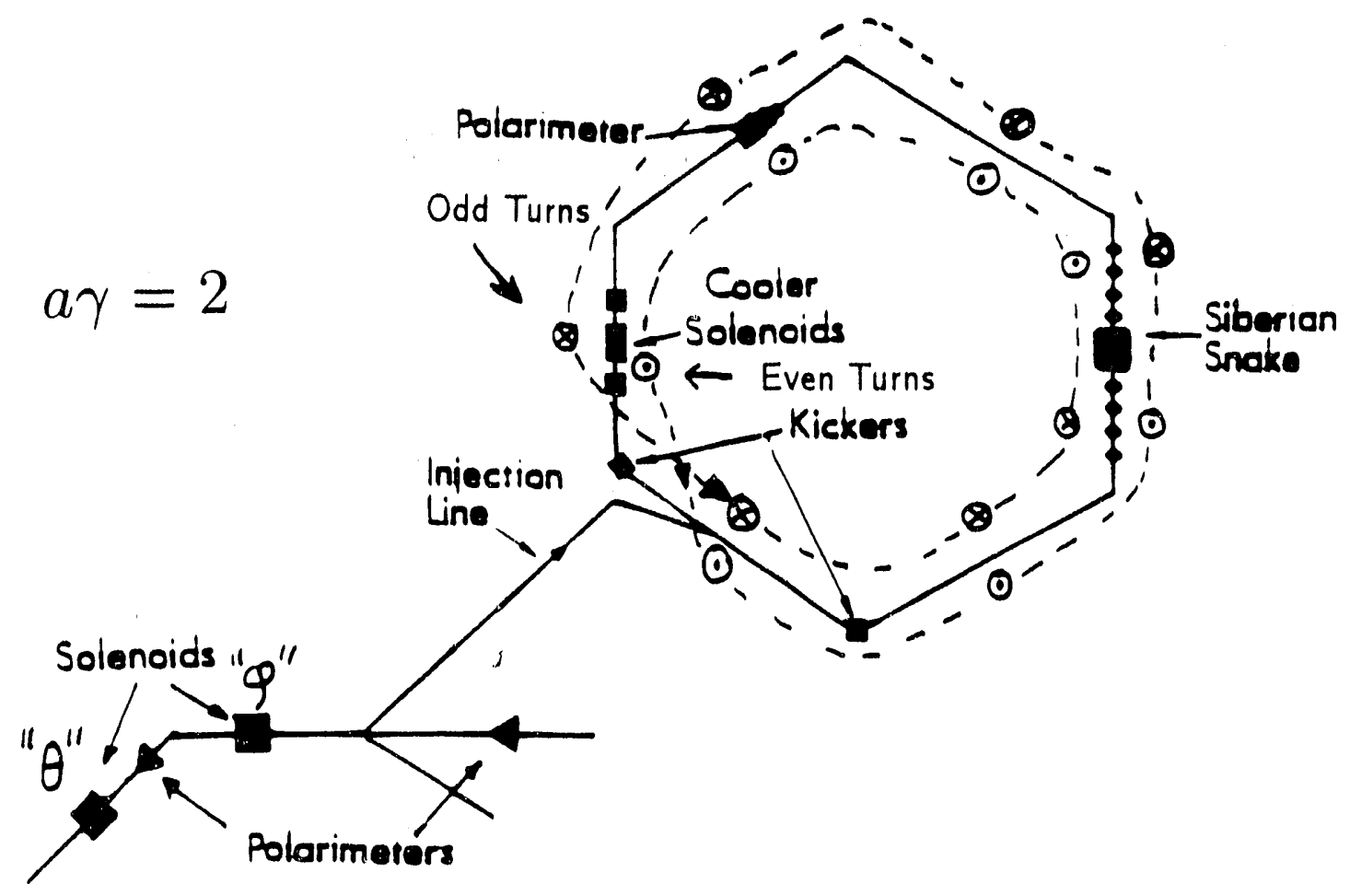

Figure 11. $P_{V}$ scheme.

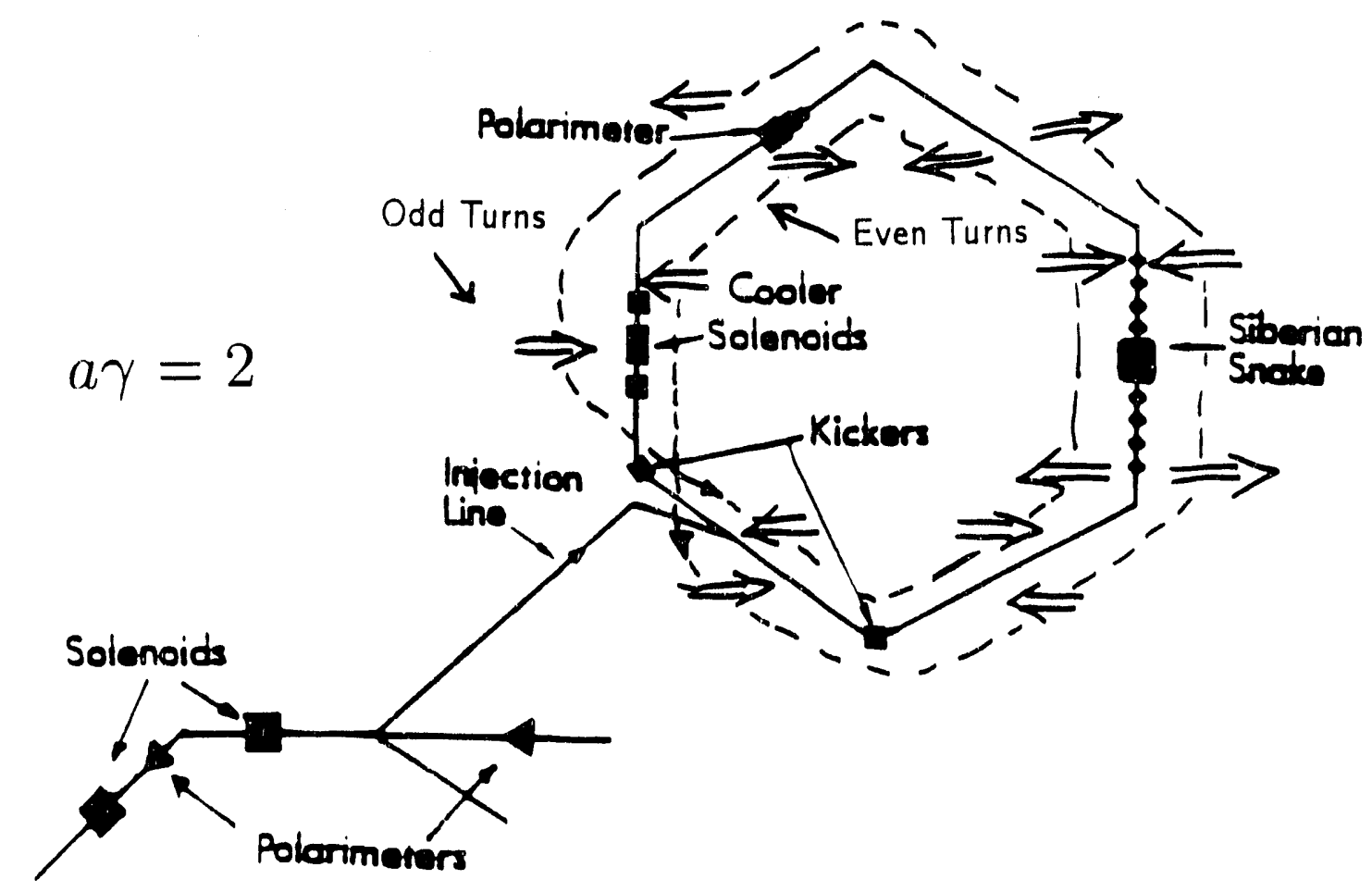

Figure 12. $P_{r}$ scheme. 


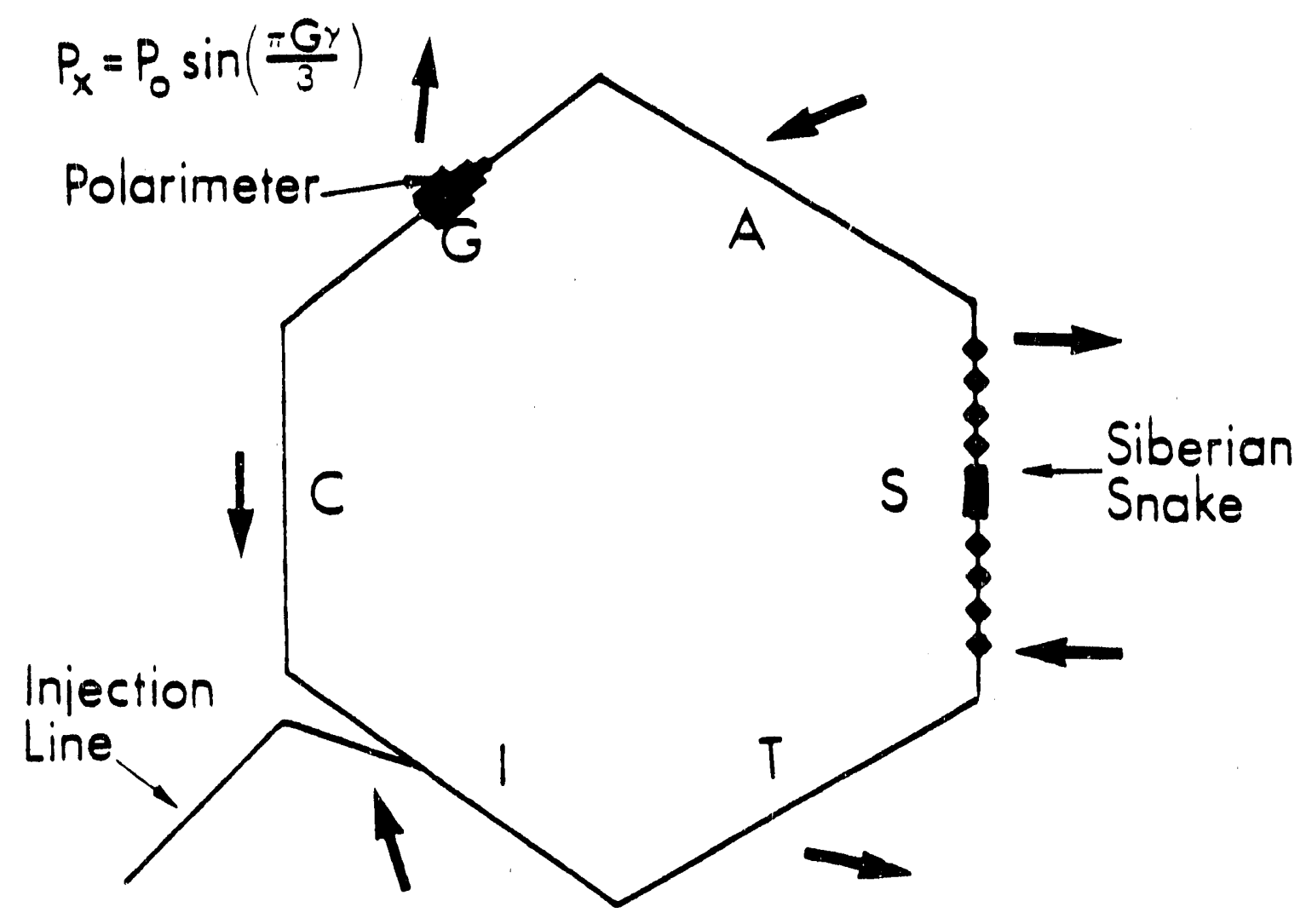

Figure 13. $\hat{n}$ vector at $a \gamma=2.5$

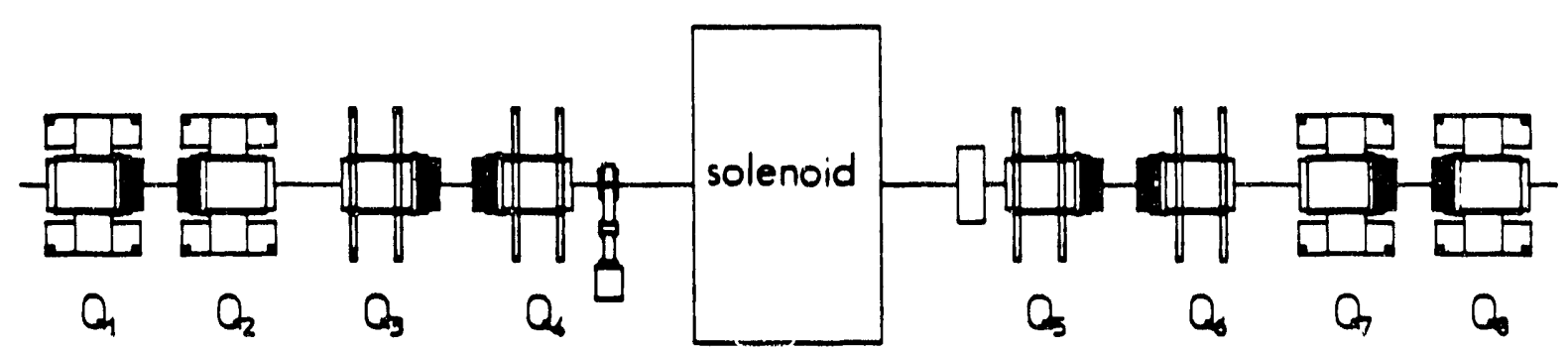

Figure 14. Layout of the Siberian snake. The Siberian snake consists of a superconducting solenoid, 4 skew quadrupoles $\left(\mathrm{Q}_{3-6}\right)$ and 4 normal quadrupoles $\left(Q_{1-2}, Q_{7-8}\right)[17]$. 


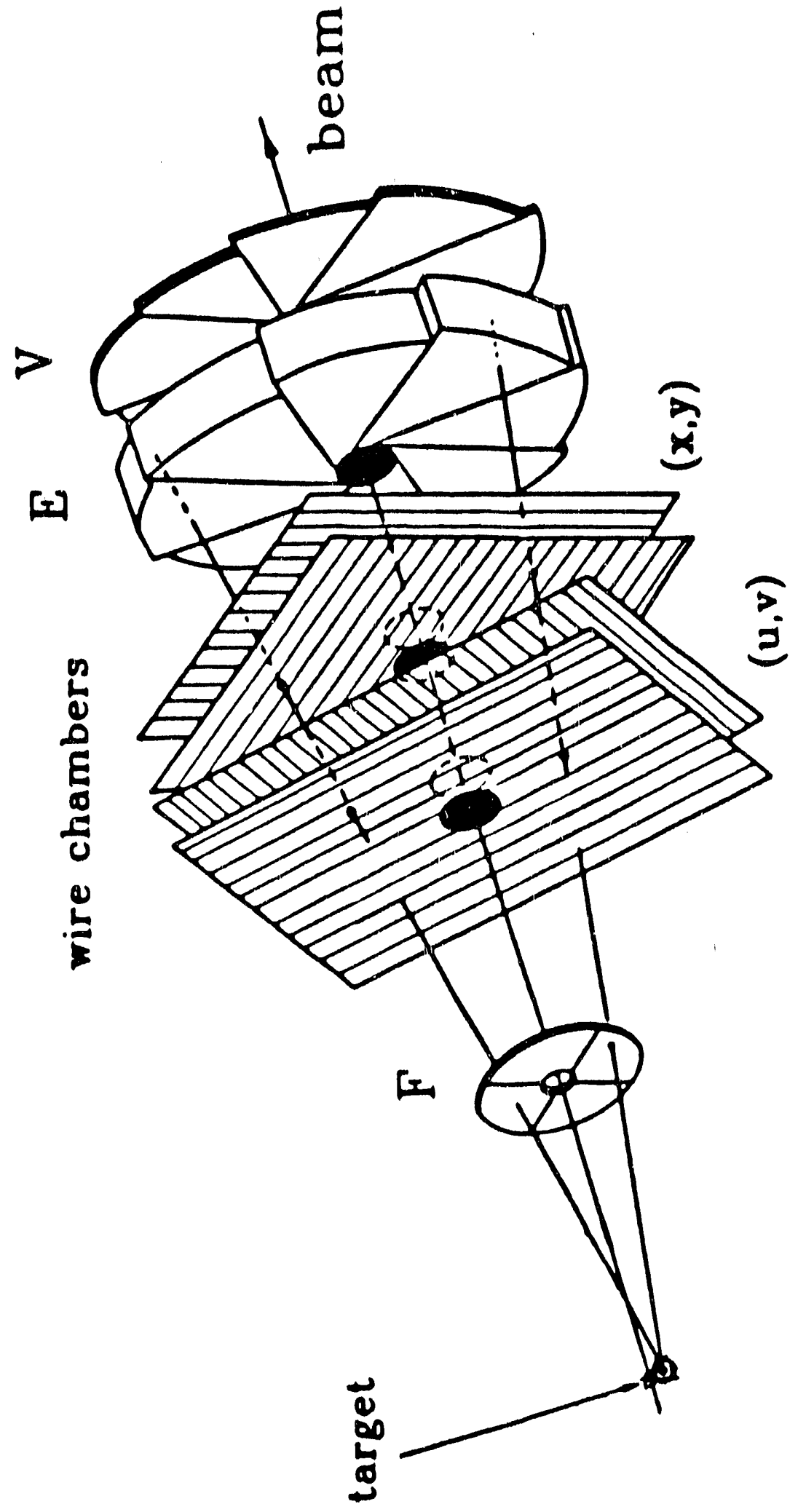

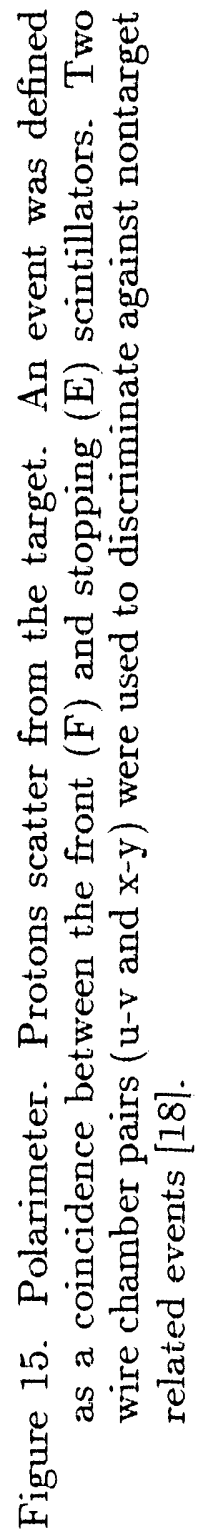



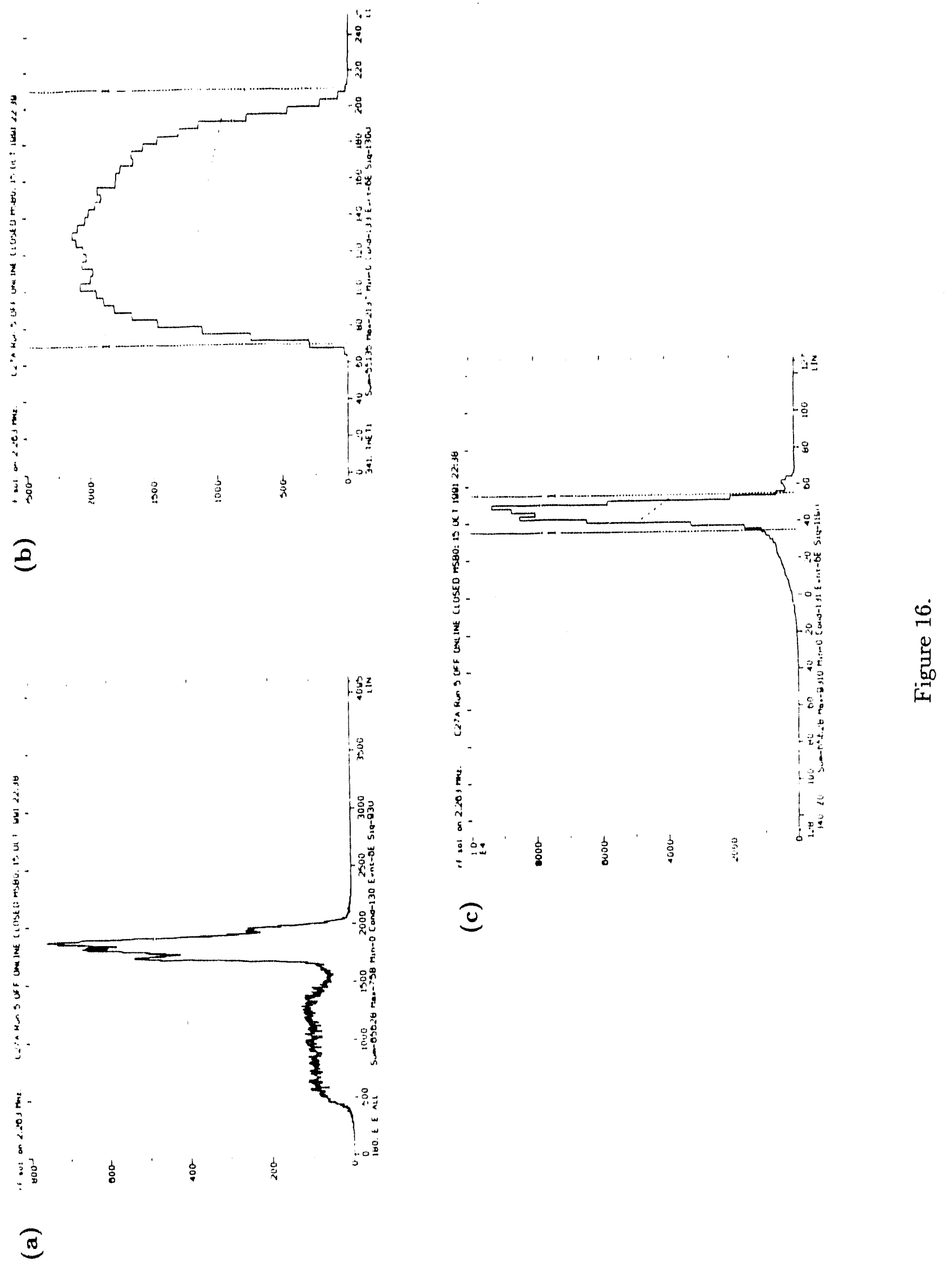

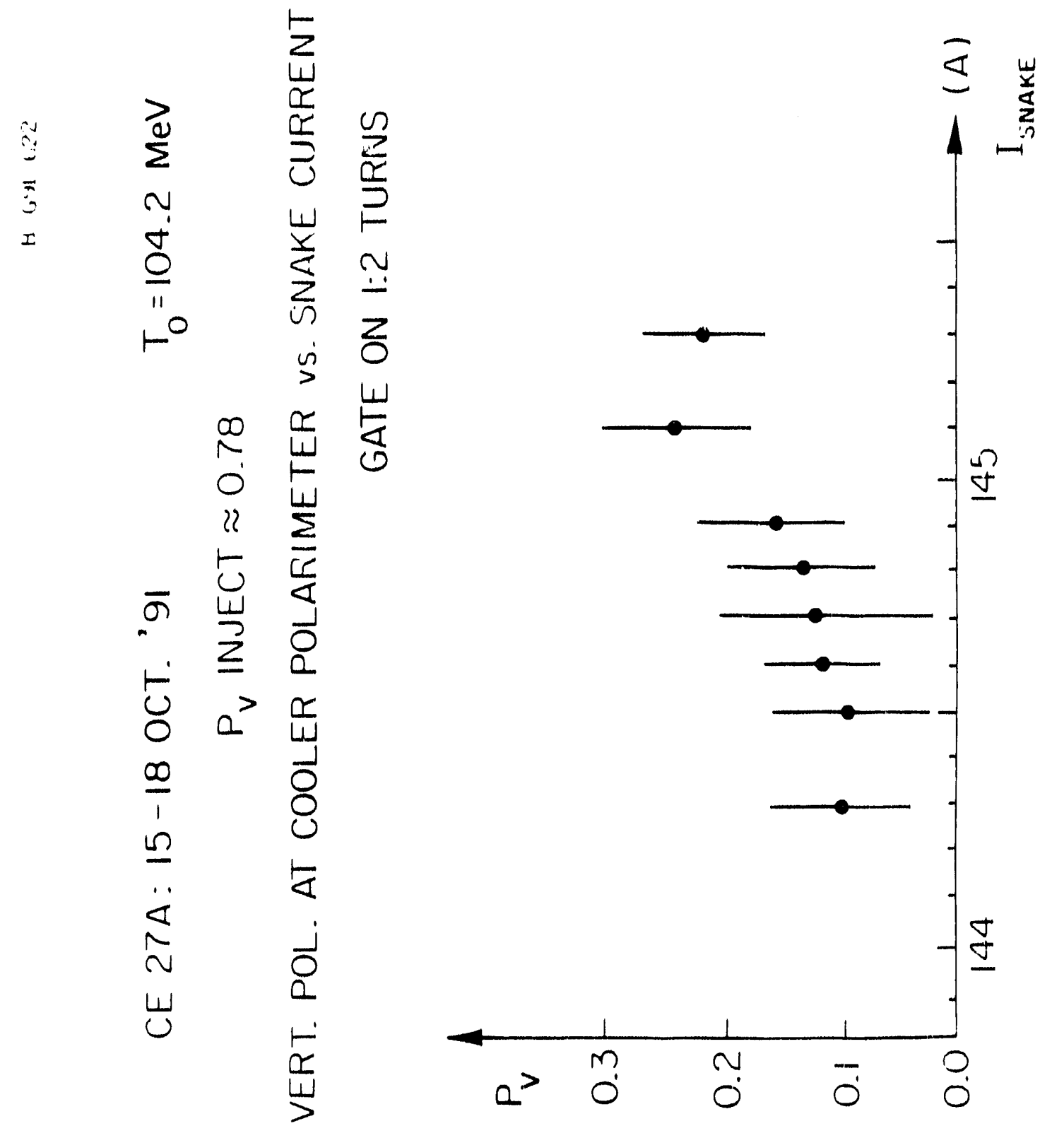

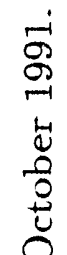

擎

青

명

号

侌 


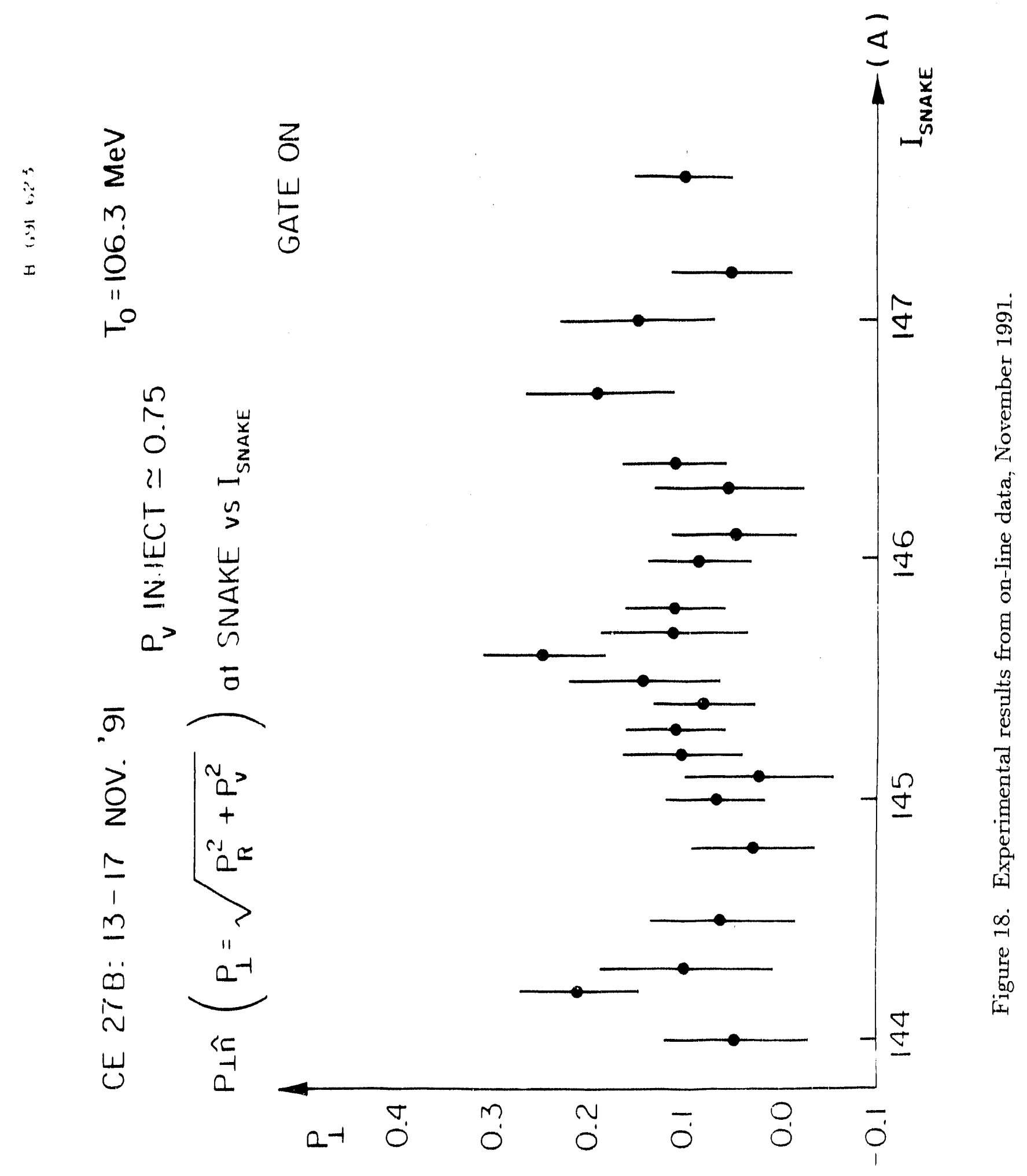




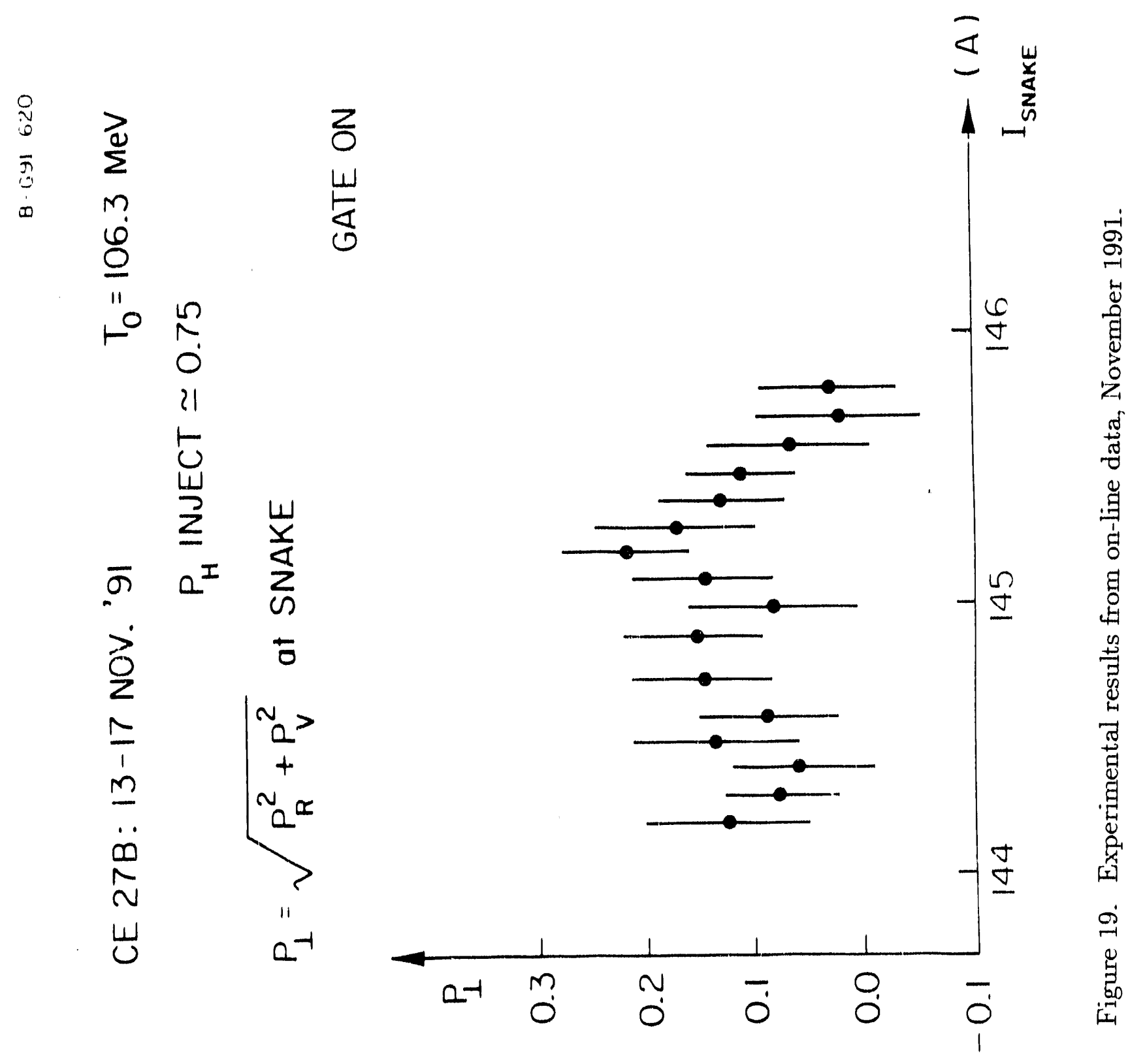




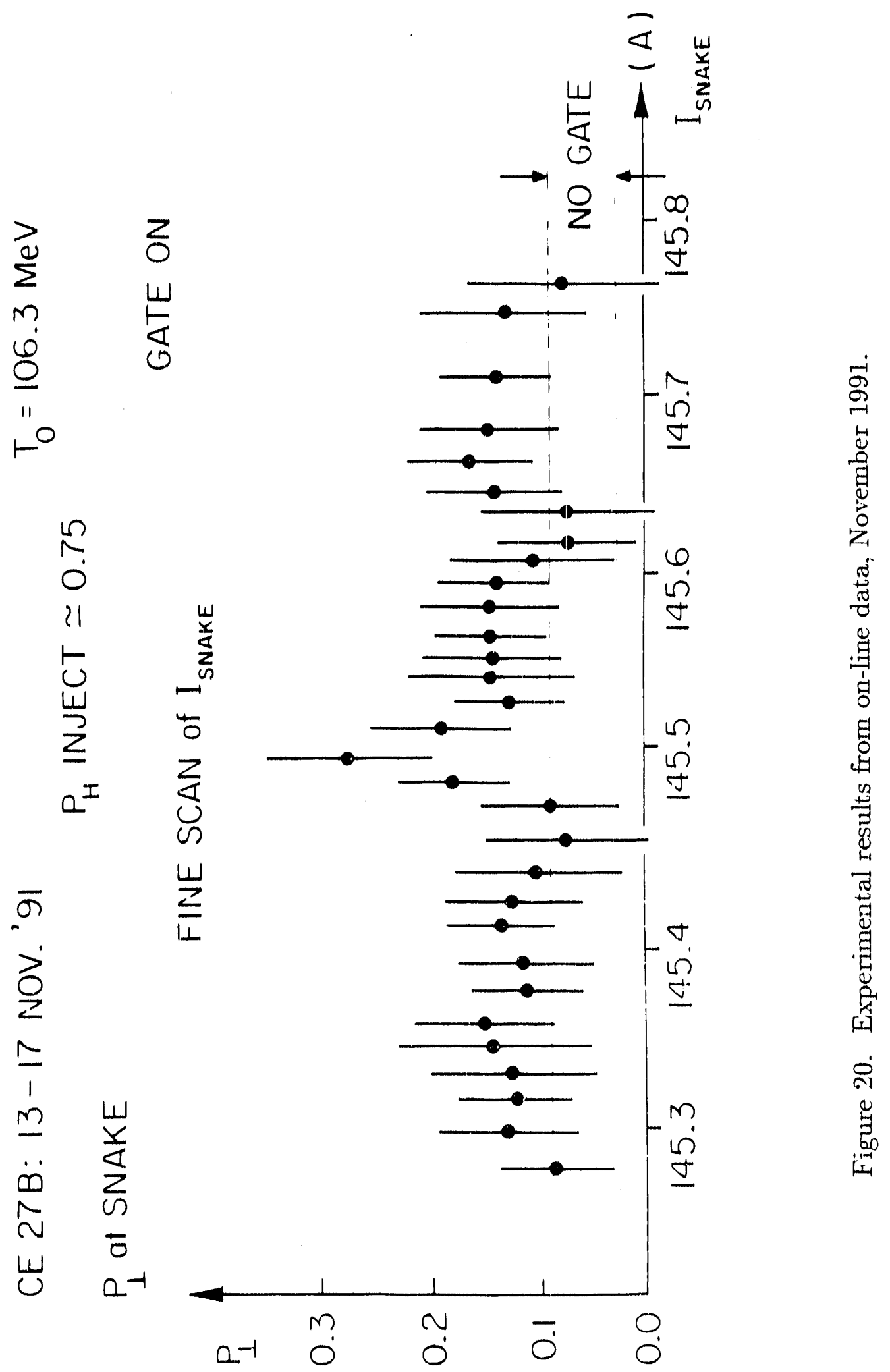




\section{B. Computer Simulations of Spin Stability}

H. Kreiser, University of Hamburg, Germany

Y. Onel, University of Iowa, USA

A. Penzo, University of Trieste, Italy

R. Rossmanith, CEBAF, USA

In addition to the measurements in Section A, optics calculations are performed. The aim of these calculations is to increase the net gradient in the quadrupoles adjacent to the Snake using special optics. These new optics allow us to enhance the Stern-Gerlach kicks.

\section{Integration of the Spin-Splitter}

Using a solenoid, the Spin-Splitter can be used only at low-energy storage rings. In order to rotate the spin by $\pi$, the following integral field as a function of energy is required:

$$
\int B d l[\mathrm{Tm}]=3.752 \cdot p \quad[\mathrm{GeV} / \mathrm{c}] .
$$

Superconductive solenoids are available up to 12 Tesla. The solenoid should be short (the length should not exceed $2 \mathrm{~m}$ ) and, therefore, the maximum momentum cannot exceed $7 \mathrm{GeV} / \mathrm{c}$.

In the following, the integration of the Spin-Splitter into the proton storage ring at the Indiana University Cyclotron Facility (IUCF) (circumference about $86 \mathrm{~m}$, $\left.P_{\max }=1.08 \mathrm{GeV} / \mathrm{c}\right)$ is discussed.

The Spin-Splitter (see Fig. 21) consists of a solenoid and two skew quads on each side. 
The field strength of the solenoid is determined by the amount of the required spin rotation. Field strength and distance between the quadrupoles are variable. Since there is not much space available, the distances should be as short as possible.

The unit has fulfilled two conditions: the decoupling of the trajectories and the field strength of the skew quads as high as possible. The calculations showed the following: optical decoupling requires that the strength of the quads depends on the solenoid field, and varying the distance enables the field strength to change the quads within a certain limit.

In order to solve the problem, a fit program using the program MINUIT [19] was developed. This program changes for a given solenoid strength and given distances between all magnets the strength of the skew quads until the coupling in the $4 \times 4$ matrix of the whole unit is very small. The decoupled Spin-Splitter was integrated into a ring and the ring optics matched to accommodate the unit.

The end fields of the solenoid act like strong focusing quads. Due to the short focusing length, the first compensating quadrupole must have a small distance to the solenoid. The compensation must work in both planes. The solutions shown in the following are a compromise between the short length of the whole unit, the highest possible field strength of the skew quads, and good matching to the rest of the ring.

\section{Integration in IUCF, Possibility \#1}

In IUCF additional matching quads could be used. The solenoid $(L=0.32 \mathrm{~m})$ used has a spin rotation of $180^{\circ}$ or $270^{\circ}$ an integrated field strength of: 


$$
\begin{aligned}
& \int B\left(\phi=180^{\circ}\right) d l=1.74 \mathrm{Tm}, \quad p=463 \mathrm{MeV} / \mathrm{c} \Rightarrow B_{\text {Sol }}=5.44 \mathrm{~T} \\
& \int B\left(\phi=270^{\circ}\right) d l=2.61 \mathrm{Tm}, \quad p=463 \mathrm{MeV} / \mathrm{c} \Rightarrow B_{\text {Sol }}=8.16 \mathrm{~T}
\end{aligned}
$$

With optimized skew quads an integral field gradient of $11.6 \mathrm{~T}$ can be achieved leading to a separation time of

$$
2 \delta \mathrm{x} \sim 0.4 \mathrm{~mm} / \mathrm{h}
$$

The Q-values change for a spin rotation of $270^{\circ}$ :

$$
\begin{aligned}
& \mathrm{Q}_{\mathrm{x}_{0}}=4.2646 \\
& \mathrm{Q}_{\mathrm{z}_{0}}=5.289
\end{aligned} \Rightarrow \begin{aligned}
& \mathrm{Q}_{\mathrm{x}}=4.299 \\
& \mathrm{Q}_{\mathrm{z}}=7.75
\end{aligned}
$$

By using an additional matching magnet $\left(L_{q}=0.27 \mathrm{~m}\right)$, the total length is $4.62 \mathrm{~m}$. Figure 22 shows the $\beta$-function (Fig. 23 the dispersion). The IUCF ring before (Fig 24) and after (Fig 25) the integration. After the integration, the $\beta$-function is slightly changed. Within the Spin-Splitter, a vertical dispersion is generated.

\section{Siberian Snake Experiment}

In Ref. [4] the first results with a Siberian Snake were described. Since this device can also be used as a Spin-Splitter unit, it was included in the simulations. The magnets have the following values:

$$
\begin{aligned}
& \text { Solenoid } L=0.69 \mathrm{~m} \rightarrow \int B\left(\phi=180^{\circ}\right) d l=1.74 \mathrm{Tm} \text {, } \\
& p=463 \mathrm{MeV} / \mathrm{c} \Rightarrow B_{\text {Sol }}=2.52 \mathrm{~T} \\
& \text { Solenoid } L=0.69 \mathrm{~m} \rightarrow \int B\left(\phi=216^{\circ}\right) d l=2.09 \mathrm{Tm} \\
& p=463 \mathrm{MeV} / \mathrm{c} \Rightarrow B_{\text {Sol }}=3.03 \mathrm{~T} \text {. }
\end{aligned}
$$


The skew quads $(L=0.345 \mathrm{~m}$ ) have an integral field gradient of $9.98 \mathrm{~m}$. The quads are rotated by $28^{\circ}$ and $12.2^{\circ}$ (different from the above-mentioned $45^{\circ}$ ). As a consequence, the separation is reduced by about $1 / 3$. This results in a separation time (for a rotation angle of $216^{\circ}$ ) of

$$
2 \delta \mathrm{x} \sim 0.18 \mathrm{~mm} / \mathrm{h}
$$

The $\mathrm{Q}$-values are changed by a spin rotation of $180^{\circ}$ by

$$
\begin{aligned}
& \mathrm{Q}_{\mathrm{x}_{0}}=4.2646 \\
& \mathrm{Q}_{\mathrm{z}_{0}}=5.289
\end{aligned} \Rightarrow \begin{aligned}
& \mathrm{Q}_{\mathrm{x}}=4.288 \\
& \mathrm{Q}_{\mathrm{z}}=6.259
\end{aligned}
$$

and by a spin ratation of $216^{\circ}$

$$
\begin{aligned}
& \mathrm{Q}_{\mathrm{x}_{0}}=4.2646 \\
& \mathrm{Q}_{z_{0}}=5.289
\end{aligned} \Rightarrow \begin{aligned}
& \mathrm{Q}_{\mathrm{x}}=6.23 \\
& \mathrm{Q}_{\mathrm{z}}=6.094 .
\end{aligned}
$$

The total length including four matching quads is $5.91 \mathrm{~m}$. Figure 24 shows the $\beta$-function (Fig. 25 the dispersion) of the Siberian Snake unit after integrating it into the IUCF ring.

\section{$\underline{\text { Results }}$}

The following working points were chosen for the calculations:

$$
\nu_{\mathrm{s}}=\mathrm{Q}_{\mathrm{z}} \pm \mathrm{m}, \quad \mathrm{a} \gamma=\mathrm{n}, \quad \mathrm{n} \in \mathrm{IN}
$$

Due to this working point, the optics change slightly. 
The simulations were performed for several different $Q$-values. For comparison, the simulations were also performed outside the resonance. The following cases were calculated

$$
\begin{array}{ll}
\text { 1. } & \nu_{s}=\mathrm{Q}_{\mathrm{z}}=0.75 \\
\text { 2. } & \nu_{s}=\mathrm{Q}_{\mathrm{z}}=0.7 \\
\text { 3. } & \nu_{s}=\mathrm{Q}_{\mathrm{z}}=0.7+\delta .
\end{array}
$$

In order to test the program PROTEUS, some test calculations were performed. The effect of the Siberian Snake on a polarized beam was especially tested. The results were compared with the measurements [4].

With the help of a Fourier analysis of the particle trajectories, the degree of orbit decoupling of a Siberian Snake can be determined. Since the Stern-Gerlach kick in the program was artificially increased, the Fourier technique allows us to analyze if this increase effects the simulation results.

With a spin tune of $\mathrm{a} \gamma=2$, the position of the spin is reproduced after each revolution. Perturbations lead to a fast depolarization.

The simulation shows this behavior. Figure 26 shows the $\mathrm{x}$ and $\mathrm{z}$ projection of the polarization after 1000 revolutions. The beam is injected with $P(z)=-1$. The figure shows the slow spread of the spins. It is expected that after more revolutions the spin directions are equally distributed in space. The perturbations are also clearly visible in the Q-distribution and the Fourier analysis. Figure 28 shows the distribution of the Q-values. This distribution is clearly broader compared to the unperturbed case. The Fourier spectrum shows the additional resonances (see Fig. 29). 


\section{Original Optics of the IUCF Ring}

For test purposes, the behavior of the beam with the original optic was investigated and compared with the measurements.

\section{IUCF without Sextupoles}

The IUCF ring has a length of $86 \mathrm{~m}$. The nominal $\mathrm{Q}_{z}$ value is $4.2446, \mathrm{Q}_{\mathrm{x}}=$ 5.289 .

Figure 30 shows the simulated $\mathrm{Q}_{\mathrm{z}}$ distribution. The average values correspond to the linear predictions and the Q-values oscillate around the calculated average value (see Fig. 35). Figure 36 shows a Fourier analysis of the particle oscillations.

\section{$\underline{\text { IUCF with Sextupoles }}$}

Sextupoles generate harmonics and coupled oscillations. Figure 37 shows the frequency spectrum for $Q_{x}$ and $Q_{z}$ with a strong main resonance and a few side resonances. This results in a broader distribution (compare Fig. 34 and Fig. 36). The oscillation of the $\mathrm{Q}$-values remains the same. The amplitudes are larger (especially in $\mathrm{x}$ ). This can be seen in Fig. 39 (compare with Fig. 35). Additional side bands generated by sextupoles cause disturbances in the particle trajectories. The vertical phase ellipse shows small structural changes (Fig. 36). The chromaticity correction by sextupoles causes position depending focusing and, therefore, changes of the trajectories especially in the $\mathrm{x}$-plane (see Fig. 41). In this figure the difference between the length of the calculated closed orbit and the actual particle trajectory is shown. The distribution has a maximum close to 0.04 . 


\section{Polarized Beam in z Outside a $Q_{z}-\nu_{s}$ Resonance}

The energy of the ring was changed to a $\gamma=2.5$. At that energy no depolarization is expected. The $Q_{\mathbf{z}}$ value equals the spin tune:

$$
\mathrm{Q}_{\mathrm{z}}=\nu_{\mathrm{s}}=0.47 \pm \mathrm{n}
$$

Figure 40 shows the spin in the direction of the $\overrightarrow{\mathbf{n}}$-axis. Compared with Fig. 39, the spin remains stable. There is no component in the direction of the $\overrightarrow{\mathbf{n}}$-axis. The polarization of the injected beam was $P(z)=-1$. In this case a partial snake is strong enough to stabilize the polarization.

\section{IUCF Optics with Integrated Siberian Snake}

Two different Siberian Snake configurations were calculated for IUCF. Both configurations were compared by applying the Fourier analysis technique in order to find the strength of the coupling and instabilities.

Siberian Snake, Possibility \#1. The layout was described on page 38 . The following ring parameters were used:

$$
\begin{aligned}
& \text { 1. } \mathrm{Q}_{\mathrm{z}}=\nu_{\mathrm{s}}=0.75 \\
& \text { 2. } \mathrm{Q}_{\mathrm{z}}=\nu_{\mathrm{s}}=0.7 .
\end{aligned}
$$

Case \#1 coincides with an octupole resonance. In general, this is not a stable working point. Since the simulation neglects octupole terms, the beam remains stable during the simulation. However, in the phase diagram, the well-known discontinuities appear. 
Case \#1. Figure 41 shows the Fourier analysis without sextupoles. The $\mathrm{Q}_{\mathrm{z}}$ and $\mathrm{Q}_{\mathbf{x}}$ resonances are clearly visible. The residual coupling is very small in both planes (amplitude ratio $10^{-4}$ ). The coupling is caused by the Siberian Snake.

Figure 42 shows the simulation with sextupoles. Harmonics and mixing resonances are clearly visible. The coupling between the $\mathrm{x}$ and $\mathrm{z}$ plane is strong due to the working point of $\mathrm{Q}_{z}=0.75$. This is an octupole resonance. This working point can cause perturbations of the particle trajectory.

Case \#2. In Fig. 43 the Fourier analysis is performed for an integrated Siberian Snake with sextupoles on. The working point is away from the optical resonances. The $Q_{x}$ and $Q_{z}$ resonances are clearly visible together with harmonics and mixing frequencies. The later are very weak.

Siberian Snake, Possibility \#2. In this case the effect of the existing Siberian Snake [15] was simulated. In Figs. 44 and 45, the Fourier analysis is performed with and without sextupoles. The figures show the small residual coupling (Fig. 44) and the harmonics and mixing frequencies caused by sextupoles (Fig. 45). Both harmonics and mixing frequencies are small.

\section{Simulations of the Spin Motion with Disturbed Optics}

In the following, simulations are described in which the optics are disturbed at an energy of $a \gamma=\mathrm{n}$. The simulations were performed with errors in the magnet position and field errors (position and rotation errors in between 0.001 percent and 1 percent). 
The errors lead to additional spin rotations around arbitrary axes in space. The direction of the spin vector changes from revolution to revolution. The perturbation can coinpensate the collective spin rotation of a partial Snake. The spin directions are mixed.

\section{Simulations of the Amplified Stern-Gerlach} Effect for IUCF

The simulations were performed with a $Q_{z}$ value of $0.7(a \gamma=2)$. Figure 46 shows the $\mathrm{Q}$-values averaged over one revolution. The $\mathrm{Q}_{z}$ value is oscillating within a relatively large range. This is caused by the uncompensated chromaticity in IUCF. Nevertheless, at least for part of the time, the $Q$-value fulfills the resonance condition and a separation should be possible. Figure 47 confirms this. The figure describes the position in $z$ and the angle between the spin vector and the $z$-axis.

\section{Summary}

Simulations of the repetitive Stern-Gerlach effect were performed for various machine conditions. It was shown that a small $\mathrm{Q}$-spread was not required. An oscillation around the resonance is necessary for separation. The smaller the Qspread of the particles, the stronger the separation. 


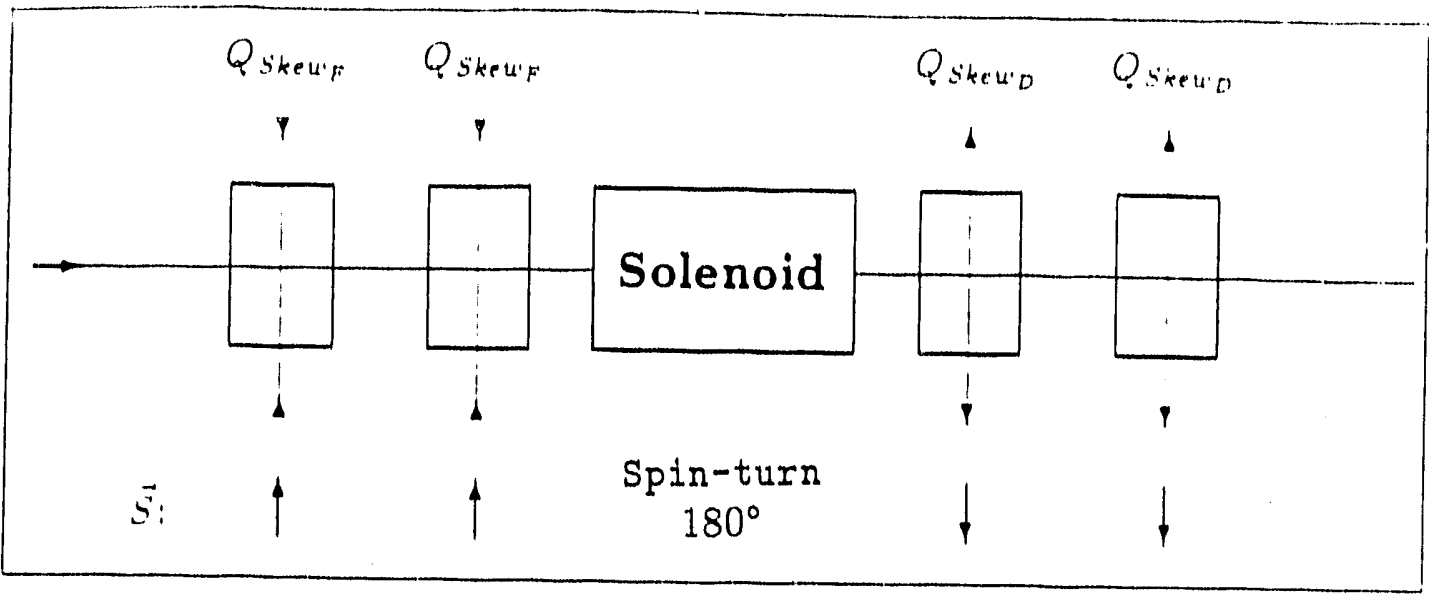

Figure 21. Schematic of the Spin-Splitter consisting of two skew quads $Q_{F}, Q_{D}$ and a solenoid, the length of the quadrupoles is between $0.25 \mathrm{~m}$ and $0.5 \mathrm{~m}$, the length of the soelnoid is between 0.32 and $1.6 \mathrm{~m}$, the distance is between $0.1 \mathrm{~m}$ and $1.0 \mathrm{~m}$, and the total maximum length of the unit is $6 \mathrm{~m}$.

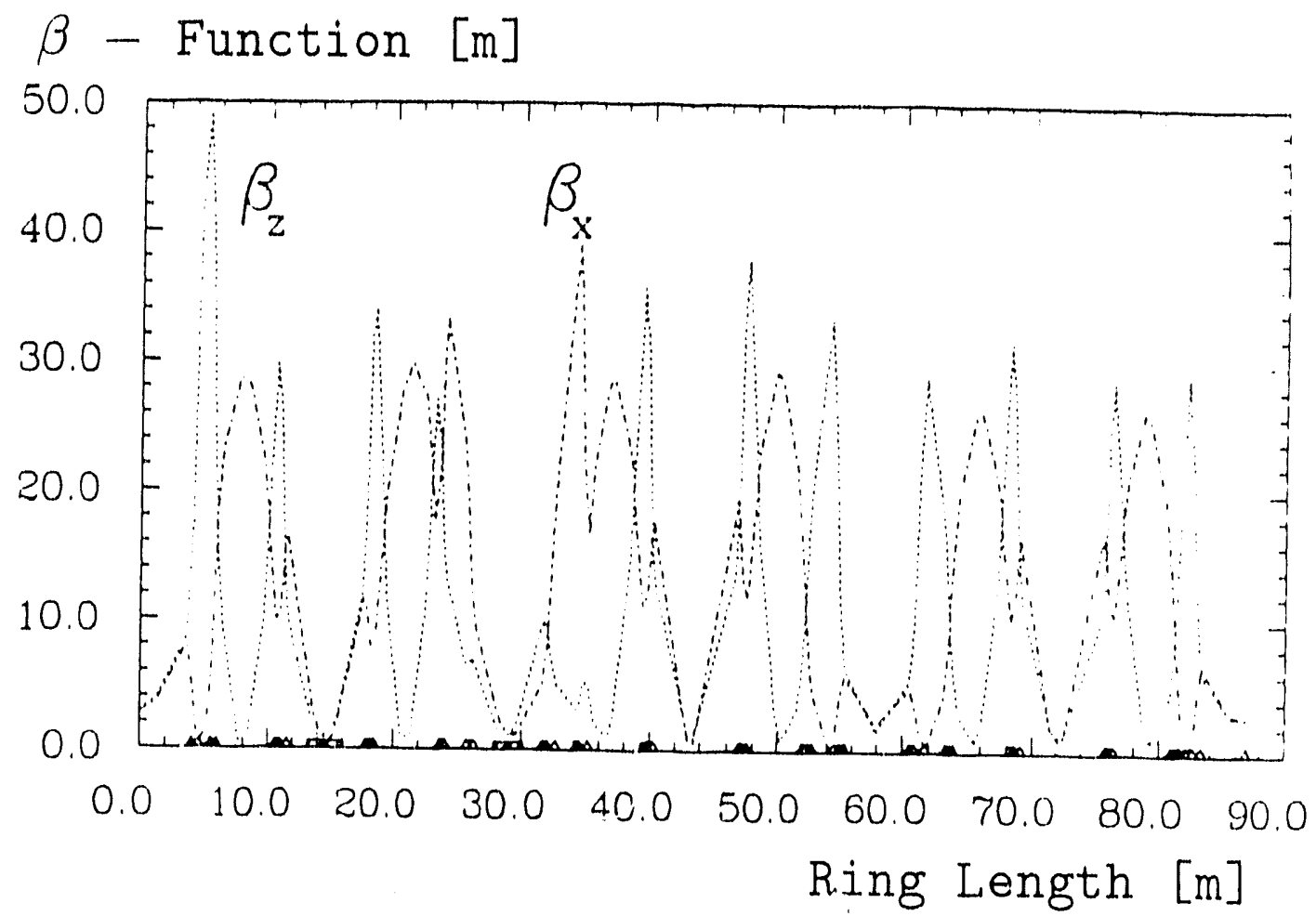

Figure 22. $\beta$-function of the unchanged IUCF ring (triangles, quads, squares, and bending magnets). 


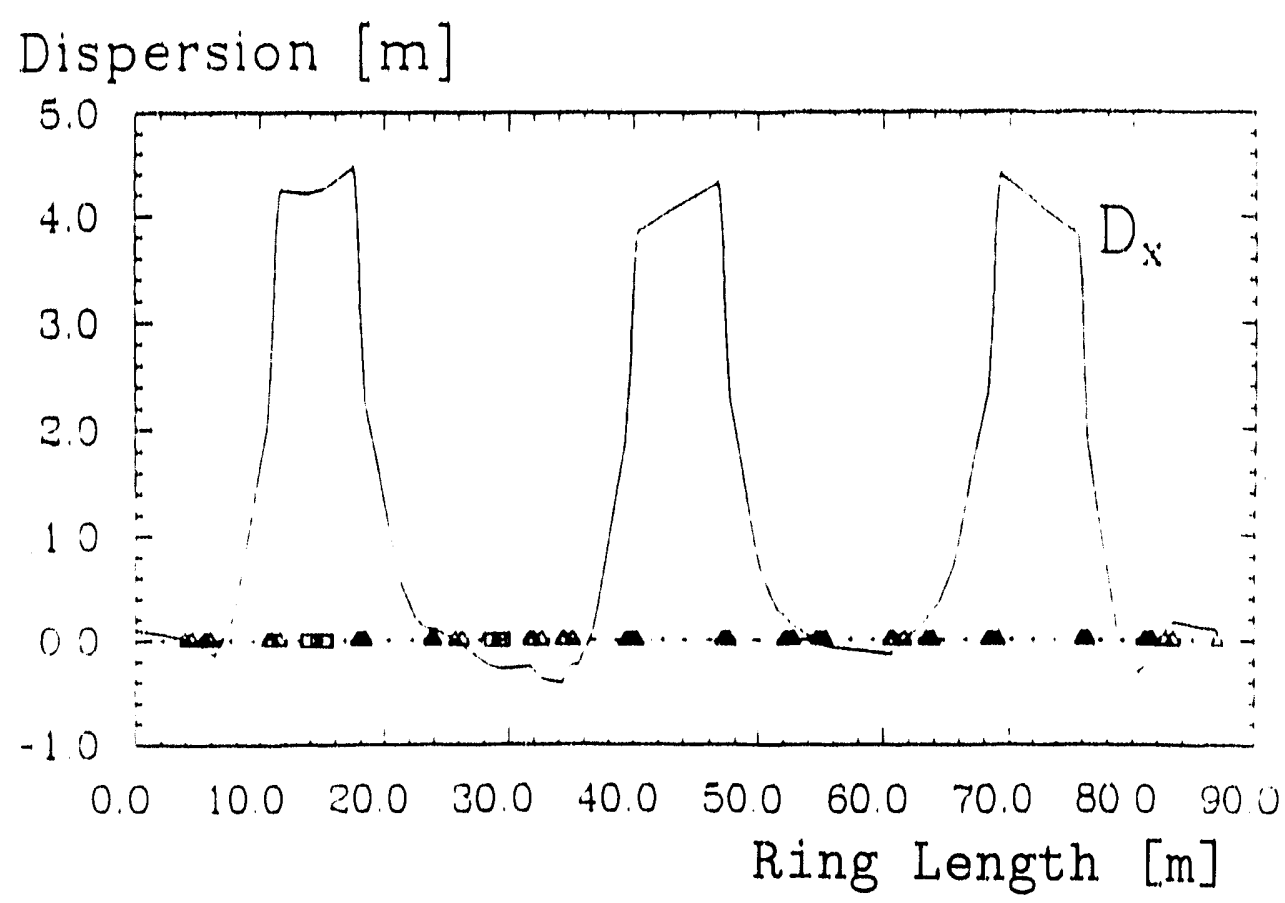

Figure 23. Dispersion of the unchanged IUCF ring (triangles, quads, squnres, and bending magnets).

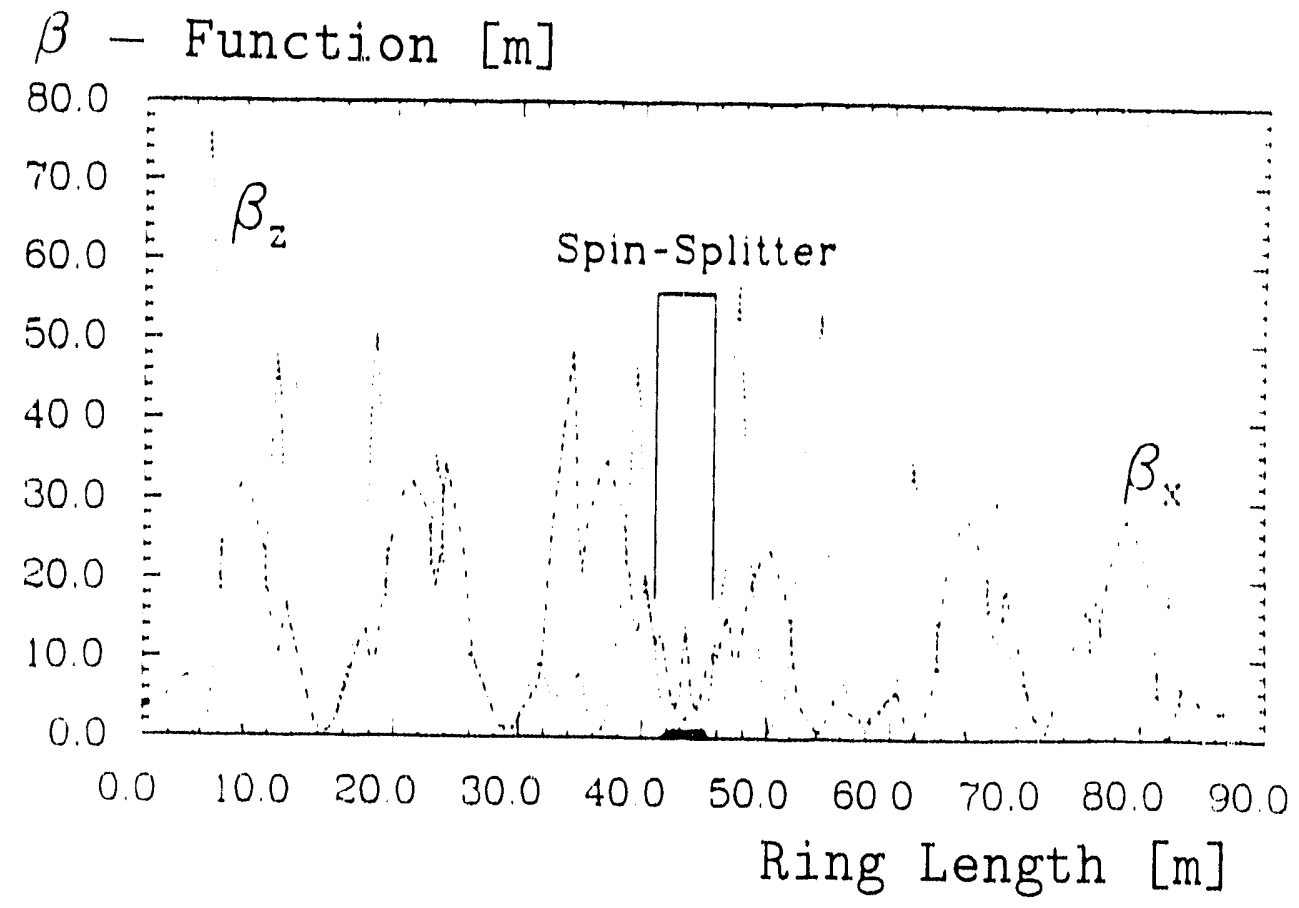

Figure 24. $\beta$-function of the IUCF ring with integrated Spin-Splitter (triangles, quads, dots, and solenoid). 


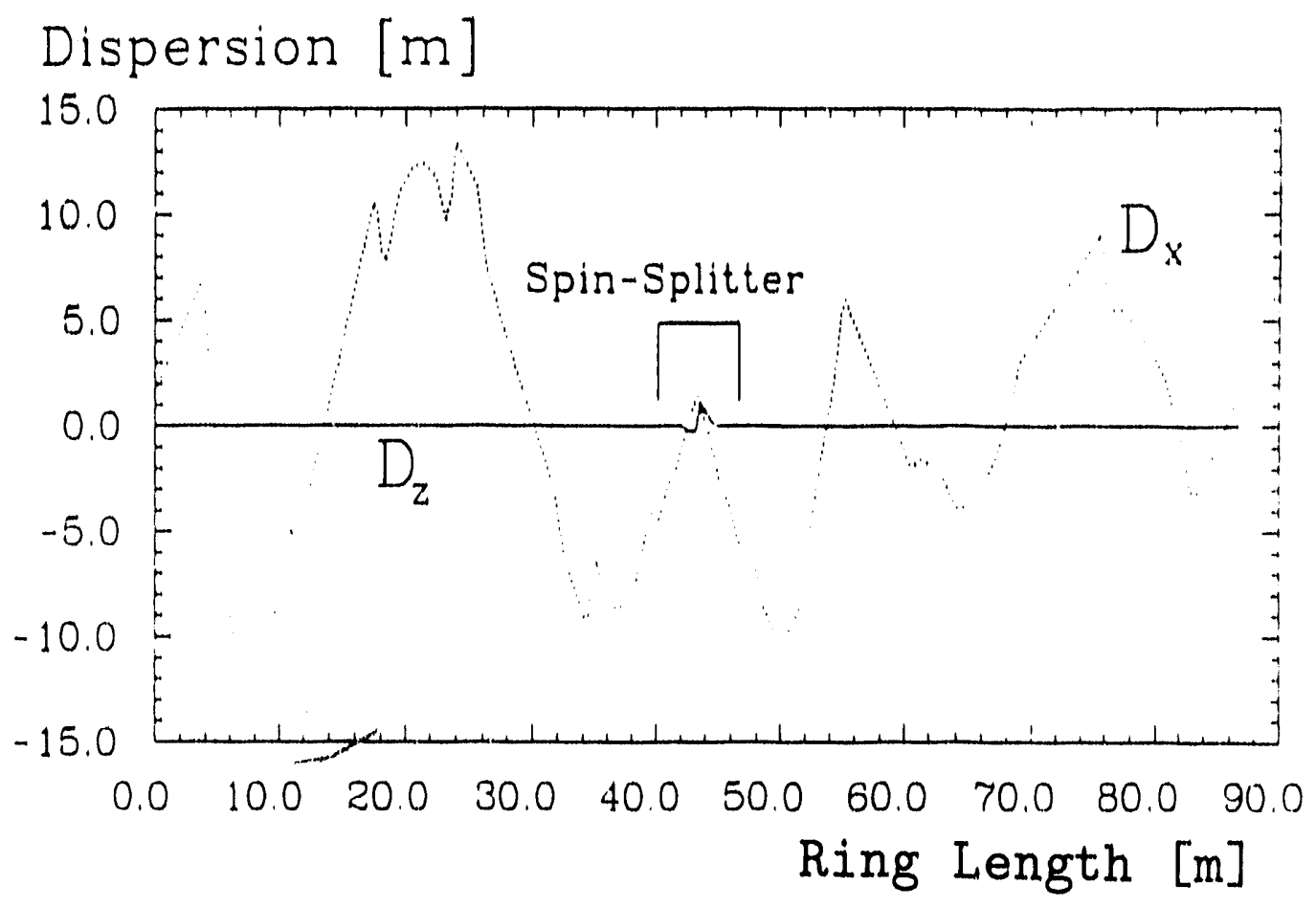

Figure 25. Dispersion of the IUCF ring with the integrated Spin-Splitter.
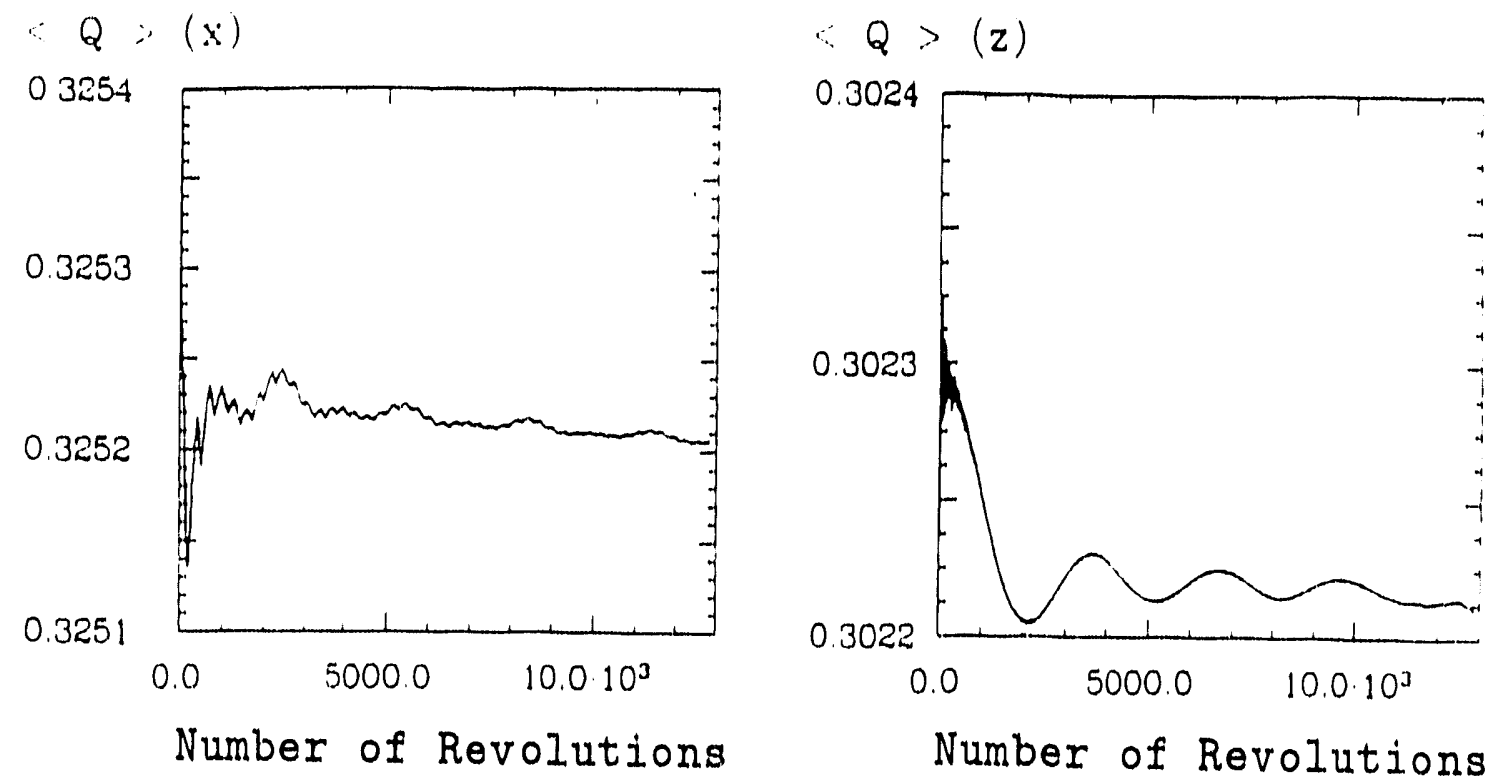

Figure 26. Averaging the Q-values over many revolutions ( $Q$-values for $x$ and $z$ ). After several initial oscillations, the final value is reached. 

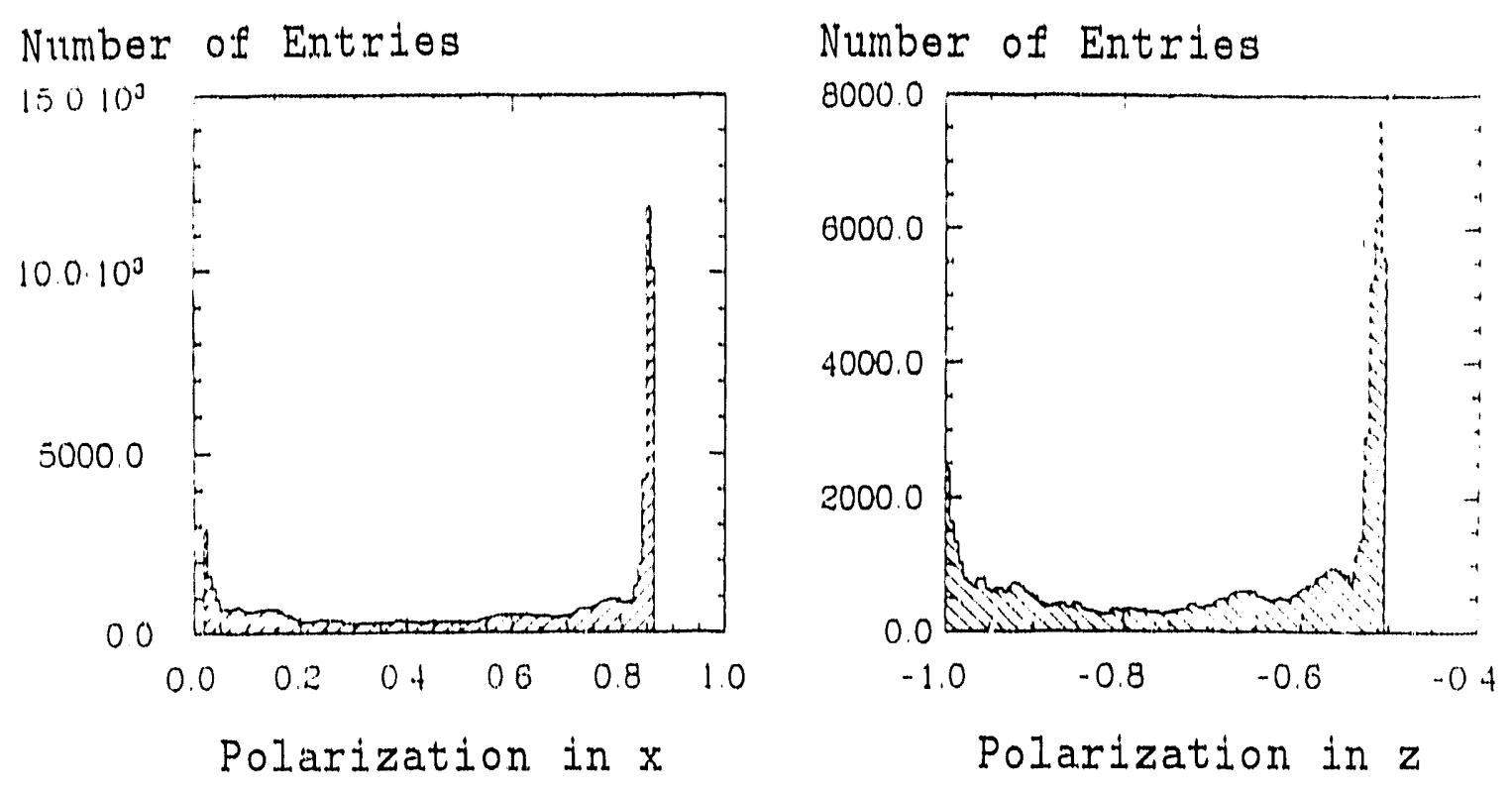

Figure 27. Distribution of the $x$ und $z$ components of the spin after 60,000 revolutions.
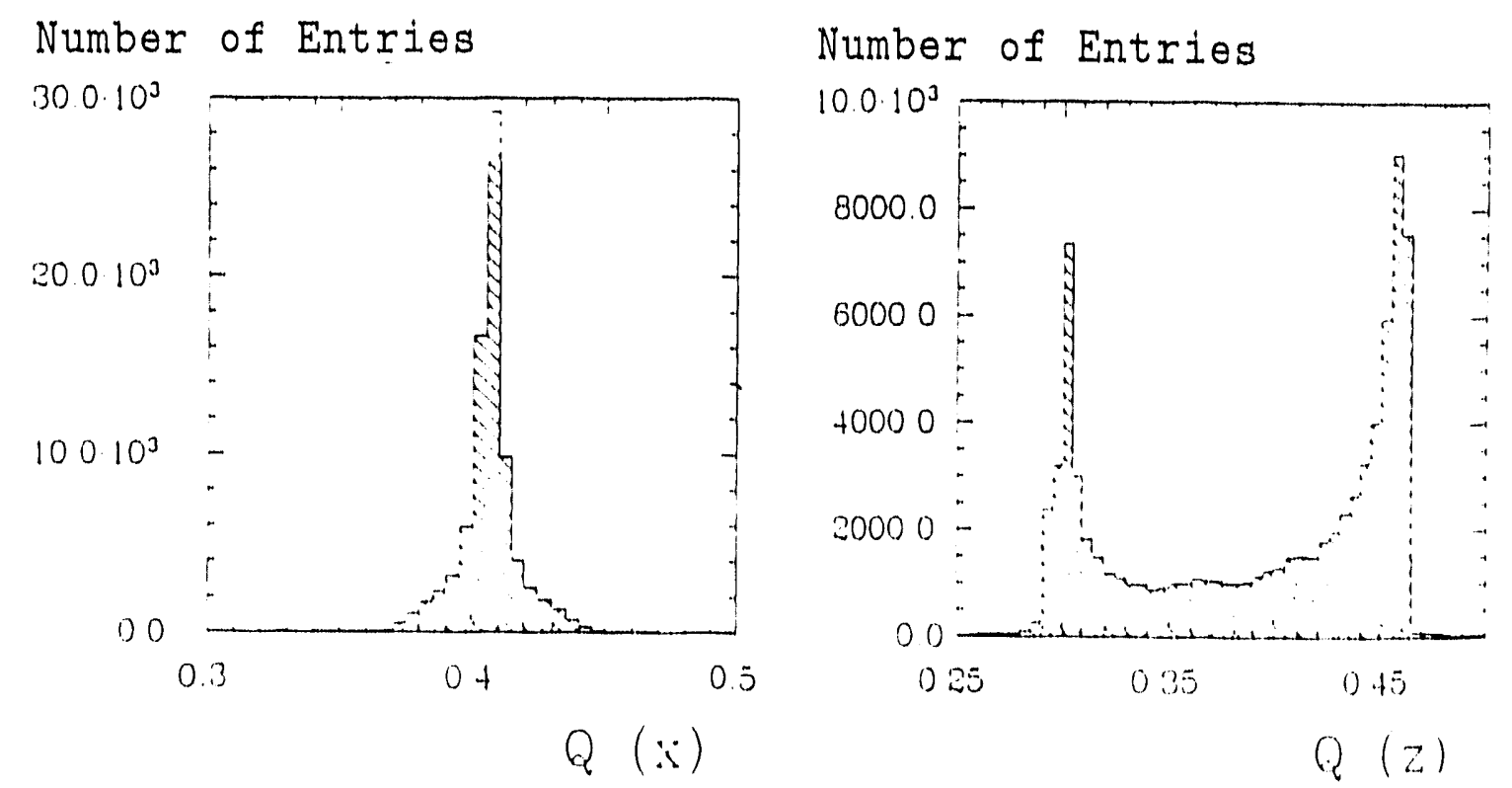

Figure 28. Distribution of the Q-values for $x$ and $\%$ A cleur brondening of the distribution for 102 particles after 60,000 revolutions can be secn. 


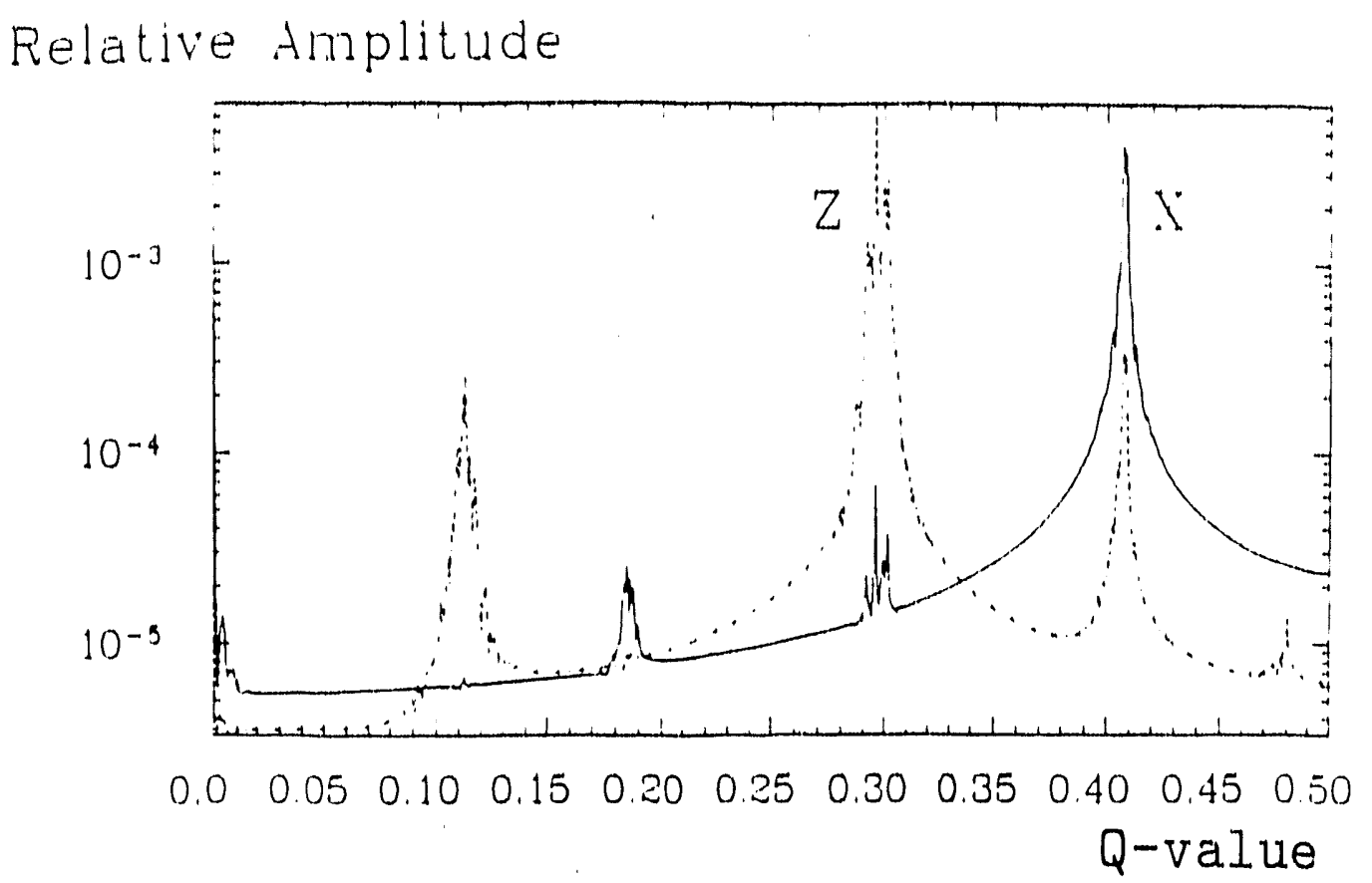

Figure 29. Fourier analysis of the particle motion for the disturbed optics (error in magnet positioning, magnet rotation and field errors). In addition to the main frequencies, the errors produce side bands.
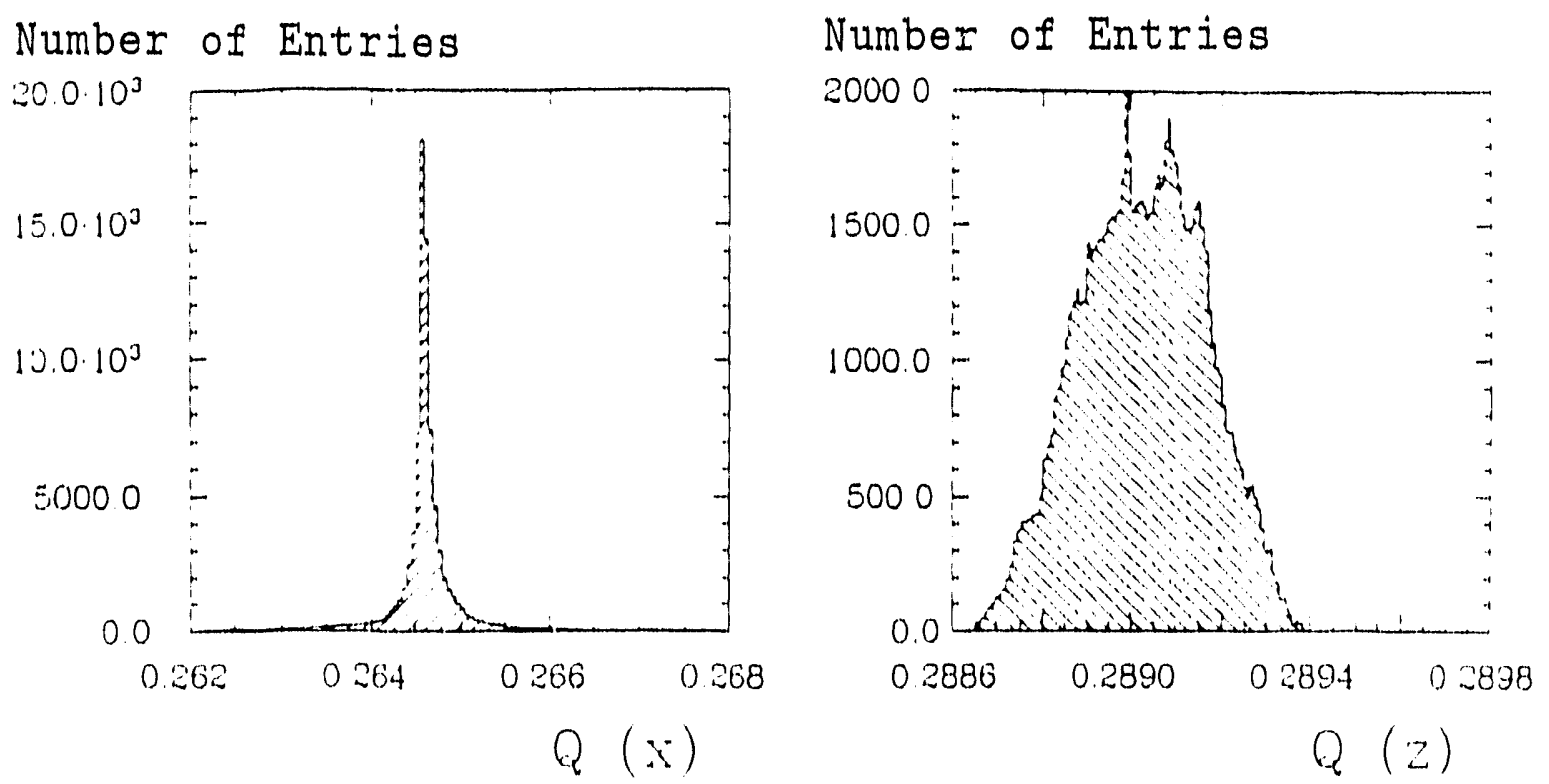

Figure 30. Distribution of the Q-values for 800 revolutions. The average values coincide with the the linear predictions. 

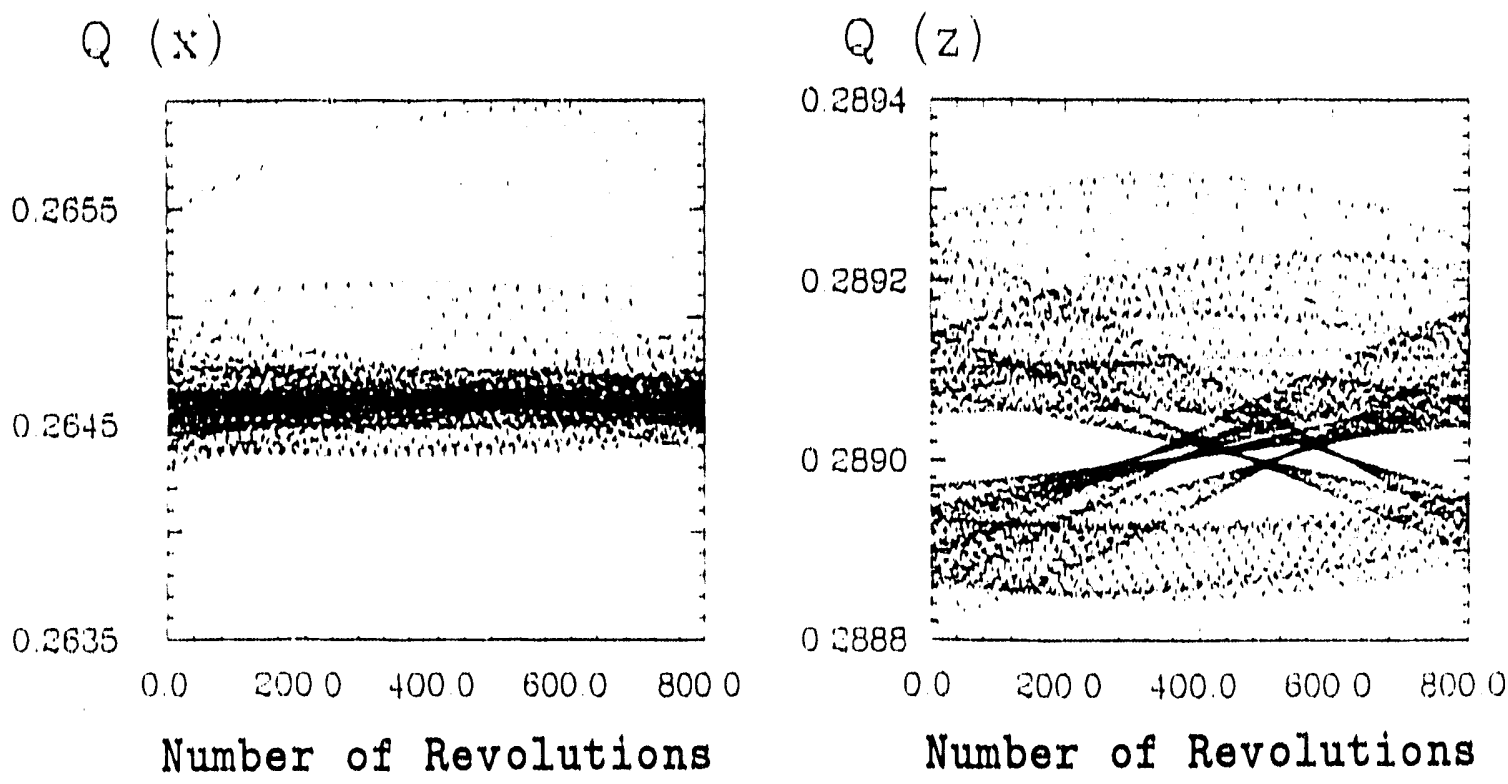

Figure 31. Oscillation of the Q-values for 10 different particles. The Q-value of the particles oscillates arou d an average value.

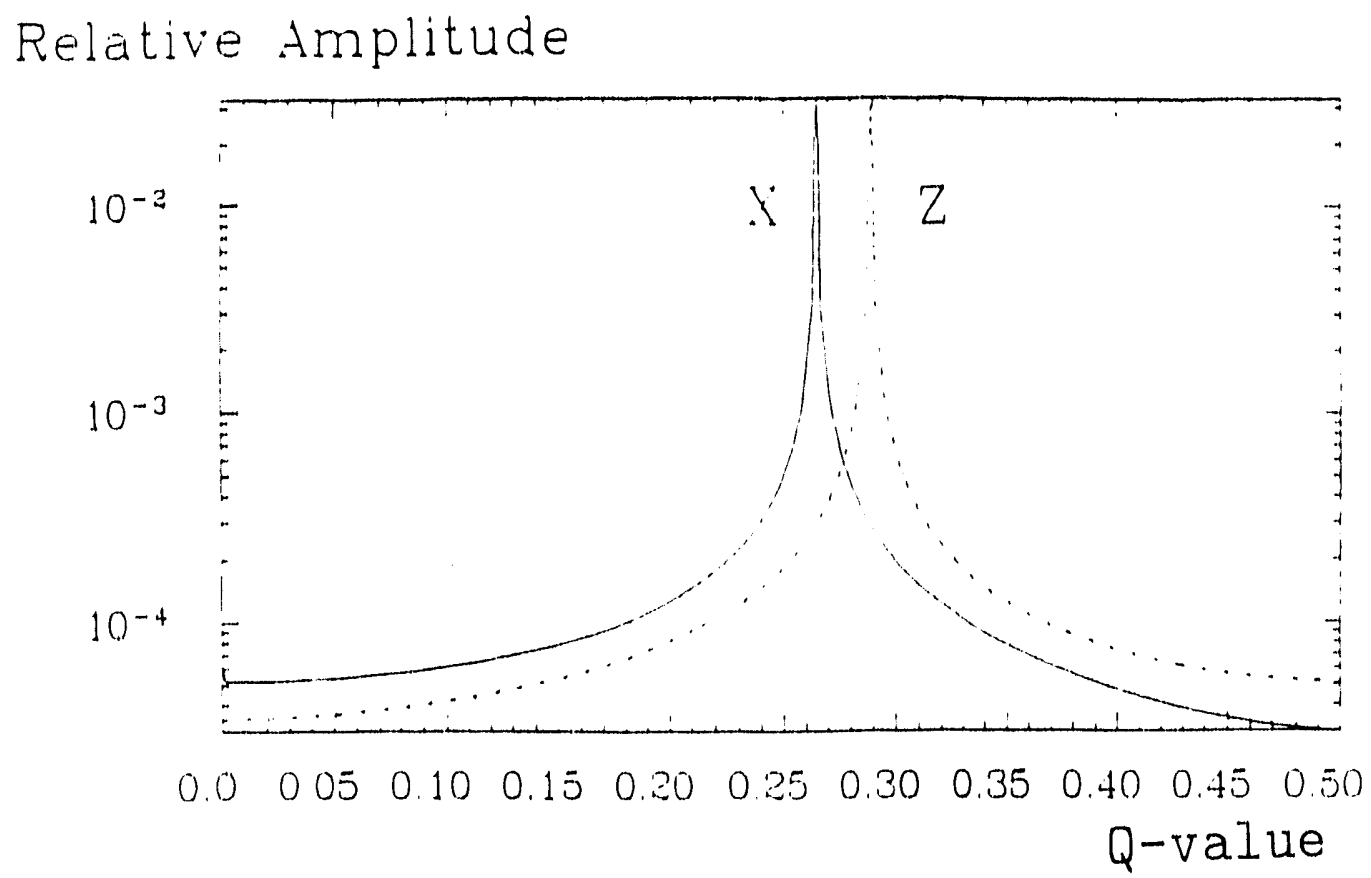

Figure 32. Fourier analysis of the particle oscillations. 


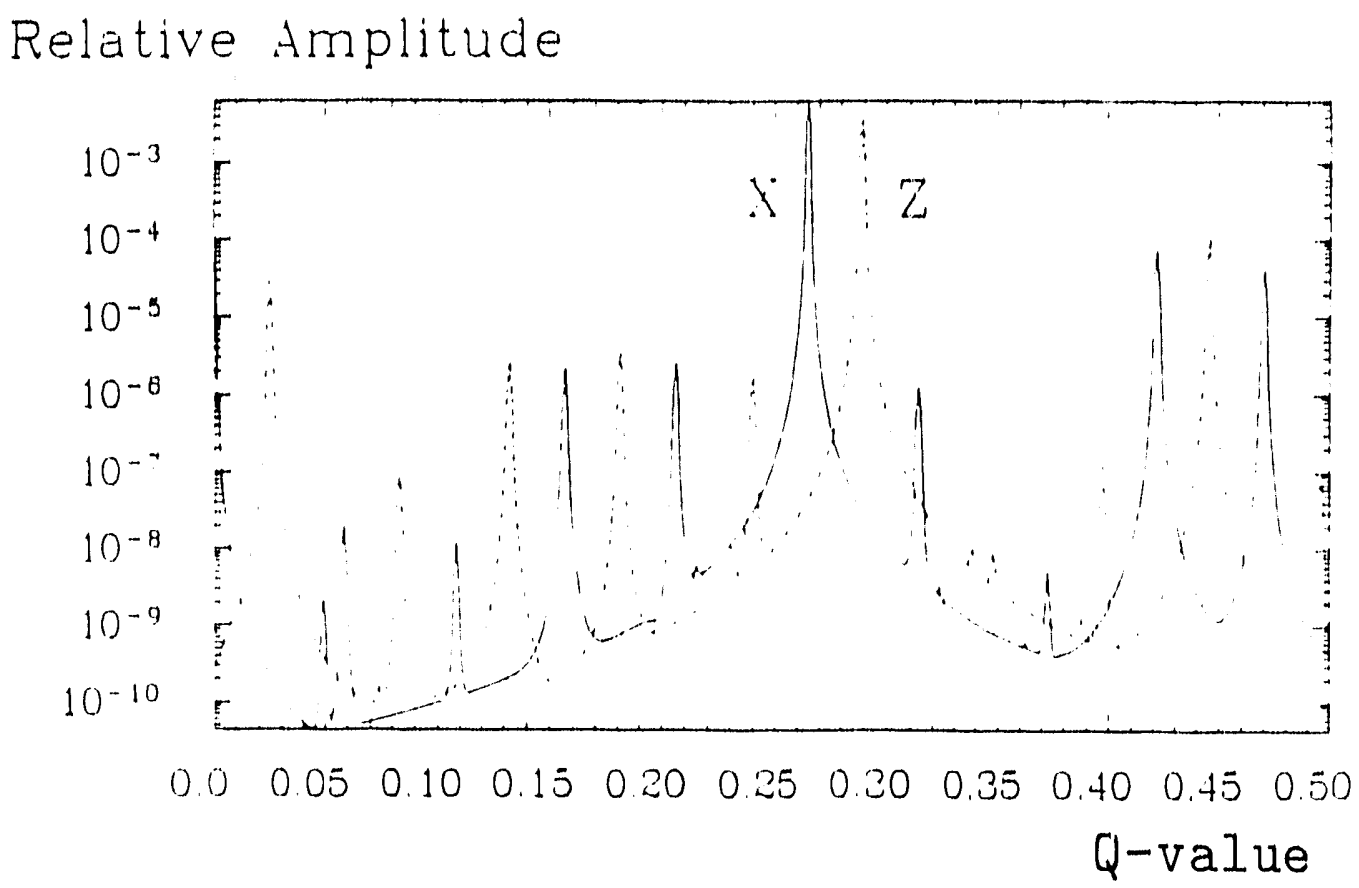

Figure 33. Fourier spectrum of the particle trajectories with sextupoles on. The additional harmonics and the frequency mixing are clearly visible.
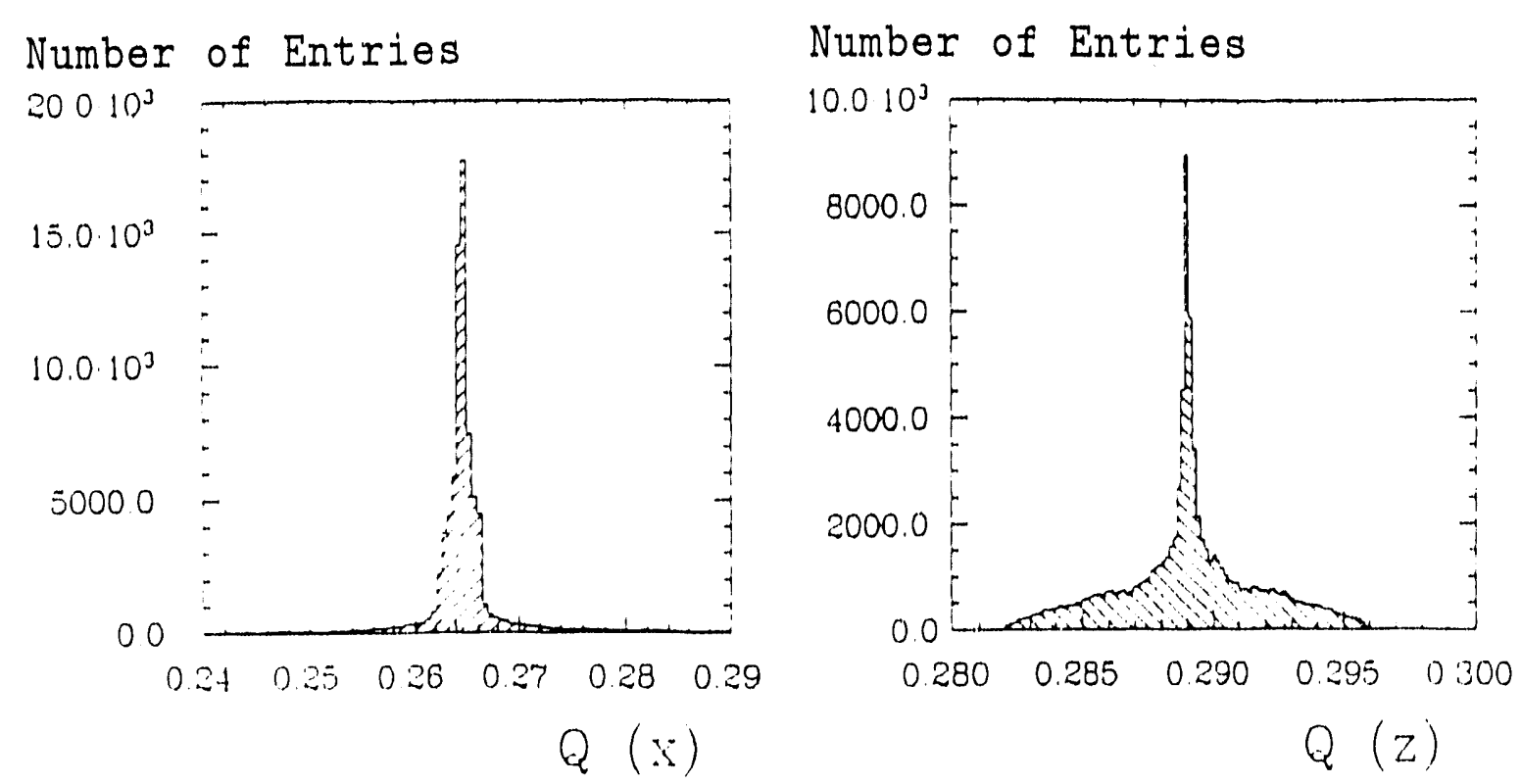

Figure 34. Distribution of Q-values over 600 revolutions after 70,000 revolutions. 

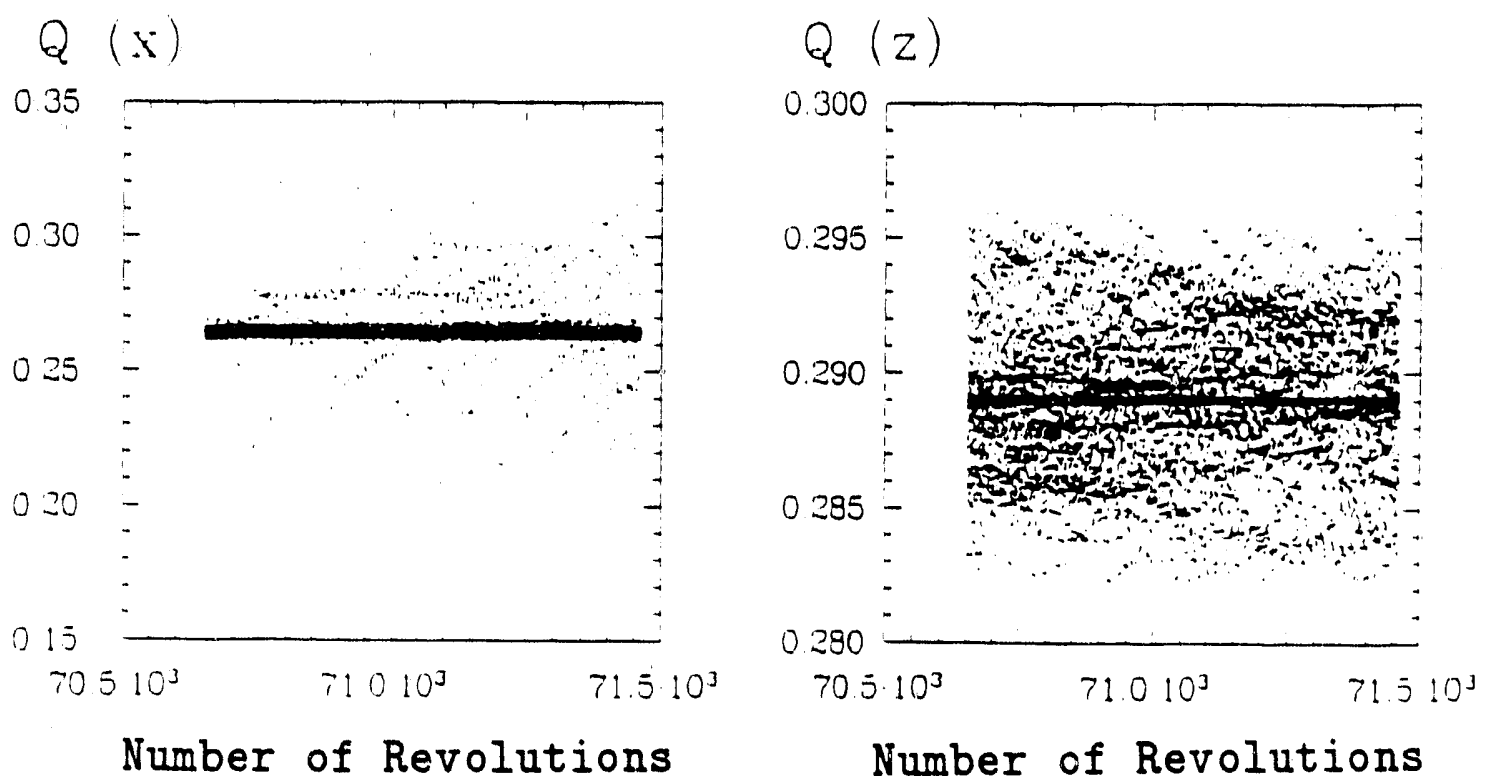

Figure 35. Oscillation of Q-values for 10 particles over 800 revolutions. It is obvious that the particles oscillate around an average $\mathrm{Q}$-value.

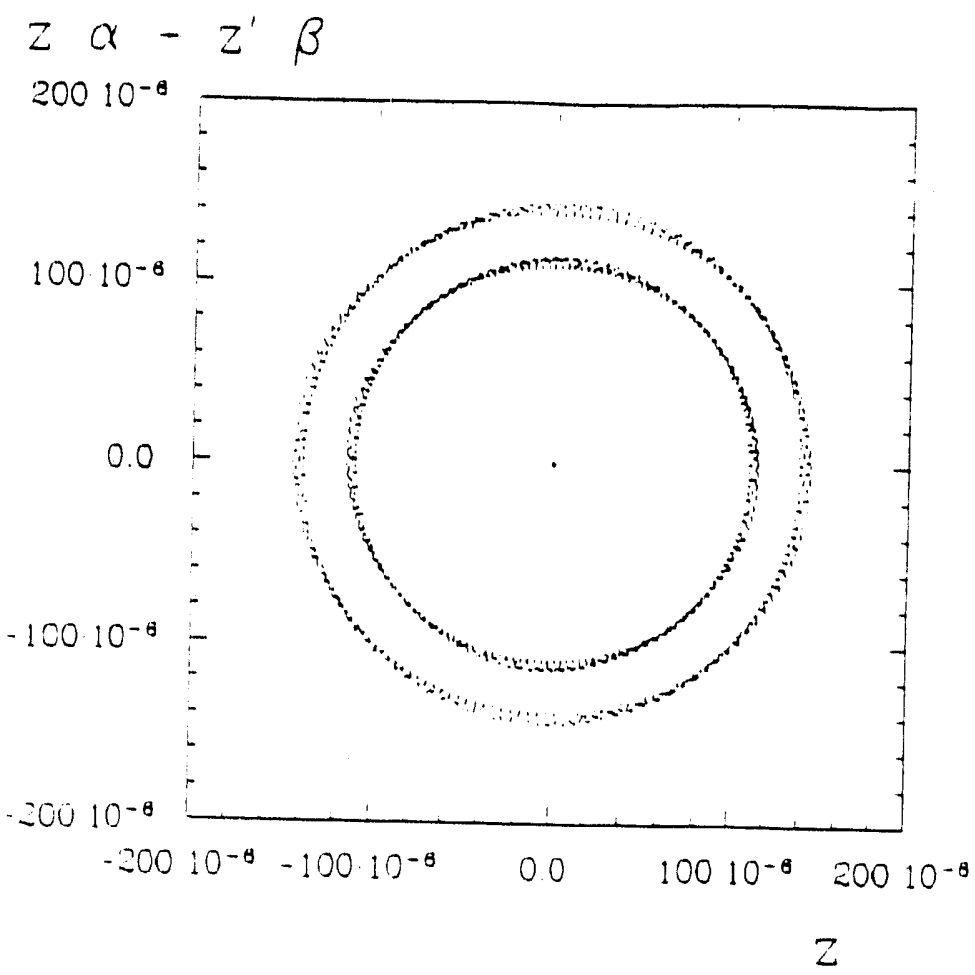

Figure 36. Vertical phase ellipse of the IUCF ring with sextupoles. 


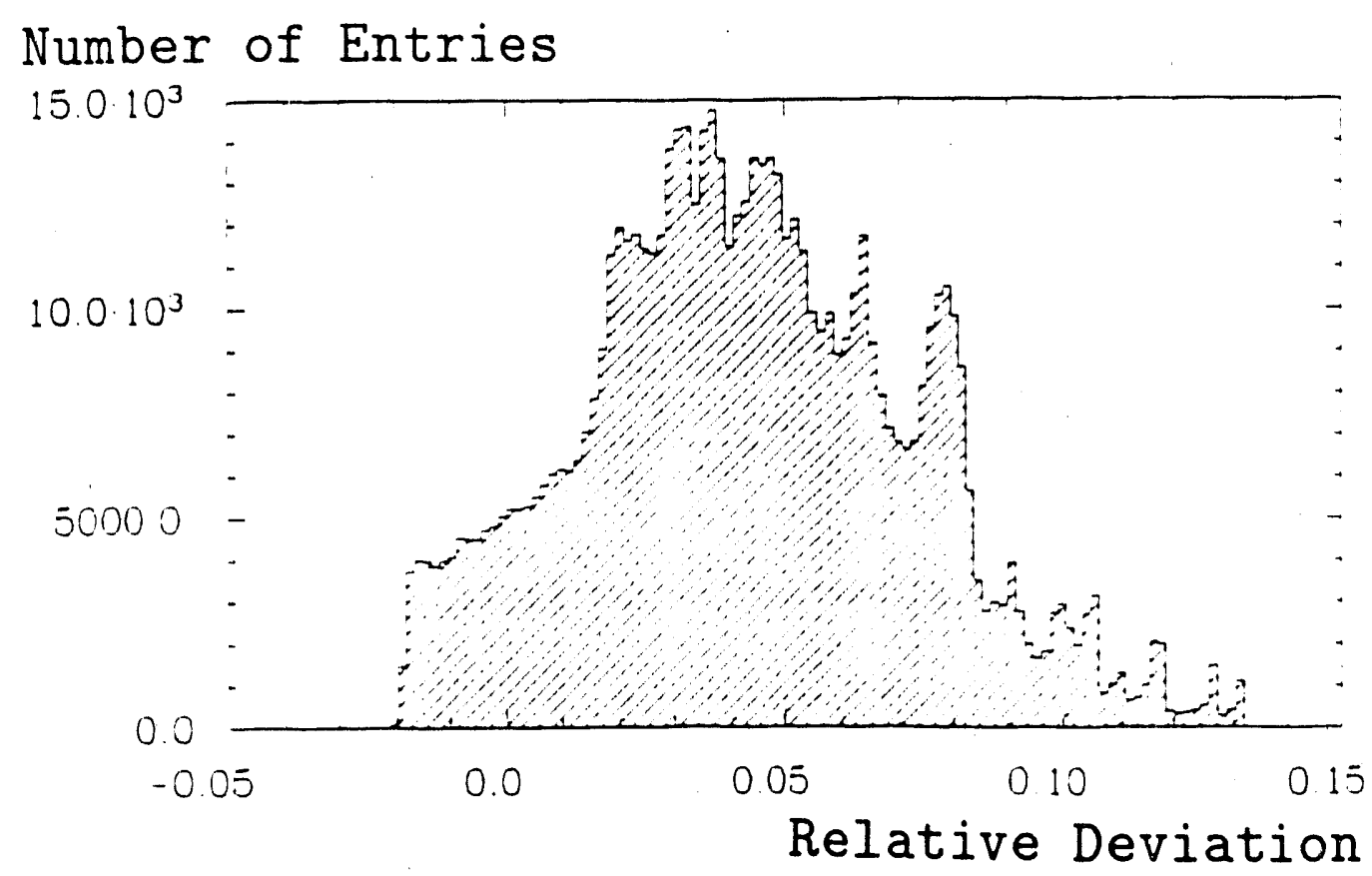

Figure 37. Difference between the length of the closed orbit and the length of the actual particle trajectory. The distribution has a maximum close to 0.04 .
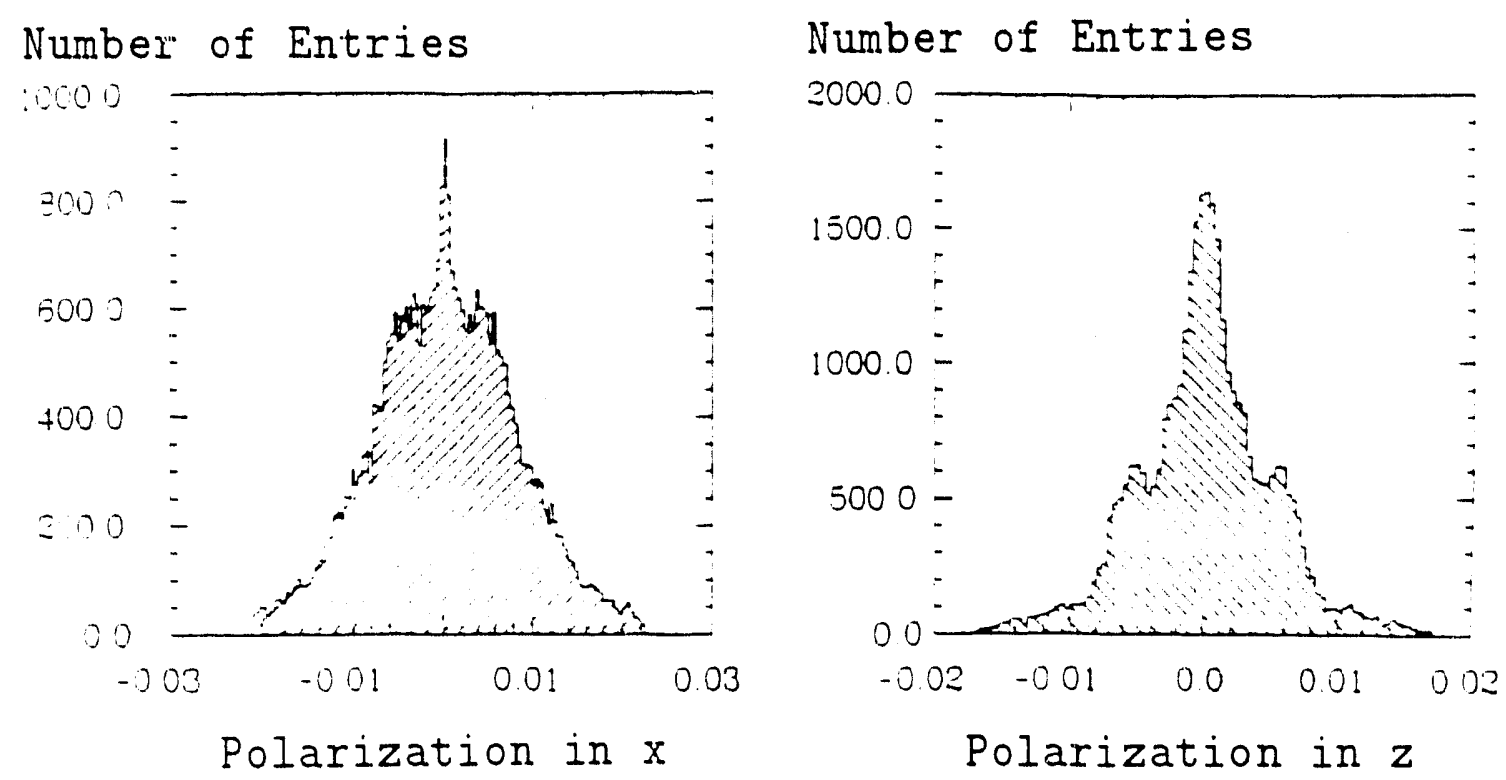

Figure 38. Distribution of the spins in the $\mathrm{x}$ and $\mathrm{z}$-direction after 50,000 revolutions. The original spin direction is parallel to the $n$-axis. The distribution shows small $\mathrm{x}$ and $\mathrm{z}$ components caused by the $\mathrm{n}$-axis outside the horizontal plane. There are no signs of depolarization. 


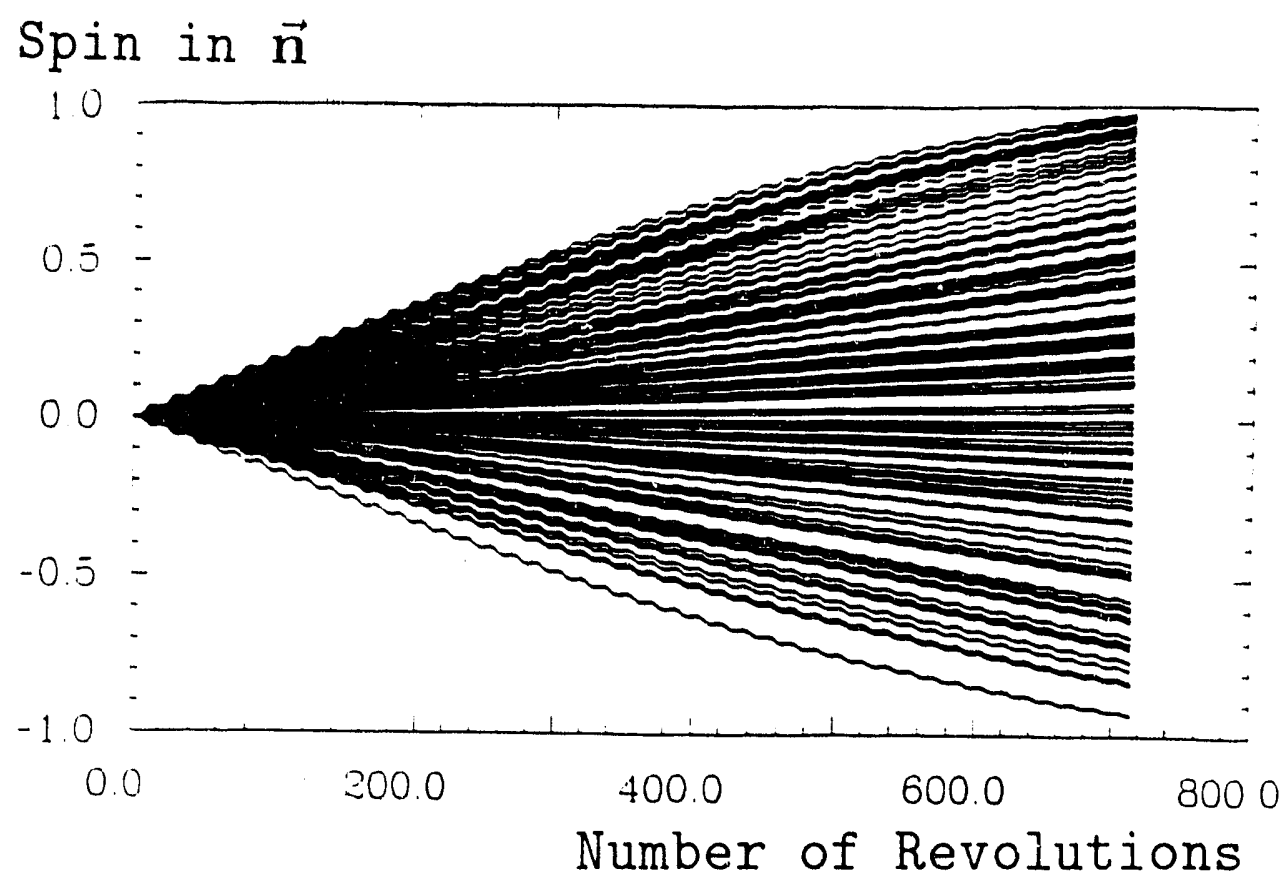

Figure 39. Component of the polarization parallel to the $\mathrm{n}$-axis for a $\mathrm{Q}_{\mathrm{z}}=\nu_{\mathrm{s}}=0.47$ resonance. The beam is depolarized within a few revolutions. $\mathrm{P}(\mathrm{z})=-1$.

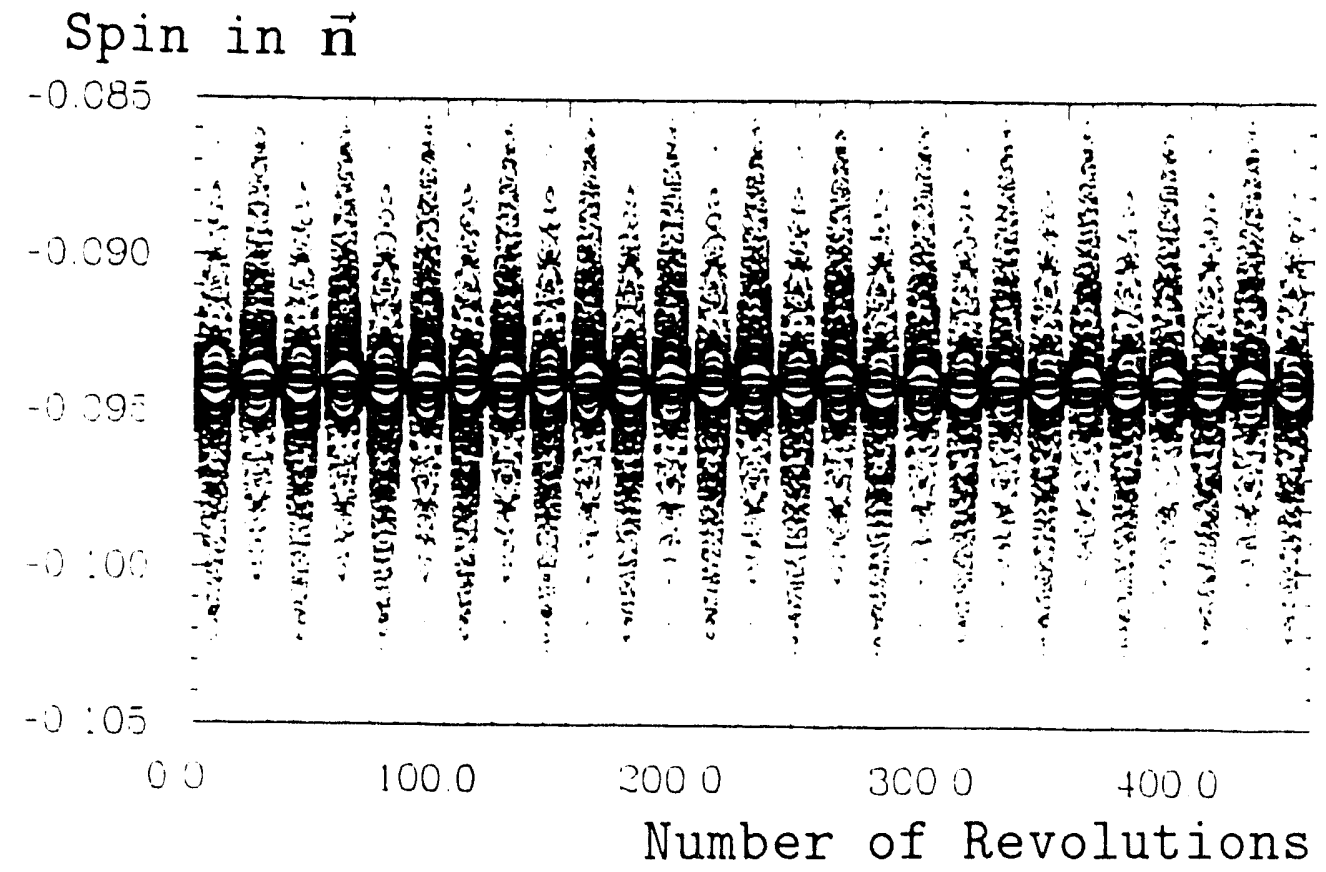

Figure 40. Component of the polarization parallel to the $\mathrm{n}$-axis. $\mathrm{Q}_{\mathrm{z}}=\nu_{\mathrm{s}}=0.47$, $\mathrm{a} \gamma=2.5$. There is no depolarization. $\mathrm{P}(\mathrm{z})=-1$ at the beginning. The component parallel to $n$ remains constant. 


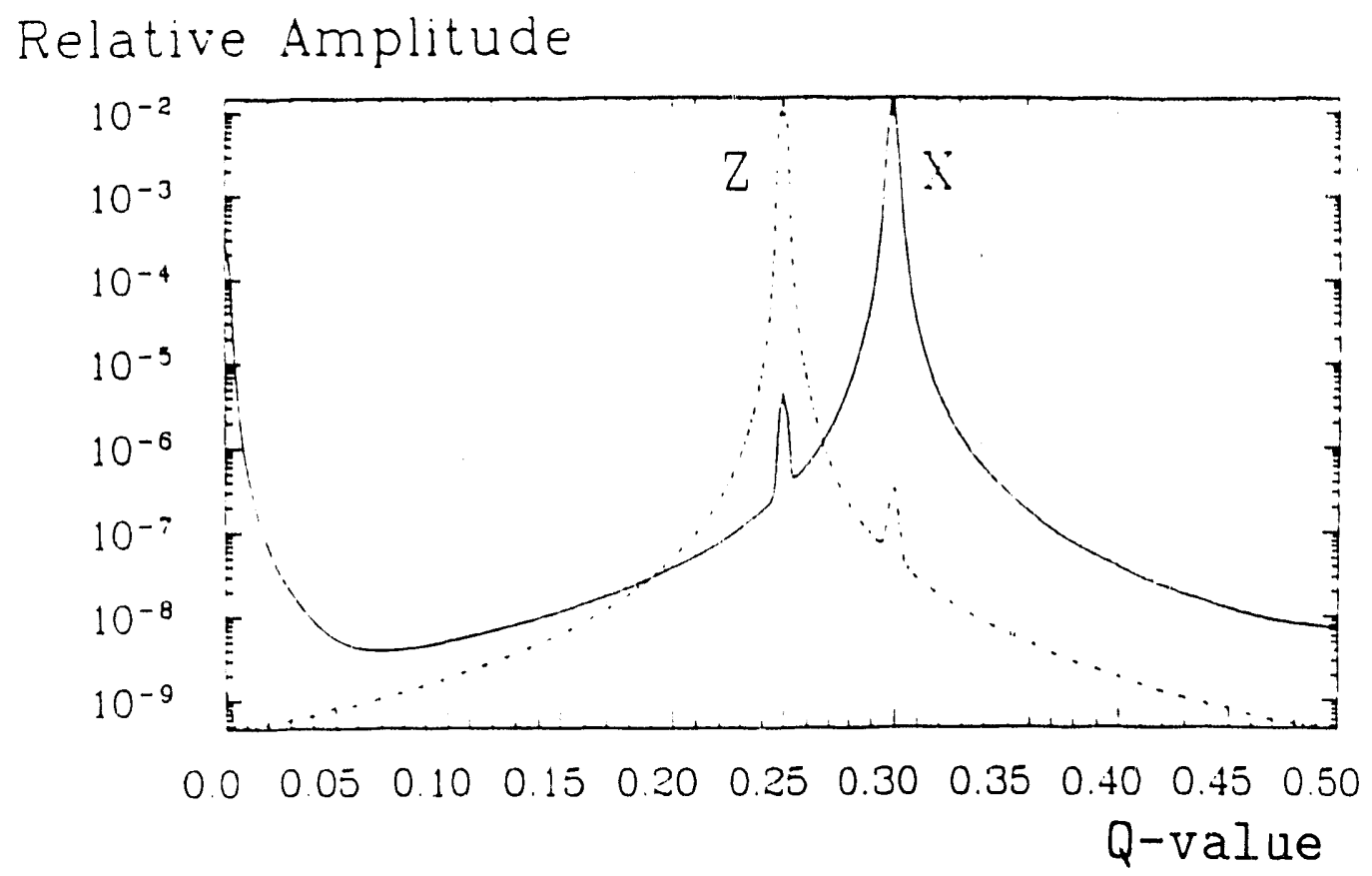

Figure 41. Fourier analysis of the particle trajectories with the Siberian Snake and without sextupoles. The $\mathrm{Q}_{\mathrm{x}}$ and $\mathrm{Q}_{\mathrm{z}}$ resonances are clearly visible. The residual coupling caused by the solenoid is negligible.

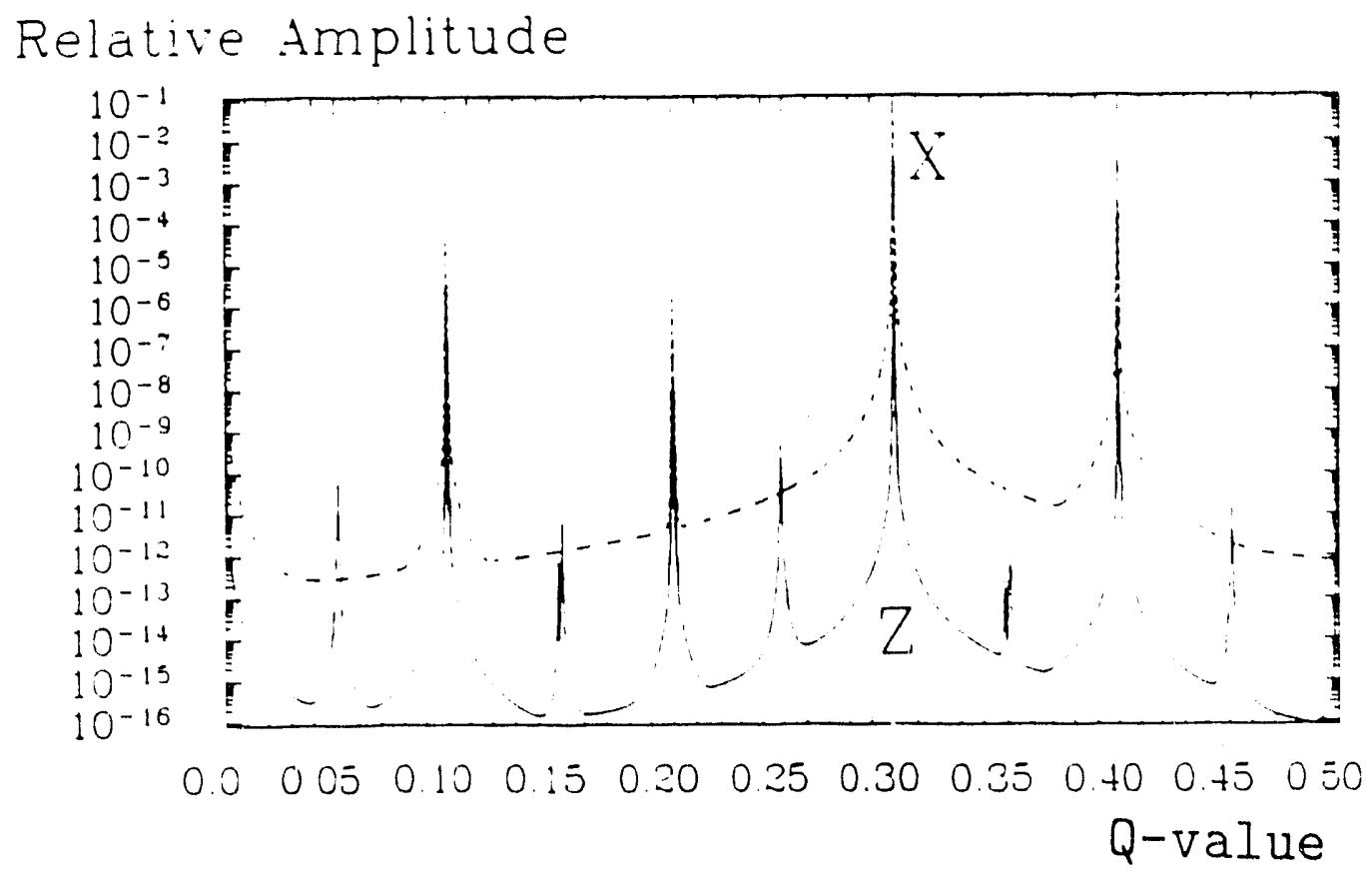

Figure 42. Fourier analysis of the particle trajectories with the Siberian Snake and with sextupoles. The working point is $Q_{z}=0.75$ (octupole resonance). The coupling is clearly visible. 


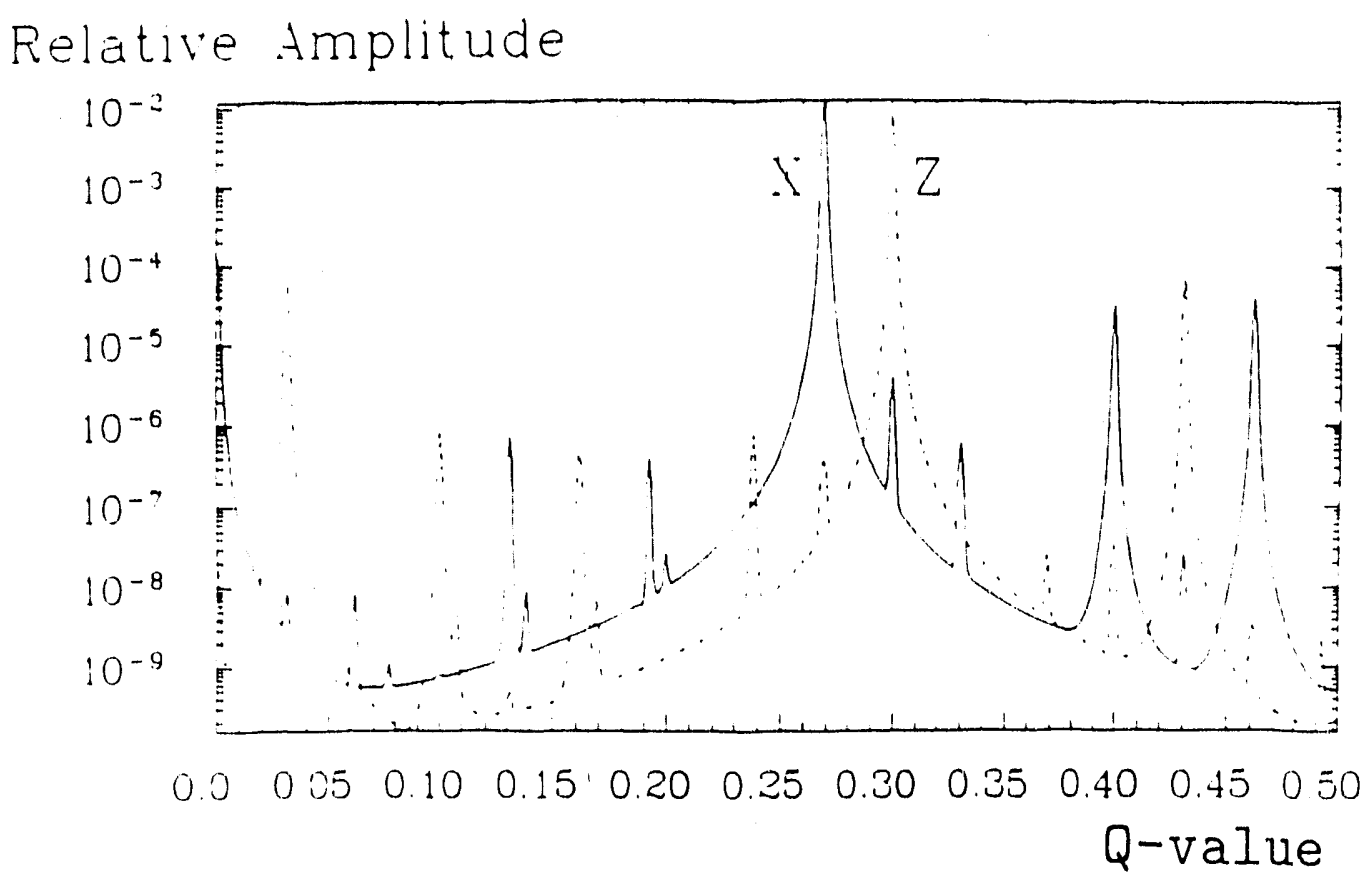

Figure 43. Fourier analysis of the particle trajectories with the Siberian Snake and sextupoles. The working point $\mathrm{Q}_{2}$ of the ring is not on the resonance and there is no strong coupling.

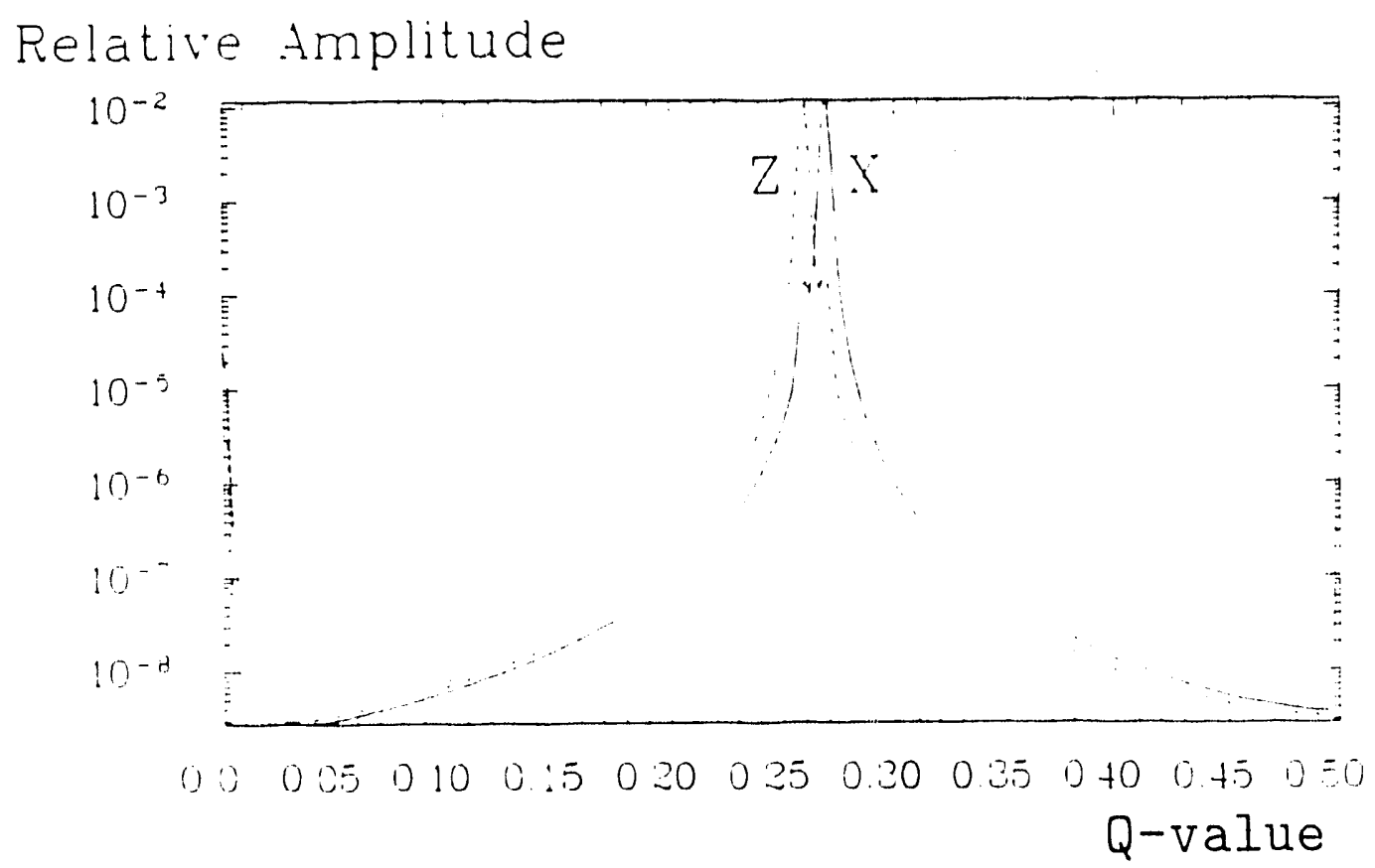

Figure 44. Fourier analysis of the particle trajectories with the Siberian Snake but without sextupoles. The residual coupling is clearly visible but small. 


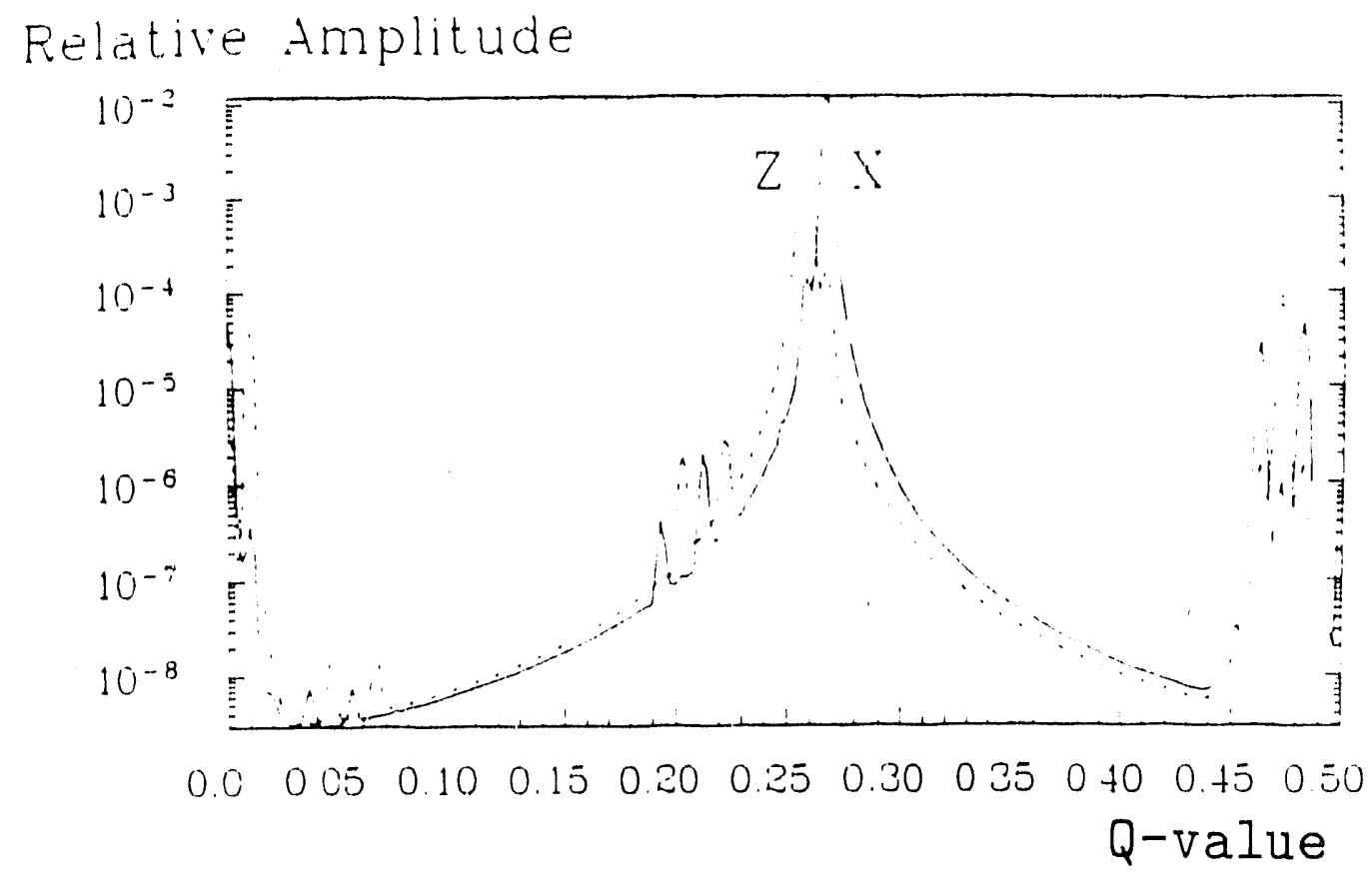

Figure 45. Fourier analysis of the particle trajectories with the Siberian Snake and sextupoles. The coupling caused by sextupoles is small and does not affect the trajectories.

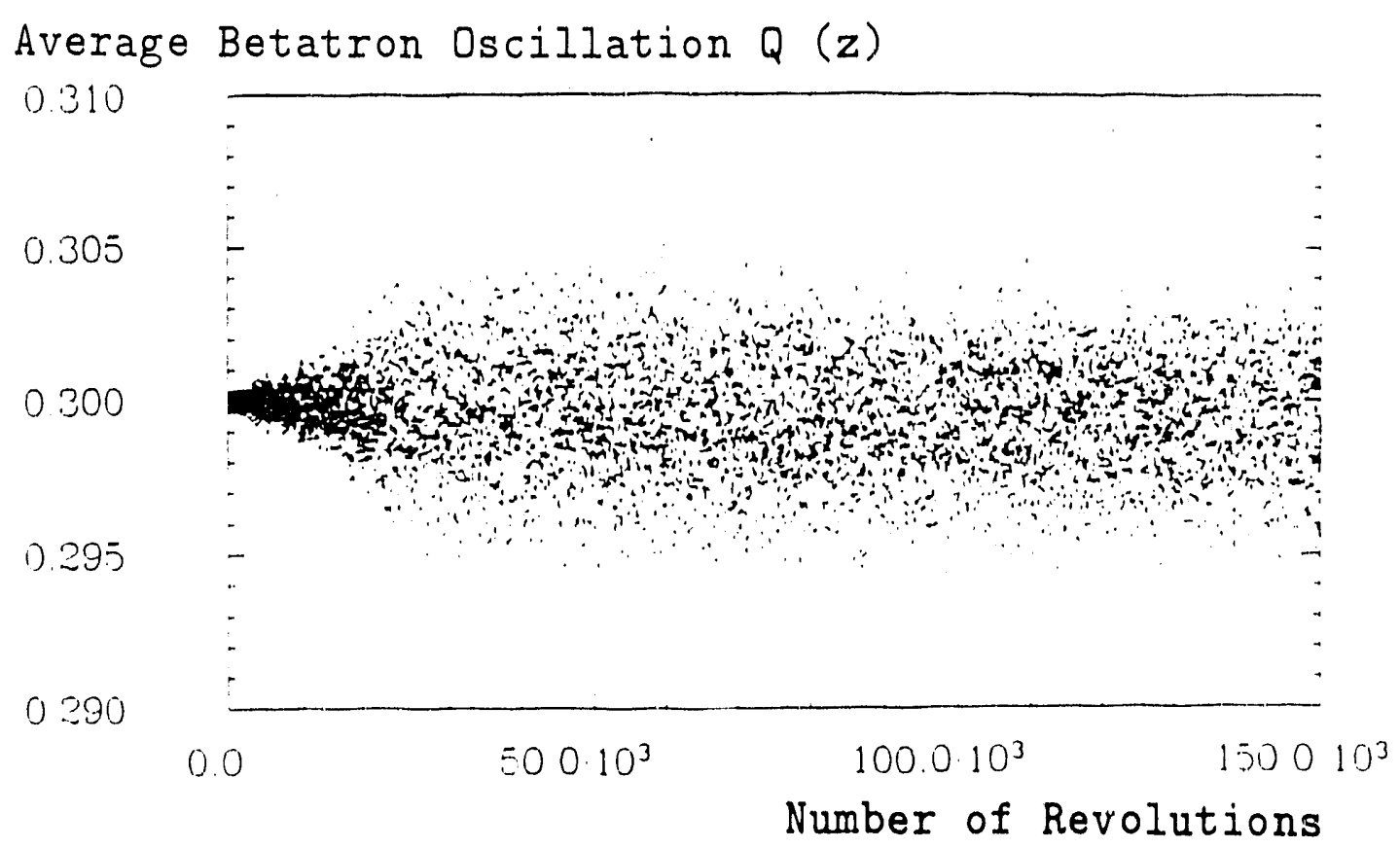

Figure 46. Averaged $Q_{2}$ value per revolution. The amplitude of the oscillations is large. 


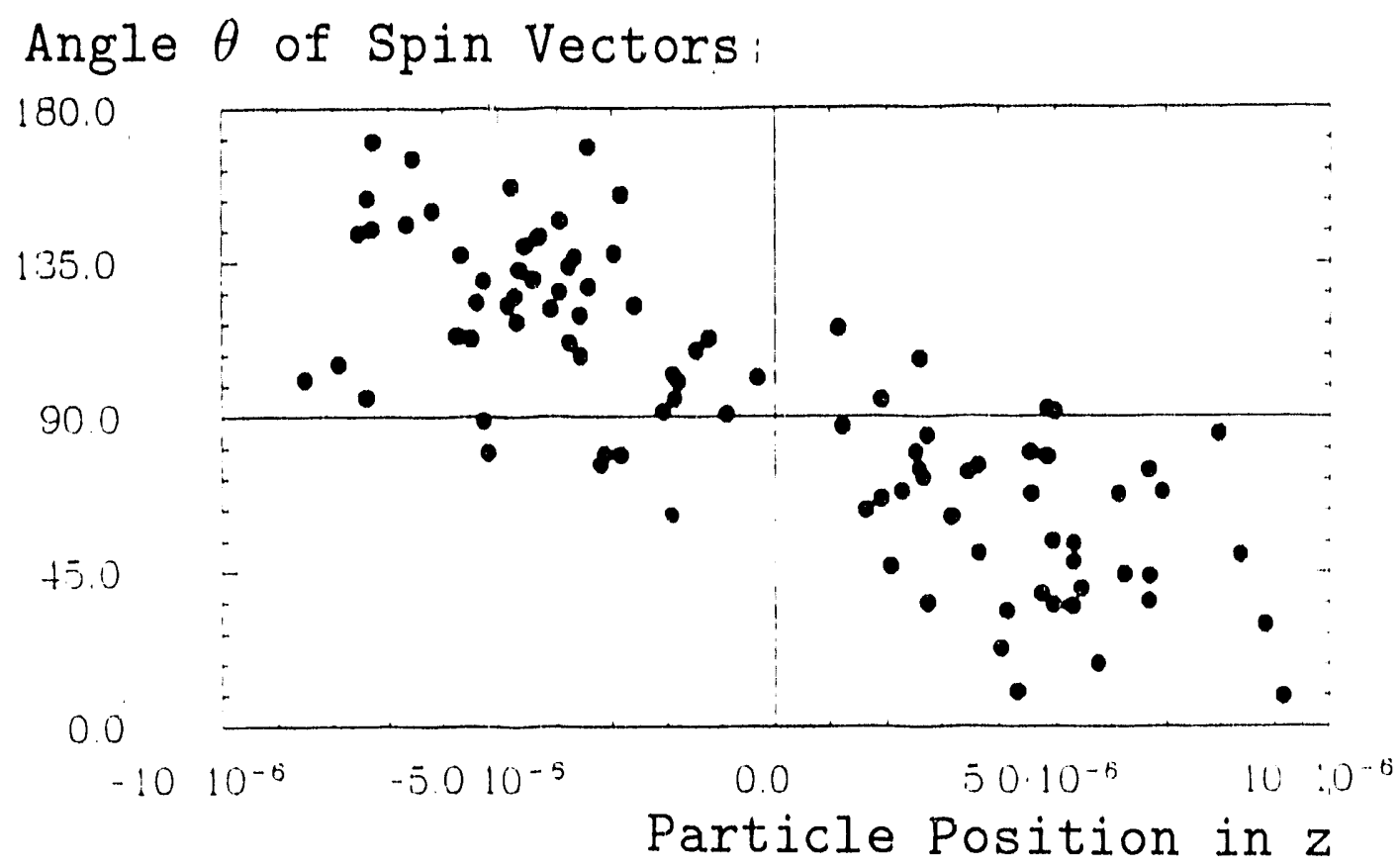

Figure 47. Position of the particles in $z$ versus the projected angle of the spin vector relative to the n-axis. The spin separation is clearly visible. 


\title{
C. Theoretical Studies
}

\author{
M. Conte, INFN and University of Genova, Italy \\ Y. Onel, University of Iowa, USA \\ A. Penzo, University of Trieste, Italy \\ A. Pisent, INFN-LNF, Padova, Italy \\ M. Pusterla, INFN and University of Padova, Italy \\ R. Rossmanith, CEBAF, USA
}

We have improved on the physics interpretation of the Spin-Splitter concept by using theoretical studies. We have performed calculations using analytical methods, and we have investigated the quantum-mechanical limitations (see the Appendix, Conte et al. and Ref. [2]). 


\section{REFERENCES}

[1] Y. Onel, A. Penzo, and R. Rossmanith, AIP Conf. Proc, 150, 1220 (1080);

T. Niinikoski and R. Rossmanith, Nucl, Instr, Meth, A 255, 460) (1087),

[2] M. Conte and M. Pusterla, Il Nuovo Cimento 103A, 1087 (1990).

[3] R. van Dyck, P. Schwinberg, and H. Dehmelt, Phys, Rev, D, 722 (1986);

Is Dehmelt, 1989 Nobel prize talk, Rev. Mod. Phys, 62, 525 (1990).

[4] A. D. Krisch et al, Phys, Rev, Lett, 63, 1137 (1089).

[5] T. Ellison, private communication, IUCF (1990).

[6] N. F. Ramsey, IEEE Trans, Instr. Meas. IM-36, 155 (1087).

[7] V. P. Chicbotayev, Appl. Phys, B 51, 303 (1990).

[8] Y. Onel, A. Penzo, and R. Rossmanith, Fundamental Symmetries, edited by P. Bloch, P. Pavlopoulos, and R. Klapisch (Plenum Publishing Corporation, 1987).

[9] M. Conte, A. Penzo, A. Pisent, and M. Pusterla, INFN Report INFN/TC-88/25.

[10] Ya. Derbenev, University of Michigan Report, UM HE 90-23; Ya. Derbenev, in proceedings of this workshop.

[11] H. Kreiser, Ph.D. thesis, Univ, of Hamburg, to be published.

[12] R. Rossmanith and R. Schmidt, Nucl. Instr. Meth. A236, 231 (1085).

(13] R. Tulman, in AIP Conference Proc, 153, New York, p. 789 (1987).

[14] Stephenson et al., IUCF Scientific and Technical Report, May 1088.

(15) B. von Przewoski et al., Proc. 9th Int. Symp, on High Energy Spin Physics, Bonn, Germany, Sept. 1990.

[16] M. G. Minty, Ph.D. thesis, Indinna University, July 1991. 
[17] John E. Goodwin, Ph.D. thesis, Indiana University, Mny 1000.

[18] W. K. Pitts et al, IUCF Scientiffe and Technichl Report, Mny 1000.

[10] F. Junes, M. Roos, Minuit, a system for function minimization und annlysis of the parameter errors and correlation, CERN/DD Internal Report 75/20. 

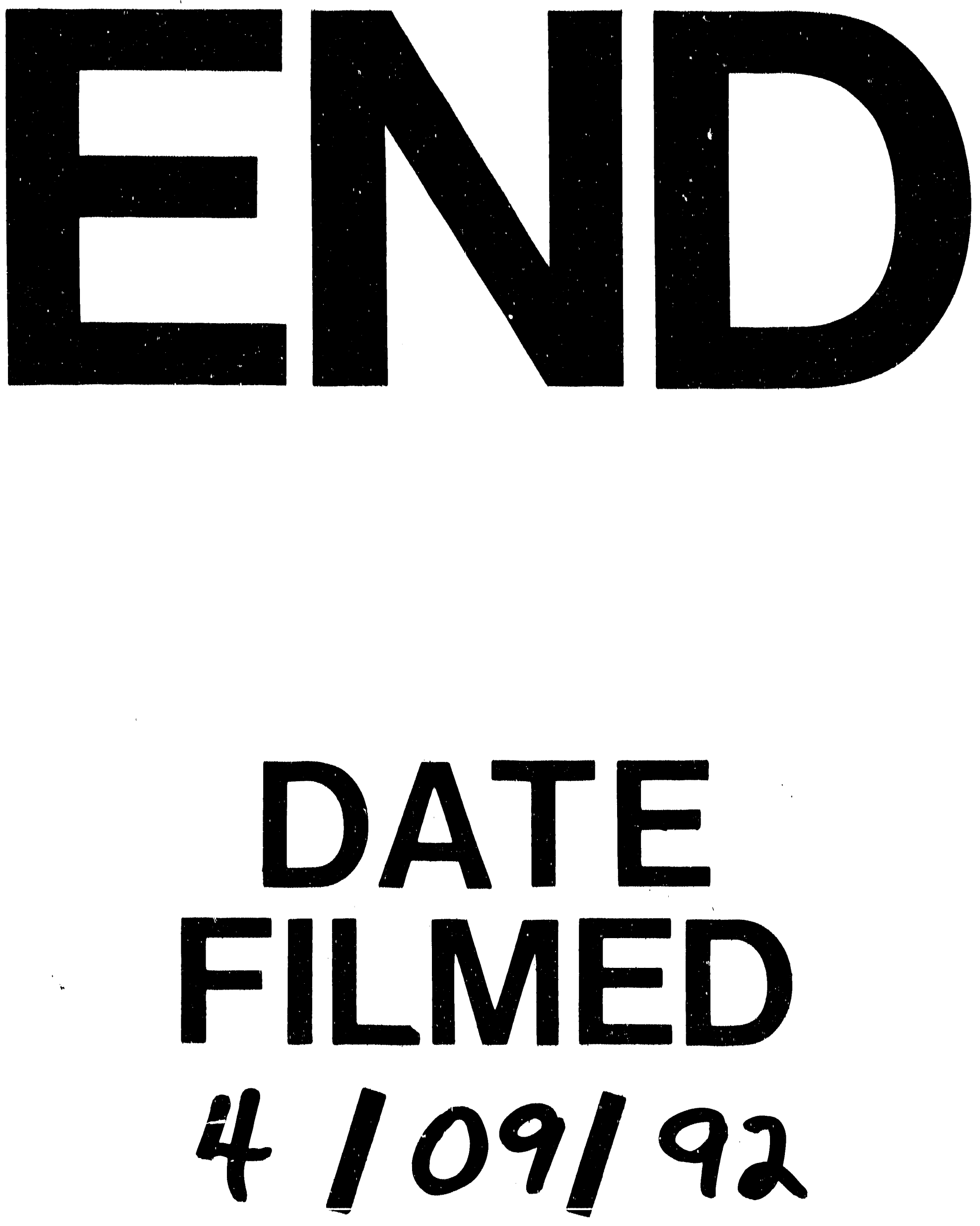

7 
